\title{
Quantum Field Thermal Machines
}

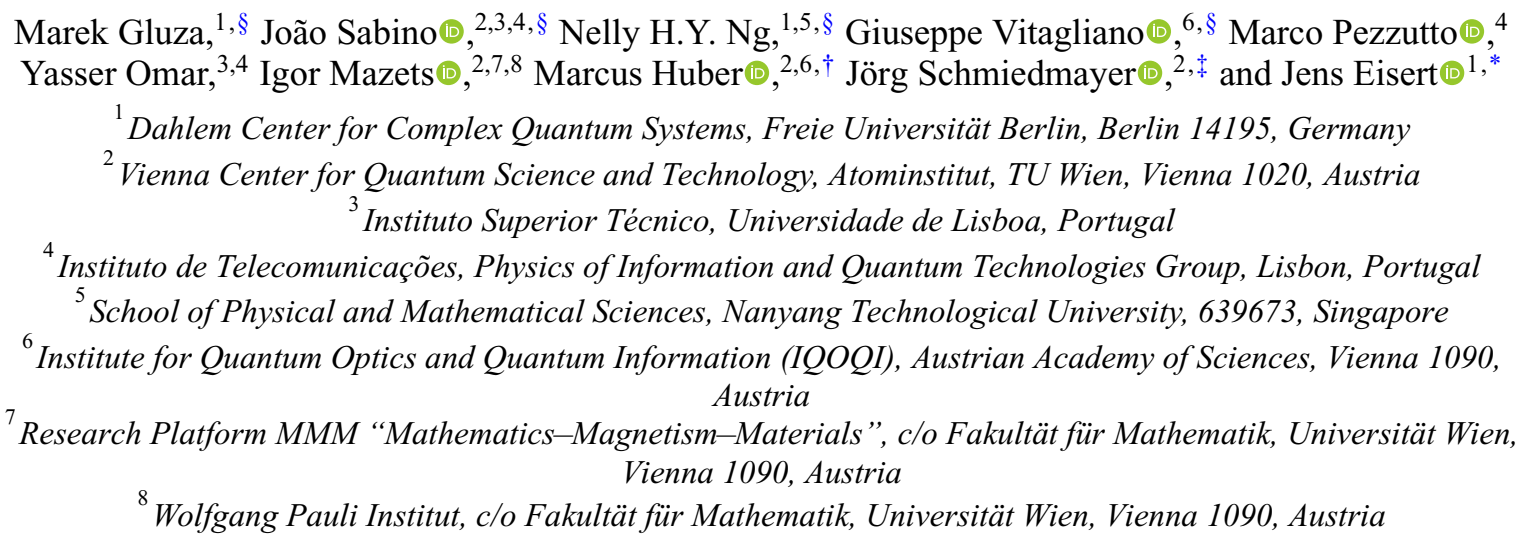

(Received 1 July 2020; accepted 16 June 2021; published 19 July 2021)

Recent years have enjoyed an overwhelming interest in quantum thermodynamics, a field of research aimed at understanding thermodynamic tasks performed in the quantum regime. Further progress, however, seems to be obstructed by the lack of experimental implementations of thermal machines in which quantum effects play a decisive role. In this work, we introduce a blueprint of quantum field machines, which — once experimentally realized — would fill this gap. Even though the concept of the QFM presented here is very general and can be implemented in any many-body quantum system that can be described by a quantum field theory. We provide here a detailed proposal of how to realize a quantum machine in onedimensional ultracold atomic gases, which consists of a set of modular operations giving rise to a piston. These can then be coupled sequentially to thermal baths, with the innovation that a quantum field takes up the role of the working fluid. In particular, we propose models for compression on the system to use it as a piston, and coupling to a bath that gives rise to a valve controlling heat flow. These models are derived within Bogoliubov theory, which allows us to study the operational primitives numerically in an efficient way. By composing the numerically modeled operational primitives we design complete quantum thermodynamic cycles that are shown to enable cooling and hence giving rise to a quantum field refrigerator. The active cooling achieved in this way can operate in regimes where existing cooling methods become ineffective. We describe the consequences of operating the machine at the quantum level and give an outlook of how this work serves as a road map to explore open questions in quantum information, quantum thermodynamic, and the study of non-Markovian quantum dynamics.

DOI: 10.1103/PRXQuantum.2.030310

\section{INTRODUCTION}

As elevated and set in stone as the basic principles of thermodynamics may appear, there is a development emerging that could not have been anticipated when this

\footnotetext{
*jense@zedat.fu-berlin.de

†'marcus.huber@univie.ac.at

\$schmiedmayerjoerg@me.com

$\S$ These authors contributed equally.
}

Published by the American Physical Society under the terms of the Creative Commons Attribution 4.0 International license. Further distribution of this work must maintain attribution to the author(s) and the published article's title, journal citation, and DOI. theory was being conceived. Indeed, the basic laws were formulated in an effort to understand the functioning of macroscopic machines that can be described by classical physics. However, due to advances in quantum technologies the question that currently begs for an answer is what happens if we consider heat engines for which quantum laws and effects are expected to play an important role. Indeed, there has been a significantly increased recent interest in exploring thermodynamic notions in the quantum regime [1-7].

One of the most notable insights that has been achieved in this context is, on the one hand, the increased role of knowledge and control giving rise to potentially superior performance of quantum machines. On the other hand, inevitable fluctuations of energy pose novel conceptual 
challenges in defining thermodynamic quantities at the quantum scale. Additionally, in the quantum regime thermal and quantum correlations may range over substantial portions of the elements of the machine, possibly influencing its dynamics. These fundamental questions have stimulated interesting experimental developments, e.g., fully controlling a quantum system such as a trapped ion [8-10], a single impurity electron spin in a silicon tunnel field-effect transistor [11] or an electronic circuit [12] to engineer behavior reminiscent of thermal machines. In ensembles of nitrogen-vacancy centers in diamond, the first quantum signatures have just been observed [13].

There is a caveat, however, constituting a serious road block in this avenue of research. It arguably turns out to be excessively difficult to experimentally realize a machine that works in the thermodynamics regime and at the same time shows genuinely quantum effects: this would be a physical system for which

(i) quantum mechanics is required to derive an appropriate effective physical model describing its dynamics, with genuine quantum correlations potentially playing a major role and

(ii) it is infeasible to control its every single degree of freedom.

Thus, ideally such a machine would consist of a quantum many-body system. The genuinely quantum behavior of such machines can in principle be witnessed by irregularities of the system going against the natural direction of entropy increase. Such irregularities are, however, generally difficult to observe due to the time scales of their occurrence being long, and therefore easily dampened by external dissipation. That this is nevertheless possible has been demonstrated in the recent observations of many-body recurrences [14,15].

Despite having the potential to play a similar role for the development of quantum thermodynamics as the steam engine did for the classical theory of thermodynamics, at the present stage, such machines have yet to be devised. This state of affairs seems a grave omission in particular in the light of the observation that it has been the study of the performance of machines that led to the development of classical thermodynamics in the first place.

In this work, we propose a blueprint for a quantum field machine (QFM) first conceived in Ref. [16] that would, once experimentally realized, qualify as being a genuine quantum thermal machine in this sense. One of the central challenges here is a trade-off between a sufficient size of the machine to meaningfully allow for thermodynamic considerations - after all, one has to make reference to thermal baths - and sufficient control of the dynamics. Only if suitable levels of control can be reached, one can hope to transcend features of classical statistical mechanics and reveal genuine quantum behavior of machines. Furthermore, elucidating quantum thermodynamic behavior will be even more important whenever the envisioned machine actually manages to perform a task that would otherwise be impossible to achieve by other means. A prominent example of such a potential task is refrigeration.

On the one hand, current cooling techniques applied to quantum systems (e.g., laser cooling, evaporative cooling) seem to have hit the ultimate (semi)classically possible limit; on the other hand, it is conceivable that quantum control over the cooling mechanism could serve to go beyond such a limit. In this sense, a genuine quantum machine could have revolutionary practical implications, very analogous to the steam engine example mentioned above. The QFM that we propose here intends precisely to address all of the aforementioned challenges posed when building genuine quantum machines:

(i) It is a genuine complex quantum many-body system, describable by means of effective quantum field theories that capture emergent degrees of freedom using different scales of refinement in the field theory model. In this particular work we focus on a QFM tuned on a Gaussian regime which is efficiently simulable numerically [17] and also a very good approximation for moderately short time scales. We, however, note that it can be implemented in a strongly correlated regime where a Gaussian treatment or even a perturbative treatment is not possible [18-20].

(ii) It offers potential new tools for quantum liquids and gases, e.g., by providing an additional stage of cooling, which does not involve diluting the system and can be applied after the use of existing techniques.

(iii) The available degrees of controllability makes it possible to exploit strong correlations and coherences for probing quantum effects. This is achieved by steering the functioning of the machine by our understanding of the physics of the system, instead of controlling individual degrees of freedom.

This anticipated device derives from ultracold atoms that in a tuneable fashion realize the full range from noninteracting to strongly correlated phononic quantum fields [18,19,21-23], as can be implemented on an atom hip [24-26]. The feature that renders it a machine is the presence of programmable time-dependent potentials allowing manipulation of the quantum fields. Such time-dependent potentials have been implemented in a one-dimensional (1D) experiment on an atom chip by means of a digital micromirror device (DMD) [27]. That is to say, the DMD devices take the role of "control knobs" of the machine, in particular also being responsible for the input of work. At the same time this field machine will operate at finite temperatures (in contrast to the majority of theoretical studies on quantum fields done with respect to the ground state), thus all these features come together when considering a QFM.

We shall start our investigation by laying out in Sec. II the concept of a QFM and describing its building blocks. In Sec. III we give a detailed introduction on how to implement a quantum field machine 
using one-dimensional quasicondensates manipulated on an atom chip with optical fields. In Sec. IV, we present a numerical study of each primitive operation described in the introduction and in Sec. V show how to compose them together to make a quantum field refrigerator and compare how it performs compared to state-of-the-art cooling techniques used in cold-atom experiments. Besides that, we discuss the phenomena of anomalous heat flow between two gases correlated by one of the primitives Finally, in Sec. VI we complete the roadmap towards building a quantum field thermal machine by highlighting the near future directions of research that we will explore.

\section{THE QUANTUM FIELD MACHINE}

Thermodynamics is a versatile framework allowing the description of a large variety of machines. Any of these ordinary thermal machines can be explored in the quantum regime if one considers operating it under conditions where quantum effects prominently play a role. This is the pathway we take in this work, by considering the working fluid to be a Bose-Einstein condensate (BEC) and in the one-dimensional regime more precisely we consider quasicondensates [28]. In order to investigate the influence of quantum effects on the machine, it is a necessity to consider an appropriate quantum model that describes the system. At the same time, it is also crucial to understand how the quantum evolution of a system can be used to implement certain abstract but well-defined thermodynamic transformations, general enough to be independent of whether quantum effects are significantly involved or not.

A quantum thermal machine can be constructed by choosing a few suitable building blocks and applying some operations on them in a cyclic fashion, forming a thermodynamic cycle. For instance, as illustrated in Fig. 1 it is instructive to consider a quantum thermal machine consisting of three elements, of which two are thermal baths, while the third is a piston shuttling between them. The relevant degrees of freedom in our machine are phonons, which we describe with an effective quantum field theory. With these ingredients it is, e.g., possible to run a heat engine, by allowing heat transfer from the hot bath to the cold one, while work can be extracted from the piston. If quantum fluctuations play a significant role, their contribution would have to be taken into account for such a process. Moreover, since the individual components of the machine are small and they feature relatively large energy fluctuations, the systems may exhibit complex out-of-equilibrium dynamics during the operation of the cycle. In this work, we demonstrate the reverse process: in particular, we operate the machine as a quantum field refrigerator, using the piston to extract heat from one part of the machine and disposing it into another part. We show that with such an active cooling mechanism it is theoretically possible to

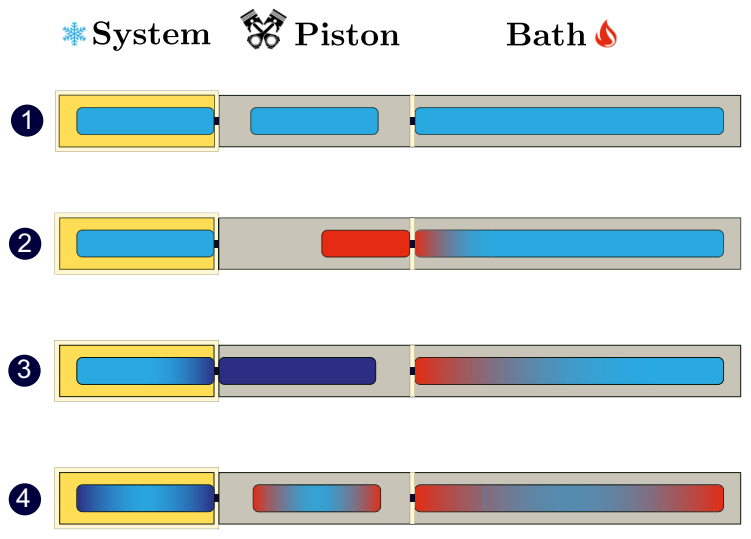

FIG. 1. Quantum field refrigerator: similar to canonical ideas employed in ordinary thermal machines, we consider for a quantum many-body system a cycle consisting of a small set of control operations on quantum working fluids, concatenated in order to cool down a part of the machine (referred to here as the "system"). This is achieved through a protocol consisting of four steps: (1) Initialization of the system, the piston and the bath at equal temperatures. (2) Compression of the piston and coupling to the bath which receives energy, and decoupling after the heat transfer. (3) Decompression of the piston, therefore decreasing its energy, then coupling to the system thus enabling heat transfer from the system to the piston. (4) Decoupling of the piston from the system and compression to initial size. Through steps (1)-(4), we expect to achieve a decrease in the system's energy, while the energy of the piston and bath should increase. This increase in energy happens in such a way that the piston and bath can be reused for multiple cycles before they saturate. All these operations can be implemented experimentally in an ultracold atomic gas, by shaping light fields that control the atoms.

cool down a system of ultracold atoms to a temperature regime in which other cooling methods are ineffective.

In order to implement such quantum field machines, we identify two basic operations, which we call quantum thermodynamic primitives (QTPs): a valve and a piston. The first allows control of the energy flow between elements of the machine. The second allows control of thermodynamic parameters during a stroke: by changing the volume, we modify pressure or temperature via the equation of state [21]. These basic ingredients of our thermodynamic protocols can be concatenated in a modular fashion to build up the complex range of potential applications for such a machine of interest. In what follows, we put particular emphasis on providing details about the functioning of a quantum field refrigerator as illustrated in Fig. 1.

\section{A. Coupling and decoupling two quasicondensates: a valve}

As depicted in Fig. 1, one of the essential ingredients for operating a quantum field machine is coupling its elements. This will be in general realized by allowing excitations to tunnel through a barrier, which controls energy flow 
between two parts, like in a valve. When considering such a valve in the quantum regime, we see some important differences compared to a similar operation in an ordinary thermal machine. Specifically, the following.

(i) In classical physics, merging of systems with identical density is largely featureless. In sharp contrast, with two quasicondensates, even if initially uncorrelated, due to phase gradients at the interface of the two systems, excitations of non-negligible magnitude are unavoidably created, and this consequently leads to an overall energy and entropy increase. Such quantum phase diffusion effects [29-32] can, however, be countered by enabling yet another quantum effect, which is coherent tunneling through a barrier, leading to phase locking [23,33-35].

(ii) Conversely, splitting two quasicondensates after they have established phase coherence may introduce quantum noise [36,37] related to the dynamical Casimir effect $[38,39]$. The production of excitations in this process, especially in a finite system would add an even larger amount of energy.

(iii) The individual elements are systems that feature correlations extending over sizeable lengths and times compared to the size and operation time scales of the machine, unlike in ordinary thermal machines. Notably, even at thermal equilibrium a single quasicondensate has a finite thermal coherence length $\lambda_{T} \neq 0[20,34]$, which would not be true if one were to simply set the reduced Planck constant to zero $\hbar \rightarrow 0$ entirely disregarding quantum effects.

(iv) Operating a valve in the quantum regime features recurrences during the evolution, an effect that has been also experimentally observed in Ref. [33], and is one of the signatures of non-Markovianity. This, among other consequences, implies that the concatenation of cycles of the QFM depends on the very precise timing of the individual elementary operations (i.e., the QTPs).

\section{B. Compressing and decompressing: a piston}

The defining feature of a piston is that its size can be changed, which, via the equation of state [21,40,41], leads to a change of internal energy. Because of this, the main role of the piston is that even if all the parts of the quantum field machine are in thermal equilibrium, one can introduce temperature differences by performing work upon the piston. This, in combination with the valve, enables heat flow in the desired direction. Again, if the physics of the piston involves quantum effects one can expect certain differences to ordinary thermal machines. For example, the following.

(i) While the energy is changing due to compression or decompression the piston may go out of thermal equilibrium, e.g., due to squeezing of internal modes [17,39].

(ii) Internal dynamics in the QFM elements occur within time scales comparable to timings of individual steps of the cycles considered. In contrast, in classical thermal machines concrete time scales are not comparable and hence usually discarded.

(iii) The piston essentially consists of a moving boundary, which is closely related to the dynamical Casimir effect [39].

To conclude this section, let us emphasize that these effects are particularly relevant also for practical applications. For example, while the amount of energy injected in a local operation is intensive, its effects are substantial. All of these effects jointly influence the quantitative performance of the quantum field machine, as we also observe in the numerical study that follows. In particular, regarding a general discussion about the efficiency of a quantum field machine see also Sec. VIB.

\section{IMPLEMENTING QUANTUM FIELD MACHINES IN 1D BOSE-EINSTEIN QUASICONDENSATES}

This section discusses the basics for implementing a quantum field machine on ultracold one-dimensional gases. In Sec. III A we describe the microscopic model and the related effective Hamiltonian defining the energy of phononic fields. Section III B describes concisely the role of the DMD in engineering the desired QTPs, closely matching the experimental state of the art [27]. Finally, we discuss various diagnostic methods in Sec. III C.

\section{A. Effective quantum field theory description of 1D cold atoms}

Cold atomic gases at low temperatures and with a fixed average number of atoms are effectively one dimensional if the trap anisotropies are sufficiently large to constrain the dynamics in two (transversal) dimensions such that the dynamics effectively takes place in the remaining (longitudinal) direction [19,28]. In this regime, the system is well described by the Lieb-Liniger Hamiltonian, which reads

$$
\hat{H}_{\mathrm{LL}}=\int d z \hat{\Psi}^{\dagger}\left[\frac{-\hbar^{2}}{2 m} \partial_{z}^{2}+V(z, t)-\mu+\frac{g}{2} \hat{\Psi}^{\dagger} \hat{\Psi}\right] \hat{\Psi} .
$$

Here $\hat{\Psi}(z)$ is the atomic annihilation operator at spatial position $z$, which satisfies bosonic exchange statistics $\left[\hat{\Psi}(z), \hat{\Psi}^{\dagger}\left(z^{\prime}\right)\right]=\delta\left(z-z^{\prime}\right)$. The atomic mass is denoted by $m$ and $\hbar$ is the reduced Planck constant. The external potential $V(z, t)$ is responsible for longitudinal trapping of the gas but can be also used as a means of implementing the necessary control operations for the machine. The quartic interaction has strength $g / 2$, which is proportional to the scattering length of the atoms, and also depends on other characteristics of the trap, specific of the experimental implementation [33]. Finally, $\mu$ is the chemical potential that can be fixed, e.g., by constraining the average 
number of atoms $N_{\text {atoms. }}$. In a semiclassical theory of such gases, the study of its evolution is constrained to the set of coherent states, thereby approximating the field operators by a classical wave function, that obeys the so-called Gross-Pitaevskii (GP) equation.

The variational ground-state atomic density calculated from the GP equation, which we denote as $\rho_{0}(z)$, has the interpretation of the mean-density profile that can be measured by in situ density absorption [27,34] [see Eq. (A1), Appendix A]. By expressing the field operators in the polar decomposition,

$$
\hat{\Psi}(z)=\sqrt{\rho_{0}(z) \hat{\mathbb{1}}+\delta \hat{\varrho}(z)} e^{i \hat{\varphi}(z)},
$$

the GP equation translates into a system of hydrodynamic equations of a superfluid in the density-phase variables [42]

$$
\begin{aligned}
\partial_{t} \rho_{0}+\partial_{z}\left(\rho_{0} v\right) & =0, \\
\partial_{t} v+v \partial_{z} v & =-\frac{1}{m} \partial_{z}\left(V-\mu+\frac{1}{\rho_{0}} P+Q\right),
\end{aligned}
$$

where we define $v(z, t)=\hbar \partial_{z} \varphi(z, t) / m$ as the fluid velocity, and the terms $P$ and $Q$ are referred to as the pressure and quantum pressure term, respectively,

$$
P=g \rho_{0}^{2} / 2, \quad Q=-\frac{\hbar^{2}}{2 m \sqrt{\rho_{0}}} \partial_{z}^{2} \sqrt{\rho_{0}} .
$$

Neglecting the last term $Q$, one obtains a set of Euler equations describing the flow of a nonviscous fluid with equation of state $P\left(\rho_{0}\right)=g \rho_{0}^{2} / 2$. This is a semiclassical approximation to our system. In this approximation the gas has, at zero temperature, energy density $e\left(\rho_{0}\right)=g \rho_{0}^{2} / 2$ and chemical potential $\mu\left(\rho_{0}\right)=g \rho_{0}$. Such an approximation is nevertheless insufficient to capture all quantum effects we aim at studying. Therefore, we employ a fully quantum treatment of the (linearized) evolution.

For inhomogeneous systems, namely $\rho_{0}(z) \neq$ const, the model cannot be solved exactly due to the quartic term. However, it is well known that a quadratic approximation in the spirit of the Bogoliubov theory captures low-energy excitations $[18,21]$ and works very well for certain time scales [17]. The effective model is obtained by expanding the Hamiltonian up to second order in the density $\delta \hat{\varrho}(z)$ and phase $\hat{\varphi}(z)$ fluctuation operators, which are again bosonic $\left[\delta \hat{\varrho}(z), \hat{\varphi}\left(z^{\prime}\right)\right]=i \delta\left(z-z^{\prime}\right) \hat{\mathbb{1}}$. They represent phononic excitations of a cold atomic gas and their energy is given by the following effective phononic Hamiltonian

$$
\hat{H}_{P}\left[\rho_{0}\right]=\int d z\left\{\frac{\hbar^{2} \rho_{0}(z)}{2 m}\left[\partial_{z} \hat{\varphi}(z)\right]^{2}+\frac{g}{2} \delta \hat{\varrho}^{2}(z)\right\},
$$

which can be decoupled in normal phononic modes. An important feature of this model is that wave packets travel with a speed of sound related to the mean density $c=\sqrt{g \rho_{0} / m}$.

The model in Eq. (5) provides a good effective description for experiments performed on an isolated quasicondensate [20,33,43-45]. However, in our simulations the QFM couples its initially isolated elements. Then, one has to additionally model what happens with the phase zero modes in the systems. A phase zero mode has the interpretation of the total momentum frame of the excitations. For an isolated system, this mode allows for phase fluctuations without an energy cost [29-32]. However, when two thermal systems, each with their individual zero mode, are coupled, the two zero modes hybridize to form the joint zero mode and one mode with fluctuations that cost energy. The energy cost can be large if the original phase zero modes are nontrivially populated, since the phase difference of two independent systems is fully random. Nevertheless, this is different in the physical system where the energy changes continuously. This can be described when considering a more refined modeling using the full Hamiltonian (1), which would dynamically induce phase locking between the two condensates during the process. Via the large coupling expansion of $\hat{H}_{\mathrm{LL}}$, or arguing phenomenologically, an effective model can be derived that reads

$$
\hat{H}\left[\rho_{0}\right]=\hat{H}_{\mathrm{P}}\left[\rho_{0}\right]+2 \pi \hbar \int d z J(z) \rho_{0}(z) \hat{\varphi}^{2}(z),
$$

where the additional term regularizes the zero modes. In our main simulations, we make the modeling simplification $J=$ const, effectively gapping out the phase zero modes across the condensate at all times. The presence of this additional term can be interpreted as the quasicondensates being merged having been already phase locked prior to the merging. The phase-locking term effectively induces squeezing of the modes, which can be analytically seen in the homogeneous case. Quantitatively, in the numerical study that follows we use a small value $J=20 \mathrm{mHz}$. Meanwhile, Appendix C3 contains a further discussion on using a more generic $J(z)$.

\section{B. Controlling the 1D quantum field simulator using a DMD}

To achieve the QTPs described in Sec. II, the longitudinal trapping potential $V(z, t)$ has to be precisely manipulated. For that, it is possible to create a dipole trap (which adds to the magnetic chip trap) by shining blue-detuned light on the atoms, which creates a conservative repulsive potential [46]. By spatially manipulating this light, one would be able to nearly arbitrarily shape the trap or add features to the existing magnetic trap.

Using a device such as the DMD for this purpose is a standard technique for many cold-atom experiments [47-51] (see also Ref. [52] for a review). In our specific 
platform, we use a device with $1920 \times 1080$ (full HD) micromirrors that can be turned on (sending light to the atoms) or off (sending light outside of the optical path). The whole two-dimensional (2D) array of mirrors spans a spatial region, which is approximately 10 times the size of the BEC and each mirror in the DMD contributes with a Gaussian distribution of light, with a width of $0.4 \mu \mathrm{m}$ in the plane of the atoms. This is roughly twice the healing length and several orders of magnitude smaller than the phase coherence length. Moreover, the fact that the DMD used in our platform has a refresh rate of $32 \mu \mathrm{s}$ ( 3 orders of magnitude faster than the time scale of the atoms), allows the 1D potentials to effectively vary continuously in time. In fact, in Ref. [27], it has been demonstrated that different 1D potential landscapes can be implemented with a very high degree of control in this experimental setup.

It is also worth stressing that optimal control techniques can be used for the realization of the valve and piston QTPs in the experiment in a way maximizing the stability of the system. In Refs. [53,54], it has been demonstrated that, for the case of compressing the gas in a harmonic trap, it is possible to find shortcuts to adiabacity. In this case, a single control parameter has been suitably optimized, which has been the frequency of the longitudinal harmonic trapping potential. This has allowed expansion of the gas without introducing longitudinal breathing of the mean density, which hints that optimal control should also be important for implementing a piston using a DMD potential. Similarly, for the valve it is important to switch on the coupling between the two systems, without introducing stray excitations into the system, which again can be optimized by appropriately tailored time-dependent potentials using the DMD. Performing optimal control of the elements of the QFM will have to take into account that a very fast manipulation of the cold atomic gas can enter into a supersonic regime, which leads to exciting physical effects that have been explored experimentally for the expansion of the gas [50], which is important for the piston and for local manipulation of the gas [55-57], which is relevant for the valve.

\section{Space-and-time-resolved monitoring of thermodynamic transformations}

In order to monitor the operation of a quantum thermal machine, observables that reveal local and global information about the state of the system are needed. Of special interest are for example atomic density, spectrum and occupation of excitations, or their coherences and correlations. These physical observables allow the monitoring and understanding of the details of thermodynamic processes, such as heat or entropy flow during the operations and the global thermodynamic properties for the qualitative analysis.
There are several well-established methods to probe 1D quantum systems. These range from in situ measurements of density fluctuations [58-62] to measuring phase fluctuations in time of flight by either "density ripples" $[63,64]$ or interference $[65,66]$. Information is extracted by analyzing the full distribution functions [67] or correlation functions $[20,34,43,44]$. It will be crucial to use these measurement methods to extract information about local properties of the system. This detects the action of local control when implementing the envisioned operations and resolving the thermodynamic transformations occurring in the elements of the QFM. Of specific interest, when probing the quantum thermodynamic processes, is the (local) occupations of excitations of the quantum fields, i.e., of the phonons. We first observe that the energy of the phonons in the system is defined as the expectation value of the quadratic Hamiltonian (5). Note that the coupling coefficient in the additional term in Eq. (6) is chosen precisely such that its overall contribution to the energy is negligible and it renders negligible also the contribution of the zero modes while merging two systems. Thus, by integrating Eq. (5) over the length of the condensate one would obtain the total energy of the system. On the other hand, access to the local phase-phase fluctuations

$$
C^{\phi \phi}\left(z, z^{\prime}\right)=\left\langle\hat{\varphi}(z) \hat{\varphi}\left(z^{\prime}\right)\right\rangle
$$

and to the second moments of local density fluctuations

$$
C^{\rho \rho}\left(z, z^{\prime}\right)=\left\langle\delta \hat{\varrho}(z) \delta \hat{\varrho}\left(z^{\prime}\right)\right\rangle
$$

also directly implies the knowledge of the local energy density, which is given by

$$
\frac{d E(z)}{d z}=\left.\frac{\hbar^{2} \rho_{0}(z)}{2 m} \partial_{z_{1}} \partial_{z_{2}} C^{\phi \phi}\right|_{z_{1}=z_{2}=z}+\frac{g}{2} C^{\rho \rho}(z, z) .
$$

Note that the cross-correlations between phase and density degrees of freedom

$$
C^{\phi \rho}\left(z, z^{\prime}\right)=\left\langle\hat{\varphi}(z) \delta \hat{\varrho}\left(z^{\prime}\right)\right\rangle
$$

do not contribute to energy and vanish in thermal equilibrium, though may be nonzero during out-of-equilibrium dynamics. At this point, two comments are in order.

(i) The expression of the local energy, Eq. (9), needs to be regularized due to divergences at the point $z_{1}=z_{2}$. This is accounted for by considering a UV cutoff in the corresponding field theory, in order for the energy in the system to be finite.

(ii) The UV cutoff emerges naturally in the experiment. This is due to its finite imaging resolution and effects of "smearing" in time of flight [66]; therefore, one can measure only a coarse-grained expectation value of the fields averaged over a finite length scale $\sigma_{\text {res }}$, and higher momentum modes cannot be detected. 
The gradient of the phase operator $\hat{v}=\partial_{z} \hat{\varphi}$ can be interpreted as the velocity of wave packets traveling on top of the condensate (as per hydrodynamic description). Thus, the first term in the Hamiltonian (5) can be thought of as the energy content related to the speed of wave packets, while the other term to how much distortion to the local density they induce. It is important to note that both contributions must be measured in order to have the complete information about the energy in the system. As mentioned earlier, on the atom-chip platform, it is possible to measure experimentally by observing the quasicondensate in situ transversely (from the side) by means of density absorption [58-62]. In Appendix A, we discuss how measurements of the local density fluctuations of the atomic gas gives access to direct measurement of the GP profile $\rho_{0}$ and the second moments of the density fluctuations $\Gamma^{\rho \rho}$. Additionally, in Appendix A we describe a proposal for a tomographic reconstruction method similar to Ref. [17]; based on outof-equilibrium data of $\Gamma^{\rho \rho}(t)$ at different times $t$, one can recover $\Gamma^{\phi \phi}$. This then provides access to the second moments of phase fluctuations and hence the energy in the phase sector can be extracted.

Alternatively, one can envision interfering the system under study with a local oscillator [a large threedimensional (3D) BEC] [47] or with an identical system $[43,44,66]$ to extract the local phase correlations $C^{\phi \phi}$. From them one can tomographically reconstruct correlations of density fluctuations $C^{\rho \rho}$ [17]. If one can assume thermal equilibrium, then it is possible to extract the occupation numbers of phonons even from $C^{\phi \phi}$ alone [44]. Global parameters like temperature can then be obtained also by "density ripples" $[63,64]$. The temperature is typically extracted by means of an appropriate fit to the correlations of the fluctuations of the atoms after a time-of-flight expansion.

It is important to understand which thermodynamic transformations have a substantial effect that is clearly detectable in the experiment. The precision for measuring the (changes) in temperature or energy in the system will depend on the reliability of the state preparation and the statistical sample size. We anticipate that changes of temperature or energy by about $10 \%$ should be large enough to obtain conclusive experimental results [33] $(>5 \sigma)$ where one can be confident about, e.g., observing heat flow or cooling in a given system.

\section{NUMERICAL STUDIES OF QUANTUM THERMODYNAMIC PRIMITIVES}

As sketched in Fig. 1 above, the piston and the valve are building blocks that allow construction of a refrigeration cycle. In this section, we present results on the numerical modeling of the individual quantum thermodynamic primitives involved.
Each QTP that we propose is modeled by a Hamiltonian of the form Eq. (6) described in the previous section, which allows us to simulate the dynamics of phonons and to calculate corresponding energy changes in the system. As the model is quadratic, our simulations are done within the Gaussian framework and are computationally efficient. Moreover, this description allows us to efficiently evaluate information-theoretic entropies of such systems (e.g., relative entropy), which are relevant for thermodynamics of finite-sized quantum systems.

Our model allows us to derive core predictions in the framework described in Sec. III A. In our simulations we use parameters that fit state-of-the-art experiments of 1D quasicondensates performed on the atom-chip platform. More generally, our proposal is embedded in the broader framework of thermodynamics with multimode Gaussian states, with Gaussian operations modeling the action of external system control.

\section{A. Coupling and decoupling two quasicondensates: a valve}

Adjusting the external potential makes it possible to split the gas into two parts or merge at will [36]. We then study energy and correlation changes during the merging process. A simple model is considered, where two quasicondensates are coupled via a small buffer region. Specifically, we consider a bipartite system, with each part $A$ and $B$ initially thermal and approximately homogeneous, the two parts being separated by a buffer region of negligible size $\ell \sim \xi_{h}$ so that phonons cannot tunnel. The Hamiltonian in Eq. (6) is specified by the GP profile, which we choose with a shape according to Fig. 2(a). Lastly, we specify Neumann boundary conditions (NBCs) at the edges. The density profiles that we choose have precisely the scope of smoothening further the boundary conditions in our implementation via a discretized lattice model.

Denoting $\rho_{0}^{A}$ and $\rho_{0}^{B}$ as GP profiles of parts $A$ and $B$, respectively, the initial Hamiltonian of the full system reads

$$
\hat{H}_{A \mid B}=\hat{H}\left[\rho_{0}^{A}\right]+\hat{H}\left[\rho_{0}^{B}\right],
$$

where the tiny separation at the interface is modeled by the Hamiltonian $\hat{H}_{A \mid B}$ having in total four NBCs, two at the edges and two in the middle. Next, we define the joint system to have a GP profile

$$
\rho_{0}^{A B}(z)= \begin{cases}\rho_{0}^{A}(z) & z \in A, \\ \rho_{0}^{B}(z) & z \in B,\end{cases}
$$

implementing the "gluing" of the profiles. Thus, in our minimal modeling approach, we neglect the precise spatial details of experimental control necessary to switch from two independent systems to the coupled case as we assume 
(a)

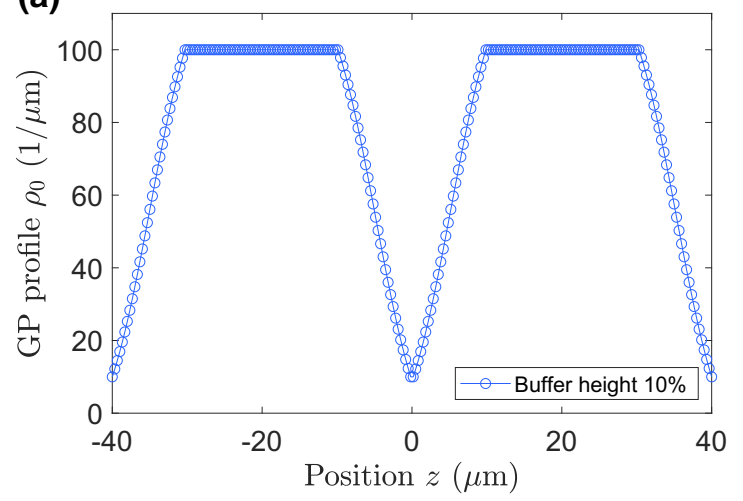

(b)

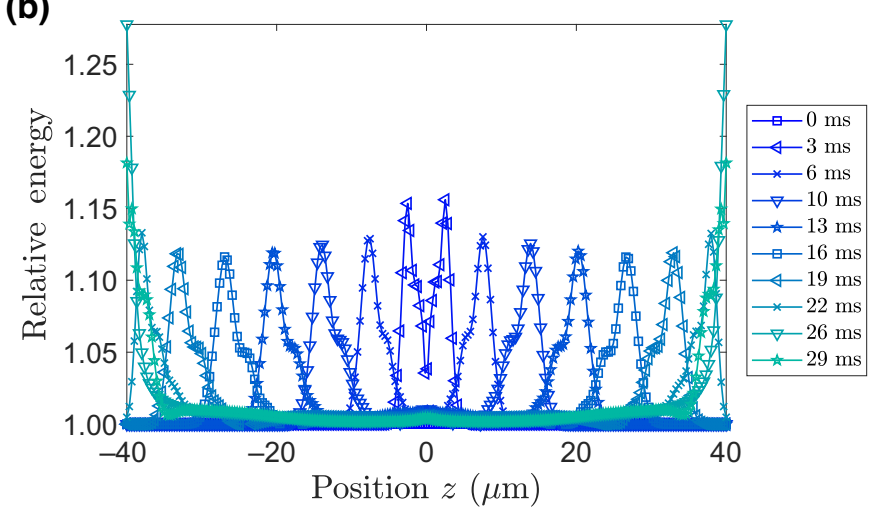

FIG. 2. Operating a valve between two identical and independent thermal quasicondensates. (a) GP profiles. We consider two quasicondensates, which are homogeneous in the bulk but their density falls off towards zero at their edges. At position $z=0$ there is initially the boundary condition that in our effective model at a single point implements the separation between the two systems. As the systems become coupled the energy can tunnel between the two systems through this point. Throughout on-line plots of real-space quantities bullets indicate the discretization lattice used in the simulation while the continuous lines are merely a guide to the eye. (b) Dynamics of energy density. We plot $d E(z) / d z$ defined in Eq. (9) for different times during the coupling of two quasicondensates. Initially, the energy density in each quasicondensate is uniform, and we use that value to normalize the plotted values. During the coupling, localized energy is injected at the interface of the two systems and travels ballistically away in the form of wave packets, which increase the energy density by approximately equal to $15 \%$.

that they are close and only a microscopic change is necessary for removing the small buffer region. With that, we can take the final Hamiltonian of the merged systems to be

$$
\hat{H}_{A B}=\hat{H}\left[\rho_{0}^{A B}\right]=\hat{H}_{A \mid B}+\hat{H}_{\text {int }}
$$

This joint Hamiltonian has only two NBCs, and there is an interaction $\hat{H}_{\text {int }}$ between $A$ and $B$. Due to this coupling, the thermal state of $\hat{H}_{A B}$, in contrast with that of $\hat{H}_{A \mid B}$, contains correlations between $A$ and $B$.

Note that during this evolution the boundary conditions at the interface change dynamically. We handle this boundary condition issue by interpolating linearly between the uncoupled Hamiltonian with four NBCs and the coupled Hamiltonian with two NBCs. Thus, we model the time-resolved dynamics of the merging protocol by the time-dependent Hamiltonian

$$
\hat{H}_{A-B}(t)=\left(1-\frac{t}{t_{\text {merge }}}\right) \hat{H}_{A \mid B}+\frac{t}{t_{\text {merge }}} \hat{H}_{A B},
$$

within $t \in\left[0, t_{\text {merge }}\right]$. Here we model the situation that the change in the external potential makes the density profiles become smoothly interpolated. Note also that we perform a lattice discretization to compute the physical quantities of interest (see Appendix C) and in this framework mixing boundary conditions is well defined.

Subsequently, we consider two independent thermal quasicondensates, and adapt initial conditions that are natural for experiments, where evaporative cooling yields a thermal distribution of phonons with temperatures $T_{A}=$ $T_{B}=50 \mathrm{nK}$ at initial time $t=0$. Thermal states are defined with respect to a given Hamiltonian $\hat{H}$, and the density matrix reads

$$
\hat{\gamma}_{T}[\hat{H}]:=\mathcal{Z}^{-1} e^{-\hat{H} /\left(k_{B} T\right)},
$$

where $\mathcal{Z}=\operatorname{Tr}\left(e^{-\hat{H} /\left(k_{B} T\right)}\right)$ is the partition function and $k_{B}$ is the Boltzmann constant. We use GP profiles with peak density $\rho_{0}^{A}=\rho_{0}^{B}=100$ atoms $/ \mu \mathrm{m}$, smoothly falling off towards smaller values at the edges, see Fig. 2(a). These choices reflect typical experiments realized in a box trap of size $L=50 \mu \mathrm{m}$ with $N_{\text {atoms }}=5000$. The falloff at the edges according to the erf function has been chosen phenomenologically - any trap that is not infinitely strong will lead to a smooth falloff at the edges.

In Fig. 2(b) we show numerical results for a linear ramp with merging time $t_{\text {merge }}=40 \mathrm{~ms}$. This is a relatively long time scale, chosen to demonstrate that excitations can be reflected at the edge and start returning towards the interface. Initially, energy is distributed homogeneously in $A$ and $B$ so we present the energy distribution relative to that value. This relative measure is employed throughout, since it allows us to disregard the cutoff-dependent shift coming from zero-point fluctuations. In fact, our effective Hamiltonian is not normal ordered but instead regularized by the healing length $\xi_{h}=\hbar /(m c)$ of the system (note that the cutoff $\Delta z$ in our numerical simulations is smaller than the healing length). As anticipated, merging two systems via tunnel coupling induces excitations in form of counterpropagating wave packets, see Ref. [55] for a detailed experimental and theoretical study of the dynamics of such excitations. The wave packets travel with the respective speed of sound, which in typical experiments on the 
atom-chip platform is $c \approx 2 \mu \mathrm{m} / \mathrm{ms}$ [33]. The simulation predicts that the wave packets increase the local energy by a sizeable amount of approximately $15 \%$. This may cause system dynamics to deviate from the linearized approximation. Nevertheless, the higher-order terms should have only the effect of dispersing the wave packets. According to our simulations, the amount of injected excitations is higher if systems are coupled at peak density, see Appendix C2. This is because in the lattice approximation we are adding an off-diagonal coupling between the two edges of $A$ and $B$ that scales $\propto \rho_{0}(z=0)$ with density. Therefore, merging is "softer" if it occurs at a lower density value. Physically speaking, it is more stable to couple two sensitive systems harboring gapless excitations through diluted regions compared to at peak density.

It is instructive to analyze the correlations of the coupled state during the merging. As shown in Fig. 3, we find that initially there are no correlations between $A$ and $B$ and hence we see that two independent thermal quasicondensates are not thermal with respect to the joint Hamiltonian.

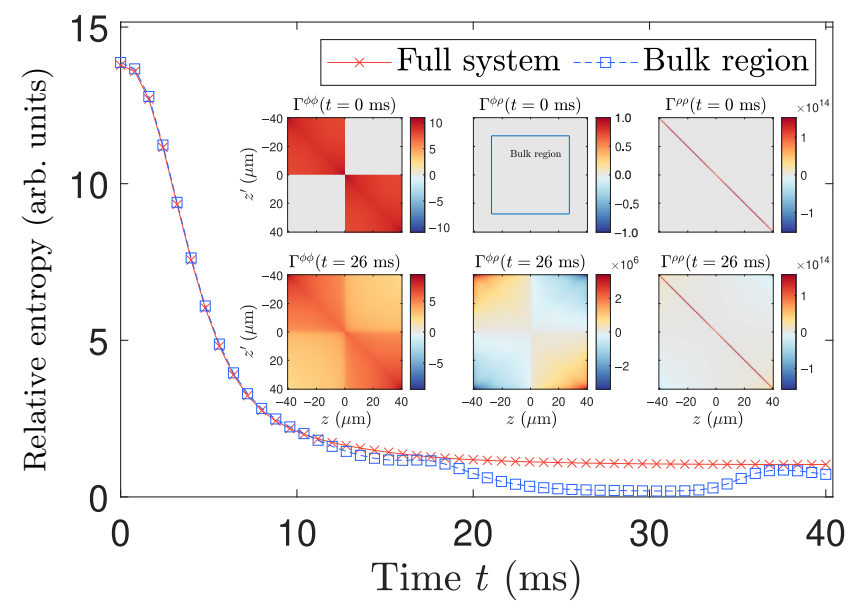

FIG. 3. Correlations before and after merging. The initial covariance matrix $\Gamma(t=0 \mathrm{~ms})$ (inset top) is characterized by phase fluctuations $\Gamma^{\phi \phi}=2 C^{\phi \phi}$ ranging only over the individual systems, no cross-correlations between phase and density operators $\Gamma^{\phi \rho} \equiv 0$, and density fluctuations $\Gamma^{\rho \rho}=2 C^{\rho \rho}$ being essentially diagonal. When heat excitations reach the edges, the covariance matrix $\Gamma(t=26 \mathrm{~ms})$ (inset bottom) restricted to the bulk region of the system agrees with the thermal covariance matrix of the joint Hamiltonian: phase fluctuations $\Gamma^{\phi \phi}$ become uniform over the joint system, in the bulk of the system crosscorrelations vanish $\Gamma^{\phi \rho} \approx 0$, while density fluctuations $\Gamma^{\rho \rho}$ are diagonal. Quantitatively, we plot the relative entropy of the timedependent covariance matrix with respect to that of the thermal state of the coupled Hamiltonian at $T=50 \mathrm{nK}$ and observe that it decreases rapidly over around $10 \mathrm{~ms}$. Due to the presence of the heat wave packets, the relative entropy for the full system (red crosses) does not converge to zero over time, while for the covariance matrix restricted to the bulk region (blue squares) it essentially vanishes at around $t=26 \mathrm{~ms}$ and then increases again.
During merging, the parts become coupled and the established correlations drive the state towards being close to the joint thermal state, see Appendix C2 for more details. Interestingly, after the first traversal time, i.e., when a local excitation at the merging interface has traveled to the edges, the joint system is already close to being thermal in the bulk (cf. inset of Fig. 3).

The observation that the merged parts become jointly thermal can be further quantified by evaluating the relative entropy, given for any two states by $S(\hat{\gamma} \| \hat{\sigma})=$ $\operatorname{Tr}[\hat{\gamma}(\log \hat{\gamma}-\log \hat{\sigma})]$. Evaluating this with respect to a thermal state yields

$$
S\left(\hat{\gamma} \| \hat{\gamma}_{T}[\hat{H}]\right):=\left(F(\hat{\gamma})-F\left(\hat{\gamma}_{T}[\hat{H}]\right)\right) /\left(k_{B} T\right) \geq 0
$$

where $F(\hat{\sigma})=\operatorname{Tr}(\hat{H} \hat{\sigma})-k_{B} T S(\hat{\sigma})$ is the free energy of the state relative to the ambient temperature $T$ and the Hamiltonian $\hat{H}$. Here $S(\hat{\varrho})=-\operatorname{Tr}(\hat{\varrho} \log \hat{\varrho})$ is the von Neumann entropy. Notably, the relative entropy is zero if and only if the two covariance matrices are the same (see Appendix B for further details). This makes it a strong measure of deviation from thermal equilibrium. Finally, this measure can be computed also for reduced density matrices, which then captures how systems are similar locally.

In order to check if the merging QTP is intensive we calculate the relative entropy of the state evolving during merging with respect to the thermal state of the coupled Hamiltonian at $T=50 \mathrm{nK}$. Initially, the relative entropy decreases rapidly, reflecting the ongoing thermalization around the interface of the two systems, where the correlations are being established. For the whole system the relative entropy does not reach zero and levels off to a constant value within about $10 \mathrm{~ms}$. This is due to the wave packets being always present in the system, hence the impossibility for the entire system to be in thermal equilibrium. If we consider the reduced covariance matrix describing only the bulk middle region, we see that around $20 \mathrm{~ms}$ the relative entropy drops essentially to zero. This means that once the excitations leave the window of observation, the system left behind agrees in that region with the (joint) thermal state. Finally, for longer times the wave packets come back to the bulk and allow for detecting an out-of-equilibrium component of the state.

We expect that features observed in this numerical study should remain true even under perturbations to the model and thus that temperature for locally merged systems is an intensive generic feature of this QTP. This is because perturbations are not expected to change the character of low-energy excitations so the spectrum should remain approximately linear and a local change of the Hamiltonian should generically create a localized surplus of energy propagating through the system with the speed of sound.

In the case presented here, we have shown an example where there has been no net heat flow between two 
systems. The next section shows how to enable heat flow between two systems, by performing work from outside, thereby creating an effective temperature difference. As an outlook, in Sec. VIA we also present the case of two initially different temperatures, observing that nonMarkovianity effects in this case are even more pronounced and can potentially lead to further interesting effects, such as anomalous heat flow.

\section{B. Compressing and decompressing: a piston}

In this subsection, we see how external control, which compresses or expands the gas, enables a condensate to function as a piston. The external control will effectively perform work on the quasicondensate, increasing or decreasing its energy depending on the change in volume. This is similar to thermodynamics of an ideal gas with the difference that we are considering a quantum manybody system. Experimentally, operations for this QTP have already been implemented with use of shortcuts to adiabacity (see Ref. [53] where the extension of the GP profile has been stably modified).

Here, we propose a model to describe what happens to phonons when the confining trap (space occupied by the gas) changes. Let the length of a uniform system change continuously over time in the sense that a homogeneous GP profile $\rho_{0}$ with support of length $L$ changes to $\rho_{0}(t)$ with corresponding length $L(t)$. The operation is assumed to preserve the atom number $N_{\text {atoms }}=\rho_{0} L$ so that

$$
\rho_{0}(t)=\rho_{0} \frac{L(0)}{L(t)}
$$

This time-dependent GP profile assumes that the change in volume is slow so that a homogeneous system remains homogeneous at all times. Under this assumption, the Hamiltonian (6) parametrized by a time-dependent GP profile $\rho_{0}(t)$

$$
\hat{H}(t)=\hat{H}\left[\rho_{0}(t)\right]
$$

describes the phonons during the size change. Using Eq. (18), the integration in Eq. (6) ranges over the timedependent length of the system $L(t)$. In the lattice approximation this is implemented by discretizing the Hamiltonian at each time considered, and identifying the respective cells at consecutive times as they change only infinitesimally. It is also possible to consider formulating the procedure using a fixed representation of momentum mode and time-dependent eigenmode wave functions [39].

In the homogeneous case by a change of the integration variable we can write the time-dependent Hamiltonian as

$$
\hat{H}(t)=\int_{0}^{L(0)} d z\left[\frac{\hbar^{2} \lambda^{2}(t) \rho_{0}}{2 m}\left(\partial_{z} \hat{\varphi}\right)^{2}+\frac{g}{2} \lambda(t) \delta \hat{v}^{2}+h J \rho_{0} \hat{\varphi}^{2}\right],
$$

where we also define a rescaled density fluctuation field $\delta \hat{v}=\delta \hat{\varrho} / \lambda(t)$ in order to preserve the canonical commutation relations. In other words, this way we have $\left[\delta \hat{v}(z), \hat{\varphi}\left(z^{\prime}\right)\right]=i \delta\left(z-z^{\prime}\right)$. Here we make the integration limits explicit and change the frame so that the length of the system is effectively constant but the Hamiltonian density becomes time dependent due to the dimensionless ratio

$$
\lambda(t)=\frac{L(0)}{L(t)}
$$

We observe that if the system stays homogeneous, then the time-dependent Hamiltonian (19) has the same momentum eigenmodes at all times $t$, but they become squeezed. We should hence expect that compressing introduces squeezing of phase and density quadratures. See Appendix C5 for an extended discussion, including the numerical implementation of the compression model and see also Ref. [39] for a related study.

With this model we can simulate the functioning of a piston: in Fig. 4 we show the results of a simulation of a single stroke. It is moreover possible to check whether the piston remains thermal during the process. We first observe that the energy density stays homogeneously distributed at all times [cf. Fig. 4(a)]. Moreover, it changes in relation to volume: as shown in Fig. 4(b), the total energy increases and comes back to the initial value during the stroke of the piston. Nevertheless, a more refined check involving the relative entropy shows that the system is not at thermal equilibrium at all times. In particular, at a sequence of times during the evolution we evaluate the relative entropy between the time-dependent state and the thermal states corresponding to the system Hamiltonian. The thermal state with the lowest relative entropy gives then the effective fit for the temperature. It is clear that if the timedependent state remains thermal at all times, then there will be a temperature for which the relative entropy vanishes. However, we find that this value is strictly positive, which indicates that the piston is away from thermal equilibrium during the compression-decompression process, and returns to thermal equilibrium only when reaching its original length. This effect can be naturally explained by the presence of squeezing in the system, but we focus here on the thermodynamic aspects of the model and refer to Ref. [39] for a discussion of the dynamical Casimir effect.

We can now use the compression QTP in order to enable heat flow between two systems. In Fig. 5, we show the steps (1)-(2) of the Otto cycle that are sketched in Fig. 1, i.e., we compress the piston, couple it to the bath and after decoupling decompress it back to its initial state. As before, piston and bath are initially both thermal. They also have the same overall shape of the GP profile, with the only difference that the bath is larger than the piston. As shown above, coupling two systems with the same temperatures 

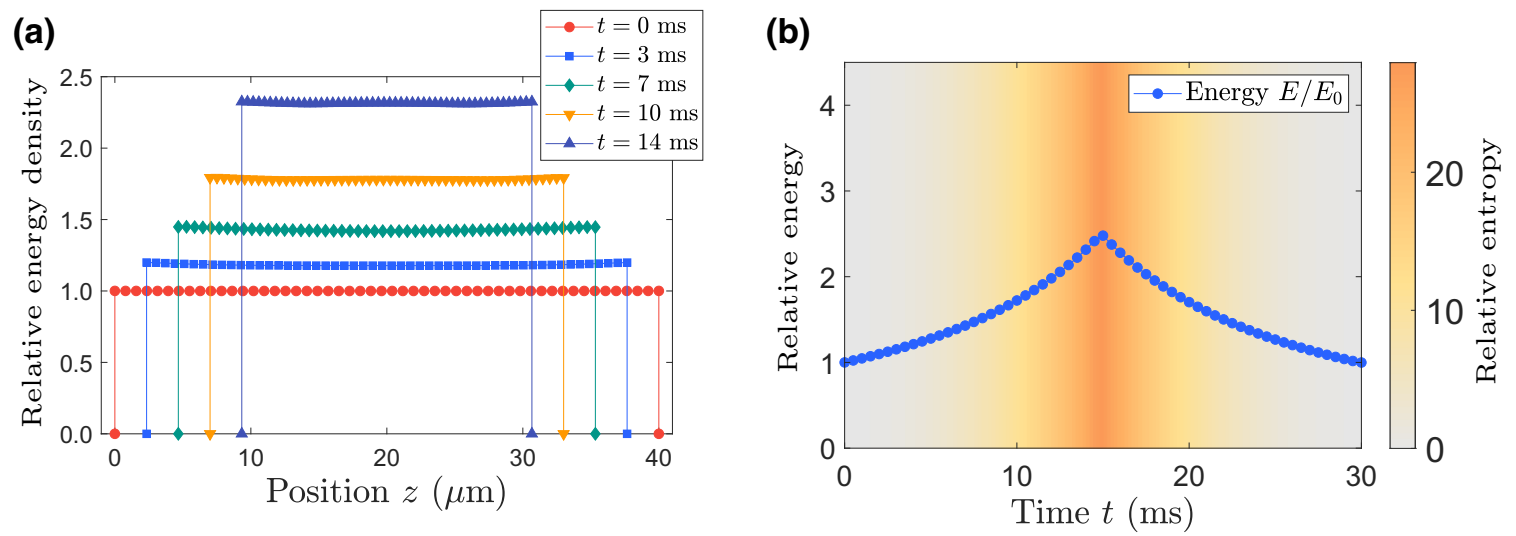

FIG. 4. Single stroke of a piston. A condensate of length $40 \mu \mathrm{m}$, initially thermal and homogeneous is compressed to half length within $15 \mathrm{~ms}$ and then re-expanded to its initial length in the same time. (a) Energy density during compression. The piston keeps a homogeneous energy density that increases when compressed due to increasing pressure of the gas. (b) (Non)equilibrium properties of piston. We plot over time the total energy relative to its initial value (blue dots) and the relative entropy to the closest thermal state as a color gradient during the compression and decompression. The piston goes out of equilibrium, as the relative entropy to the closest thermal state increases during compression. The reverse happens when the piston decompresses - it is again fully thermal at the initial energy and temperature at the end.

does not lead to heat flow. However, after the piston is compressed its energy is higher and so is its effective temperature. This creates an effective temperature difference between piston and bath, which, using then the valve QTP, enables heat flow from the piston to the bath. After this heat flow is completed we close the valve and decompress the piston to its initial length, and note from Fig. 5(a) that it becomes colder than it has been initially. Figure 5(a) shows the results of this protocol plotting the full spatiotemporal dynamics of energy density. In Fig. 5(b) we show that the compressed piston couples to the bath with effectively squeezed modes, so that the two systems are not at thermal equilibrium while the valve is open. Nevertheless energy in the piston decreases, due to heat flowing into the bath, which is seen in Fig. 5(a) in a form of a light-color stripe entering the bath. Finally, we find that the total energy in the piston decreases to a lower value than initially, thus we conclude that the piston has been overall cooled down. At the end of the protocol the decompression undoes the squeezing of the modes and the piston essentially comes back approximately to thermal equilibrium, signified by a low relative entropy to a thermal state.

Summarizing, we have performed work on the piston, which therefore allowed us to enable heat flow between
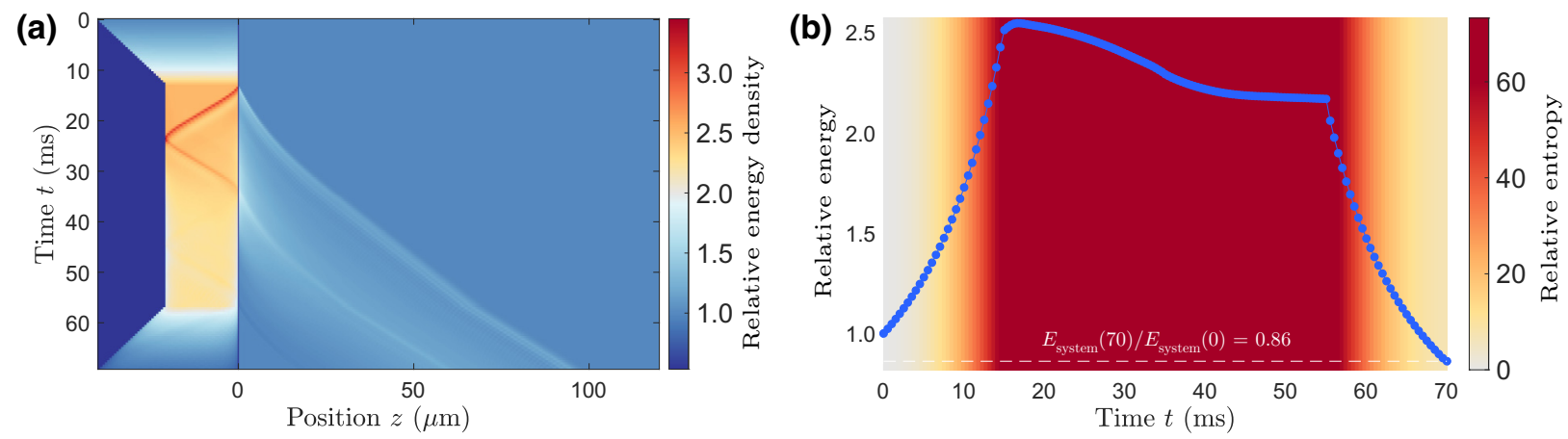

FIG. 5. Heat flow between the piston and bath. We consider the piston and bath being two initially independent condensates, with the bath being 3 times larger in size. The piston on the left is compressed to half of its original size. We then couple the piston to the bath at $t=15 \mathrm{~ms}$ and start decoupling them right after. (a) Energy density over time. We plot the energy as a color gradient in a space-time grid. The coupling between the two parts introduces the propagation of wave packets at the speed of sound, which is higher in the piston, due to the higher density resulting from compression. (b) Energy dynamics in nonequilibrium. We plot the ratio of average energy versus initial energy in the piston over time. We observe that it first increases strongly, while decreasing to a value that is less than 1, just before the piston starts coupling with our system of interest. This is what will allow us to cool the system with a full Otto cycle. We also plot the relative entropy to the best fit thermal state as a color gradient in the background and observe that during coupling the system goes strongly out of equilibrium, while returning to be close to equilibrium at the end. 
condensates. By composing the compression QTP with the open valve QTP, we demonstrate that is is possible to deposit some of the piston's energy into the bath.

\section{COMPOSING QUANTUM THERMODYNAMIC PRIMITIVES TO BUILD A QUANTUM FIELD REFRIGERATOR}

The challenge one faces studying cold atomic gases experimentally is that all methods of cooling eventually always reach a limit once the temperature is small enough. In the ultracold regime the last resort is to let some of the atoms escape the trap. Ideally one would like that (1) those particles leaving the system to be preselected such that they carry above-average energy, and (2) the gas left behind rethermalizes [68]. These two ingredients make up evaporative cooling. However, in one-dimensional systems they cease to apply, due to the change in scattering properties [69-72]. Nevertheless, in Ref. [73] the effect of letting atoms escape by applying an additional $\mathrm{rf}$ field has been explored in the 1D regime: at extremely cold temperatures it has been demonstrated that cooling continues, and its limits were also quantitatively mapped out.

The intensities and detuning of the applied rf field ensures the energy-independent loss of atoms [73]. This observation has suggested a successful modeling approach to the process by the phononic Hamiltonian (5) whose density parameter $\rho_{0}$ decreases over time according to the atom loss rate. Within this model the energy gets decreased due to this change in the Hamiltonian. By assuming that the uniform atom-loss process is sufficiently slow, the dynamics of phononic modes has been used to theoretically explain why the system is left approximately in thermal equilibrium with a decreasing temperature [73], see Refs. [74-76] for additional discussions.

By an analytical treatment, which assumed that (1) the atom-loss process is adiabatically slow, (2) only phonons rather than particlelike excitations are involved, and (3) shot noise has a negligible contribution, this model leads to the relation

$$
T^{\prime} / T \approx\left(\rho_{0}^{\prime} / \rho_{0}\right)^{3 / 2}
$$

In harmonic confinement, the peak density is $\max \left(\rho_{0}\right) \propto$ $N_{\text {atoms }}^{2 / 3}$, which together with Eq. (21) yields qualitative agreement with experimental observations: the coldest temperature reached is found to depend linearly on the number of atoms,

$$
T \propto N_{\text {atoms }}
$$

Thus, the temperature of a condensate can be lowered by allowing for more atom losses; however, this dilutes the system and cannot be continued indefinitely, otherwise quasicondensate properties will be lost [28]. Equation (21) is also valid for a boxlike confinement, where the temperature dependence on atom number is expected to be nonlinear.

References [34,73] give representative values for cooling in a harmonic trap. For state-of-the-art data reached with boxlike confinement [33], $N_{\text {atoms }} \approx 5000$ confined atoms can form an approximately homogeneous condensate of about $L=50 \mu \mathrm{m}$, and the estimated temperature is $T \approx 50 \mathrm{nK}[17,33]$. Summarizing, uniform atom losses do lead to cooling, but this does not follow the usual mechanism of rethermalization via scattering as typically seen in evaporative cooling. Rather, this is a direct consequence of decreasing the density parameter in the phononic Hamiltonian.

In general, the effective barrier of Eq. (22) seems hard to overcome. The current achievable lowest temperature for a fixed prescribed density on the system is limited by the initial density and temperature of the gas accessible from previous stages of cooling (laser operated); and this initial density cannot be infinitely large, given the constraint of operating in the quasicondensate regime. Moreover, the evaporative cooling will eventually either exhaust the available atoms diluting the system (effectively leaving the quasicondensate regime), or completely lose its efficiency in the sense that the evaporation has negligible cooling effect due to infinite thermalization time. One therefore requires novel cooling methods to overcome this impasse.

\section{A. Cooling by escaping atoms within QTP framework}

In this section, we point out that cooling by uniform atom losses, which is the state-of-the-art cooling technique for one-dimensional gases in the lowest temperature regimes, can be conceptually captured in the QTP framework. In particular, consider a sequential concatenation of a dilution of the system and possibly coupling to a cold bath. The continuous dilution that arises from atoms escaping the system can be conceptually modeled by the piston and valve QTPs. Indeed, if during cooling we have the gas of $N_{\text {atoms }}$ atoms uniformly occupying the interval of length $L$, then after one particle escapes the linear density will change according to

$$
\rho_{0}=\frac{N_{\text {atoms }}}{L} \rightarrow \rho_{0}^{\prime}=\frac{N_{\text {atoms }}-1}{L} .
$$

However, the same can be achieved by the system behaving like a piston of size $L$ expanding by $\Delta L$, such that the linear density changes according to

$$
\rho_{0}=\frac{N_{\text {atoms }}}{L} \mapsto \quad \rho_{0}^{\prime \prime}=\frac{N_{\text {atoms }}}{L+\Delta L} .
$$

To complete the description we can impose $\Delta L$ to be such that $\rho_{0}^{\prime \prime}$ equals $\rho_{0}^{\prime}$. Additionally, we imagine placing a valve to be positioned at $x=L$ at the edge of the piston, so 
that when it expands to $L+\Delta L$, atoms exit the valve into vacuum, and after we close it the remaining system has the same density $\rho_{0}^{\prime}$ and the length is $L$, exactly as in evaporative cooling. In other words, in our modeling, the piston lowers the density by operating at constant particle number $N_{\text {atoms }}$ and varying length $L$; while in evaporativelike cooling, the change of density occurs at constant length $L$ but varying particle number $N_{\text {atoms }}$. However, on the level of intensive thermodynamical quantities, both are the same and this observation is implemented by the fiducial valve shutting of the $\Delta L$ portion of the expanded piston.

As a remark, the QTP framework can be also used to capture evaporative cooling involving a rethermalization process. When evaporative cooling is most effective in its operation, only the atoms that individually carry above average energy leave the system. This way of cooling is more efficient as each escaping atom carries on average more energy than an atom remaining in the system does. This can be modeled in the QTP framework by opening and closing a valve coupling the system to a cold bath. The heat flux and timing jointly govern the exchange of energy between the system and bath; they should be chosen such that the lowering of the system's energy is the same as an evaporating atom would do.

\section{B. Cooling by atom-number dilution with a subsequent recompression to restore atom density}

Cooling facilitated by atoms escaping the trap irreversibly dilutes the system. As explained above lowering the temperature of the atoms at a given density being fixed is the right way to compare different cooling approaches. As anticipated in Fig. 1 running refrigeration cycles like in a machine can be expected to lead to cooling without changing the atom density. However, it is also true that in a QFM as presented in Fig. 1 there are two subsystems, acting as the piston and bath, which are constituted by a sizeable amount of atoms. The question then arises: can any advantage be gained when aiming to cool at prescribed density in simply evaporating these systems?

While such a question is quite general, let us discuss it by formulating a representative protocol whose analysis will suggest an overall answer. First of all, if the dilution has to have any effect on the system we must allow for contact with the subsystem that we would like to cool at constant density. One way to achieve that is to consider the entire system (system, piston, and bath in Fig. 1) to be uniform, then cool it down by dilution implemented by the escaping atoms, and then use the piston QTP to compress the system back again to restore the density to the initial value. The idea here is that the evaporation of the amount of atoms taken up by the piston and bath should lead to cooling and after the compression the system should have the prescribed density.
However, we can anticipate that this effect will not lead to overall cooling. This is because the dilution cools down the system by reducing the density via the atom number but the compression heats the system up as should be in a gas and has been discussed in Fig. 4. Intuitively, on the phononic level this is seen by noticing that increasing the density, implemented by reducing the volume of the system, changes the Hamiltonian, which associates a larger energetic penalty to phase fluctuations. In other words, we first in a time-dependent fashion change the linear density to the same value by reducing the numerator (atom number) in its definition and then increase the density back to the initial value by decreasing the denumerator (system length). As long as we are in the phononic regime it does not matter which process changes the density - the modeling will be the same and both processes, that is cooling by atom losses and recompression, admit the same modeling using the phononic Hamiltonian so one should expect that they are mutually complementary.

In Fig. 4 we show that a stroke by compression and recompression is effectively reversible in that the overall phononic energy returns to its initial level. In the model it does not matter whether the density is changed by changing the atom number or the length of the system. For this reason, we expect the reversibility of the phononic energy change to be also valid when combining the dilution process by uniform atom losses to cool down with the piston QTP to restore the density. This can be verified in a future experiment to lay the ground for implementing a quantum field refrigerator based on the QFM involving the much more sophisticated approach using cycles and composing many QTPs together.

On the theoretical grounds supplemented by the empirical knowledge drawn from past experiments the case for refrigeration via the QFM seems to be clear: reducing the entropy per particle in a subsystem of a cold-atom system should be achieved by moving this entropy to a bath as in a QFM. Having said that, considering other interesting variants of combining processes such as atom losses and QTPs described here for problems of interest, cooling being one particular example, is available experimentally and can be further explored in the future. As we show next, if one aims to achieve cooling in a systematic way it is advisable to run QTP cycles in a QFM as illustrated in Fig. 1.

\section{Quantum field refrigerator: QTP cycles for sequential cooling and reduction of entropy of a subsystem}

In this section, we demonstrate how to compose the discussed primitives to perform a useful protocol, namely cooling. By simulating the quantum field refrigeration machine depicted in Fig. 1 at this density and temperature, we find a cooling cycle where the system temperature 
decreases, highlighting the usefulness of such a new active cooling protocol. The cycle works as follows.

(1) The machine is initialized by setting a system, a piston, and a bath to their respective thermal equilibria.

(2) The first nontrivial thermodynamic transformation is the compression of the piston with a subsequent interaction with the bath. The work inserted to compress the piston enables heat flow as shown above in Fig. 5.
(3) After decoupling the piston from the bath, the piston is expanded back to its initial length. This aims to cool it down and when it subsequently interacts with the system it should take up some heat from it.

(4) Finally, the piston and system are decoupled again and the cycle can be repeated.

In Fig. 6, we depict the energy changes of these three pieces of the QFM over the duration of the Otto refrigeration protocol obtained from a numerical simulation [77].
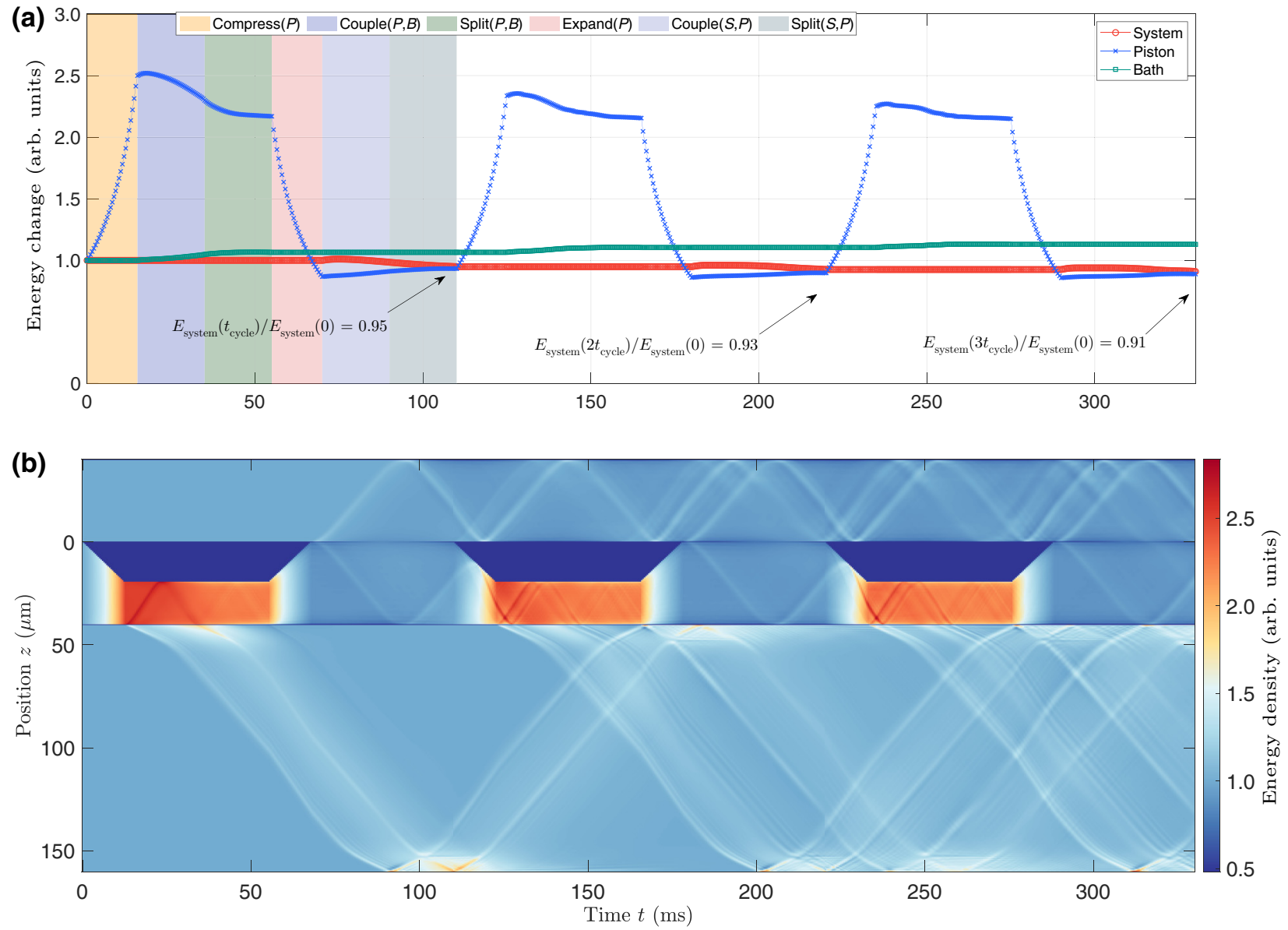

FIG. 6. Top: quantum field refrigerator. The QFM is initialized in thermal equilibrium and equal density, i.e., the system, piston, and bath differ only in length, which is $40,40,120 \mu \mathrm{m}$, respectively. We run the Otto cycle by compressing the piston (15 ms), depositing heat in the bath $(40 \mathrm{~ms})$, and then expanding the piston again $(15 \mathrm{~ms})$. The cooling begins at around $70 \mathrm{~ms}$ by coupling the initially thermal system to the cooled piston. The systems exchange energy by the physical mechanism of the valve described in Sec. IV A. After the final splitting of the system and piston, we find that the system cools down, while the quantum field refrigerator extracts approximately $5 \%$ of the system's initial energy. This drop in energy is large enough to be detected by existing experimental read-out methods. In this plot, one observes that further cycles continue to contribute to cooling of the system, but only in very small amounts. The currently used parameters are probably nonoptimal, and we anticipate improvements of the refrigeration efficiency via optimal control. This, however, will depend on the modeling of other details in the quantum simulation of this cycle. Bottom: time-and-spaceresolved energy dynamics during the operation of the QFM. From the top we show the system, piston (which changes in size) and bath. Whenever a valve QTP is operated, wave packets are injected and multiple reflections in each system can occur. The principal wave packet in the bath is timed to arrive at the interface to the piston at around $160 \mathrm{~ms}$ when the valve is closing, so that the piston energy is not further increased. The overall amount of energy in the bath increases, which is due to the presence of multiple wave packets. It is noteworthy that, depending on scheduling, the larger among two coupled systems can take up two wave packets. Hence, considering the piston to be substantially larger than the system could allow the removal of all excitations from the system. This is also why the bath takes up most of the wave packets. 
It can be seen that the piston first increases its energy due to compression $\left(t_{\text {comp }}=15 \mathrm{~ms}\right)$ and then lowers it during interaction with the bath and successive expansion $\left(t_{\text {merge }}+t_{\text {comp }}=35 \mathrm{~ms}\right)$. Finally, the piston increases again its energy when interacting with the system and then resizing to its original length (again $t_{\text {merge }}+t_{\text {comp }}=35 \mathrm{~ms}$ ). Overall, at the end of the first cycle $\left(t_{\text {cycle }}=110 \mathrm{~ms}\right)$, the piston has slightly decreased in energy, while system and bath have consistently decreased and increased their energy, respectively. By performing three Otto cycles, we obtain cooling of $9 \%$ in a total time of $330 \mathrm{~ms}$, which gives us an estimate of the cooling power of our QFM. However, we also observe that such cooling power actually decreases in subsequent cycles, thus raising the question of the ultimate limits of cooling for this machine. We discuss this interesting aspect further in Sec. VIB.

\section{Discussion of the engine: our estimates versus other prospects}

We consider rather conservative estimations for the parameters. Several ways to weaken the requirements can be explored in the experiment in order to obtain a higher cooling ratio. (i) As shown in the bottom panel of Fig. 6 the piston has been compressed to half its length, which ultimately limits the capacity of the machine to cool down. Performing more work and compressing the piston more would allow for further cooling. (ii) Modifying the barrier height and various other aspects of our QFM model, higher cooling ratios are possible as shown in Appendix C6. These among others could be to reset baths, coupling at higher density etc., which have features that depend on the particular implementation and hence cannot be completely anticipated theoretically ahead of performing the experiment. (iii) Let us remark that for the sake of simplicity and also for analogy with the usual thermodynamic Otto engine, the piston is the only component that changes size during the protocol. However, one can think of more general scenarios in which the bath is expanded while the piston is compressed, and afterwards, the system is compressed while the piston is expanded - after all in the experiment it is our goal to cool down the quasicondensate more than it is possible with existing methods and an unconventional quantum thermal machine with various elements changing their size would be helpful for this purpose. Summarizing, there are a lot of important points one can consider when devising a QFM. It is clear that once QTPs are realized, their conceptual clarity will be advantageous in order to appropriately compose them to achieve maximal possible cooling in the experiment.

\section{DISCUSSIONS AND FURTHER SCOPE}

While further developing the framework of QFMs and during the upcoming efforts to realize a QFM experimentally, numerous questions relating to the fundamental physics of the system and technological implementation beyond the scope of this initial Paper will have to be further investigated. Our discussions below highlight several aspects, which could invite expertise from fields such as engineering and quantum control of out-of-equilibrium quantum many-body systems to become particularly useful. Thinking ahead, the program of devising a QFM presented in this work is also expected to stimulate a range of further theoretical investigations in the field of quantum thermodynamics [1-7]. These will range from (experimentally inspired) studies of the role of information in quantum thermodynamics to prospects for further development of the theory of quantum thermodynamics from a quantum-information perspective.

\section{A. The role of information in the QFM}

If we could - fictitiously - precisely measure the manybody eigenstates of our complete machine, we could in principle achieve complete control about the system. Needless to say, in a quantum many-body system this is impractical and we have to restrict ourselves to physically relevant, local, few-body observables and a finite set of their correlations. Reference [20] provides an overview on how far one can presently experimentally go in such endeavors. These limitations will define what we can possibly know about the system and what we can hence make elaborate use of - and what we are bound not to be able to know and therefore need to ignore. In this section, we highlight several important aspects of accessing information and correlations and observing their roles in such a manybody QFM. The manipulation of one-dimensional quasicondensates via relatively simple yet highly controlled thermodynamic processes in the deep quantum regime seems to be an ideal test bed for such considerations.

\section{Correlations and anomalous heat flow}

An interesting future direction is the exploration of the question how strongly are the elements of the QFM correlated, how to quantify and control these correlations, and how to make use of them explicitly in the design of a QFM. The coupling and decoupling of two interacting many-body systems, i.e., the operation of the valve QTP, is a direct way to induce correlations or even entangle the two. The canonical example thereby is the double well, that has a physics similar to a beam splitter in quantum optics. When the decoupling is slower than the time scale given by the interaction energy, the two systems will build up quantum correlations, which persist even if they are separated [78-80]. An indication that this also works for the excitations in a many-body system described by an effective quantum field theory is the observation of number squeezing in the modes created by slow splitting [44].

The engineering ofa such correlations is an important question especially in the context of work extraction [81], 
since they may produce interesting dynamics. In particular, with the exhaustion of correlations, instead of inputting extra energy and work into the system, one can induce a reverse in what is called the "thermodynamic arrow of time," referring to a reverse in the direction of heat flow between two systems. Such a phenomenon is commonly referred to as anomalous heat flow [82,82-86]. Proof-ofprinciple experiments between qubits have been demonstrated, which involve the particular engineering of specific unitary processes to address a fixed, two-dimensional energy subspace [86]. There also exists experiments studying thermodynamic spin currents, which are blocked by the initial state preparation [87]. This blocking is anomalous but not in the sense that the current is reversed, for which correlations must be engineered appropriately. Thus an anomalous reversal of heat flow with a detailed experimental evaluation of the role of correlations in this process has yet to be worked out in detail for complex manybody systems in the quantum regime. This is an important question as it is not clear whether global, macroscopic operations are enough to generate (i) the right correlations, and (ii) dynamics that allow the emergence of such behavior.

Our simulations, on the other hand, predict that the process of merging two condensates creates the desired effect of creating correlations that will potentially lead to anomalous heat flows (see Fig. 7). We can quantify the amount of generated correlations by computing the mutual information between condensates $S$ and $P$, which is defined by

$$
I(S: P)=S\left(\hat{\rho}_{S}\right)+S\left(\hat{\rho}_{P}\right)-S\left(\hat{\rho}_{S P}\right)
$$

where we recall that $S(\cdot)$ is the von Neumann entropy of the quantum system. Note that nonmonotonous behavior of the mutual information can also be used as a signature of non-Markovianity [88,89]. For the Gaussian states in our study, these quantities are directly computable given the covariance matrices (see Appendix B). Furthermore, this can also be accessed in the experiments via tomographic data. We see from our simulations that the idle evolution of the joint many-body condensate is sufficient to produce periodic oscillations in the mutual information, in which a similar oscillatory behavior in the direction of heat flow (similar to an ac current) can be observed. It remains to verify how much of the change in mutual information is directly responsible for the reversal of heat flow. Not only this is a fundamentally interesting aspect to study by itself, but its natural presence in the working of the thermal machine also raises the question if one can use this heat flow to our advantage. For example, it is known that with correlations there is also the possibility of providing a way of implementing the extraction of macroscopic work probabilistically from a heat bath [90].
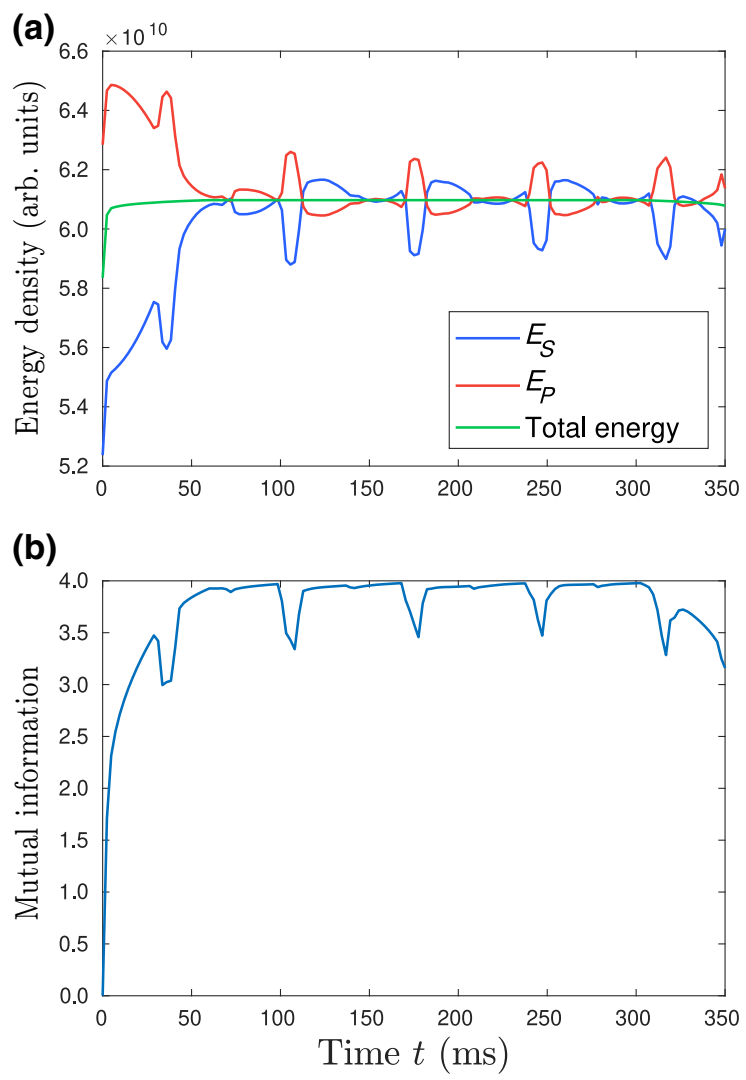

FIG. 7. Two systems, 30 and $40 \mu \mathrm{m}$ long, start uncoupled with temperatures of 50 and $60 \mathrm{nK}$, respectively. They are merged in the first $60 \mathrm{~ms}$ of the evolution; after that the coupled systems evolve for $240 \mathrm{~ms}$ and then are decoupled during $60 \mathrm{~ms}$. (a) Energy flow between the two condensates. After merging there is heat flowing in both directions. (b) Mutual information of the two condensates. The mutual information increases once the two condensates start interacting; after that it decreases for a short time and increases again to its maximum value, this happens when the energy is maximum in the condensate with a hotter initial temperature (and minimum for the other condensate), which shows that the mutual information is correlated with the reversal of heat flow.

In this light, it would be interesting, also in relation to earlier experimental works on cooling cold atomic gases with sequential operations [91], to reveal such quantum aspects of protocols of this type in near-future atom-chip experiments. References [37,44] have uncovered signatures of quantum noise and squeezing during longitudinal splitting of the quasicondensates. This is an exciting indication that it can be possible to reveal (with statistical significance) the presence of entanglement under similar conditions, e.g., quantum correlations between eigenmodes reflecting the effect of various perturbations that can be applied. The detailed study and controlled usage of these phenomena is therefore one of the future directions of immediate interest, which our platform of interest has a natural advantage of studying. 
For the simulations of the full quantum fridge (Fig. 6) we currently assume that dephasing occurs after we split systems, i.e., the correlations between the elements of the QFM are modeled to be lost every time splitting is completed. This should be understood as establishing a reference, first-case study where temperature fluctuations and dephasing due to long cycle times render the effect of correlations on the QFM operation to be small. This should then be compared with experiments, in order to understand the extent of how correlations influence the machine performance. Moreover, further lowering the currently accessible temperatures will allow the few phonon regime to be entered in which quantum vacuum fluctuations will certainly become manifest. These features are closely connected to entanglement in real space $[92,93]$ because the phononic vacuum is entangled in real space as it can be understood via arguments from conformal field theory [94]. In this regime, the thermal coherence length $\lambda_{T}$ will be comparable to the system size and phase correlations will decay polynomially instead of exponentially.

\section{Non-Markovian effects}

Besides anomalous heat flow, there are more generic non-Markovian effects, which our system can be used as a observational test bed $[88,89,95,96]$ on thermodynamic operations. Such dynamics originate from the intermediate size of the bath so that a backflow of information occurs. The presumably principal source of non-Markovianity is hinted at in Fig. 6, where we see that the wave packets injected by operating the valve get reflected from the boundaries of the system and come back to the position of their origin in finite time, in fierce violation of any meaningful Markov approximation. Notably, this effect should be expected to hold also in the presence of weak nonGaussian perturbations as various atom-chip experiments have already experimentally demonstrated that these features remain intact in close to integrable situations, also in the presence of nontrivial trap geometries.

In most works on quantum thermodynamics [97,98], an infinite bath is considered, but it is unclear under which conditions these modeling assumptions would be valid for the intermediate-sized baths in a QFM experiment. The studies of local recurrences can be seen as entry points to interesting theoretical studies of the possible repercussions of wave packets returning back to their origin in finite time.

Loss of information ultimately proceeds through dephasing of collective excitations. For quasicondensates these are phonons $[37,99,100]$, the dephased state emerges in a light-cone fashion [43], and is described by a generalized Gibbs ensemble [44], i.e., different modes can have effectively different temperatures determined by the state preparation. The long time behavior depends on the spectrum of these collective modes. If the atoms are confined to a box-shaped trap, then the phonon frequencies become commensurate, i.e., $\omega_{k}=\pi c k / L$, with $k=1,2, \ldots$ being the mode index, and recurrences are observable at short times [33,101]. This effect is a distinct source of nonMarkovianity from the localized wave packets returning to their origin in finite time and can occur even in a homogeneous system. As detailed in Ref. [17] the recurrence is a recurrence of the squeezed (momentum) modes, where each mode $k$ is represented by an ellipse in phase space rotating around the origin with frequency $\omega_{k}$ and all ellipses realign their axes as soon as the slowest $k=1$ mode rotates by a full angle. In that moment the $k=2$ mode will have made additionally one more full turn, and similarly higher modes too. In other words, due to the linear spectrum all modes realign. This pertains to eigenmode populations and the state in real-space can be homogeneous during the dynamics. This, however, does not occur in a harmonic longitudinal confinement with trap frequency $\omega_{\|}$, where the eigenfrequencies are nonlinear $\omega_{k}=\omega_{\|} \sqrt{k(k+1) / 2}$ [28] and are incommensurate. Still, when the entire system is engineered to be captured by few collective commensurate modes, non-Markovian behavior and significant memory effects can dominate the system dynamics.

Let us illustrate that with an example: the role of the reservoir in a thermal machine cycle will strongly depend on the design of the mode spectrum and on when the "contacts" take place. In other words, the timing of the valve QTPs will matter. If the recoupling is in between recurrences, the reservoir will appear dephased and with seemingly no memory of what happened during the previous cycle. However, the system is coherent: by changing the timing the valve coupling can occur at the time of the recurrence and the reservoir can appear to have memory of what happened during a previous cycle, and hence be a non-Markovian bath. Designing the longitudinal confinement in each part of the thermal machine will allow us to have in principle (nearly) full control of the memory of selected states in the thermal machine at later times. This will allow us to design and probe a large variety of interesting Markovian and non-Markovian situations [102-106].

\section{Finite-size effects due to energy fluctuations}

Individual realizations of the experiment are subjected to non-negligible thermal fluctuations. A particularly interesting question lies in observing the predictions related to finite-size effects derived in various theoretical frameworks of quantum thermodynamics. Our systems are small, and therefore can be heavily influenced by fluctuations in energy. Moreover, we are interested in a single-shot process of cooling, namely to run the machine for at most a few cycles for a single initial preparation; as opposed to preparing a large amount of identical condensates and seeking to cool them only on average. The performance of 
machines in such a single-shot setting has typically been captured by additional "thermodynamic laws," which are distinct from the standard laws that are valid in the thermodynamic limit. Such "laws" are essentially constraints, which have been phrased in terms of (i) generalized free energies in the context of a resource-theoretic language of quantum thermodynamics [107], (ii) fine-grained Jarzynski equalities [108], or (iii) other measures specifically tailored for Gaussian systems [109]. These are intricate and important theoretical descriptions. But make-or-break questions for the significance of such pictures presumably are the following ones: can we observe their predictions? Specifically, how relevant are they to characterize the potentials and limits of practical thermodynamic protocols such as the cooling scheme proposed in this work? Much remains to be explored in this direction for quantum manybody systems in contrast to other physical settings where specific ideas have been proposed [110].

\section{B. Efficiency of quantum machines: notions of work and performance versus theoretical limits}

Turning our attention to the notion of efficiency of quantum machines, we would like to connect the expected performance of our proposal to limits set in the literature. A couple of comments are in order before we begin this discussion. First of all, there are different notions of efficiency that one could discuss: on the one hand, the quantum efficiency would compare how much work is drawn from the quantum system in order to implement the machine operation. For a fridge that would be the coefficient of performance, simply given by the quotient of the heat removed from the target system and the work performed by the piston. On the other hand, the complete efficiency would be the quotient of heat removed by total work invested in keeping the machine running, i.e., including the power drawn by the computers, DMD, and other physical machinery that is needed to keep the system running as a whole. As the cost of control is generically orders of magnitude above the energy scale of the system, any complete evaluation of efficiency of a controlled quantum engine (such as we propose) would not be very meaningful, since running this machine as an engine to generate work would be futile: much more work would have to be put into the control as one could possibly expect to gain. The quantum efficiency on the other hand, does not have a great operational meaning unless supplemented by further context: from a pragmatic perspective, it is unclear why one should care only about the work that is specifically done by the piston and ignore all the work that went into generating the field defining the piston in the first place.

The main goal of the machine is to cool down a target system in ways that transcend the possibilities of purely classical refrigeration processes. Here, an exciting direction is to instead explore experimentally the fundamental limits of cooling, as they are usually captured in readings of the third law(s) of (quantum) thermodynamics. Adapting the terminology from recent works [111,112], the QTP toolbox can be seen as coherent building blocks for a thermal machine, whereas resource-theoretic operations are energy incoherent. Nevertheless, coherent operations (i.e., time-dependent Hamiltonian control operations) and incoherent operations (i.e., resource-theoretic operations), if both given the same amount of complexity, can achieve similar performance (final energy) in terms of cooling of qubit systems [111]. Therefore, the fundamental bounds obtained from resource-theoretic frameworks in machine performance $[107,113,114]$, especially such as the third law derivations [114,115], may be a valuable benchmark.

In all thermodynamical processes considered for our QFM in the future, an important issue is the notion of work itself. Indeed, quantifying work extraction in the quantum regime can be treated by various theoretical frameworks of quantum thermodynamics and might yield different results depending on the definition used [116]. For the refrigeration cycle that we propose here, however, the useful output of the QFM is easy to assess, as shown in Fig. 6. This, however, is not as straightforward in general for other tasks that may be implemented with the QTPs that we have presented. In that case, additional ideas for quantifying quantum work will have to be developed in accordance with our modeling involving exclusively unitary processes induced by time-dependent Hamiltonians. For example, since we always initialize the quasicondensates in a thermal state, this process is similar to the standard setting of fluctuation relations [117]. However, the statements of work extraction provided by fluctuation relations involve initial and final projective energy measurements on the system, which is not directly measurable in experiments with quantum many-body systems.

QTPs can be used to perform work on systems and in the process we saw that this brings them out of equilibrium. Therefore, in the resource-theoretic framework of quantum thermodynamics $[107,118]$ they should be interpreted as being resourceful and hence stand in contrast to free operations and states, which are usually studied in this formalism. This highlights the gap between this powerful, but abstract, framework with what is meaningfully achievable in experimental setups. So far, the energetic worth of nonthermal resource states has been studied in the context of distillation rates $[119,120]$. Our platform will provide a test bed for quantifications on the level of operations rather than states and might be potentially useful for practical settings.

\section{Experimental realization of a QFM}

The first type of energy referring to known properties can in the widest sense be related to work, the latter to heat and entropy. Ultimately, it is the amount of information one has about the energy present in the system that decides 
whether it should be interpreted as heat or work $[1,113$, 121-125]. Aside from the conceptual issue of separating work and heat, much progress has also been made in theoretical quantum thermodynamics, in terms of predictions of how energy exchange in finite-sized quantum systems would occur.

In the present Paper, we deliberately focus on a simple and straightforward way of implementing a QFM in the form of a one-dimensional bosonic quantum gas, which can be described with a Gaussian effective model. Having said that, there are many interesting directions that can be explored in order to extend our proposal.

\section{Non-Gaussian QFMs}

We have left it open to what extent higher-order, nonGaussian contributions will play a substantial role in the operation of the QFM in the experiment. One example where these could potentially matter is when running the QFM with a long cycle time. This is because of dephasing or damping effects, which are not present in the TLL model can occur in real experiments already around $50 \mathrm{~ms}$ $[17,33]$. We currently take into account effects of dephasing only whenever two systems are split, which is consistent with this time scale since the merge-split protocol considered in our simulation is $40 \mathrm{~ms}$. A key aspect of future investigations will be to comprehensively explore weak non-Gaussian effects arising from such effects. We expect many-body dephasing to primarily have the effect of thermalizing the bath, but otherwise not obstructing the heat flow, which occurs faster than the onset of any dephasing observed so far. Nevertheless, a detailed study will provide more substantial insights into this important aspect.

The second interesting case is to notice that during the splitting and recombination, the mean density at the interface is low and there the linearized phononic description might break down. This may lead higher-order interactions to become substantial and induce scattering of phonons around the interface. In the experimental implementation, one can extract higher-order correlations and study nonGaussian correlations [20]. If present, they can be studied by numerical field theoretic calculations [126] or compared with predictions based on fundamental relations in quantum thermodynamics [127].

Finally, the existing atom-chip platform allows one to controllably add sine-Gordon interactions [20,23] and hence also non-Gaussian QFMs can be explored experimentally. The sine-Gordon model is paradigmatic for our understanding of quantum field theory [128-131] thanks to its rich physics, e.g., excitations of finite mass and nontrivial topological properties. The experimental implementation [20] following the quantum simulation proposal from Ref. [23] has been realized using two longitudinally tunnel-coupled one-dimensional quasicondensates. In this case, the system should be described by relative degrees of freedom, the relative phase $\hat{\varphi}_{\text {rel }}(z)$ and density $\delta \hat{\varrho}_{\text {rel }}(z)$ fluctuation fields. These fields are obtained by considering the difference of the respective fields of each of the condensates, see, e.g., Ref. [33] for a detailed discussion in relation to a recent experiment. Using interferometric measurements $[20,33,34,65,66]$ correlation functions of the relative phase can be measured, which allowed substantiation of the fact that the physical system has been correctly described by the effective sine-Gordon Hamiltonian for two adjacent quasicondensates

$$
\begin{aligned}
\hat{H}_{\mathrm{SG}}= & \int d z\left\{\frac{\hbar^{2} \rho_{0}(z)}{4 m}\left[\partial_{z} \hat{\varphi}_{\text {rel }}(z)\right]^{2}+g \delta \hat{\varrho}_{\mathrm{rel}}(z)^{2}\right\} \\
& -\int d z 2 \hbar J \rho_{0} \cos \left[\hat{\varphi}_{\mathrm{rel}}(z)\right],
\end{aligned}
$$

with effective field operators now capturing the relative phonon modes and interacting with a non-Gaussian cosine term. Tuning of the tunnel coupling $J$ is possible experimentally, which would allow building QFMs in various interaction regimes, ranging from a system of noninteracting modes to a strongly correlated quantum system with topological excitations. See Ref. [16] for further details in the context of thermal machines and Ref. [20] for a detailed experimental study of the many-body aspects of the model.

Summarizing, the two coupled one-dimensional quasicondensates will allow us to build and study strongly correlated QFMs, where the degree of correlations (that is, the degree of higher-order correlation functions that are relevant) can be experimentally tuned. It is known that the time evolution of interacting local quantum systems is computationally hard (technically speaking, it is bounded-error quantum polynomial time (BQP) complete in worst-case complexity), and in practice computationally demanding for classical computers for physically relevant problems. This applies as well to the equilibrium processes involved in the operation of the QFM. While numerical studies may prove inefficient, the properties of these strongly correlated QFMs can be probed experimentally in detail by measurements of (higher-order) correlation functions $[20,132]$.

\section{Matter-wave interferometry of parallel QFMs}

An intriguing idea is to run machines in parallel. This opens up the possibility to compare the operation of two identical machines by direct observation of matter-wave interferometry [133]. On the atom chip it is possible to conceive of two machines positioned side by side, parallel to each other (Fig. 1 would then be the side view of two machines) and they would be identical in the sense of having the same initial state preparations and subsequent control operations implementing QTPs making up the Otto cycle. This can be done using well-established 
protocols of manipulating the gas using a longitudinal double well and interference has been observed in this case in various situations $[15,20,33,37,65,132]$. Interferometry by its nature looks at relative fluctuations and hence disregards classical disturbances in the operation, which are identical for both systems and directly measures quantum fluctuations. Their appearance should be studied interferometrically for various initial states of the two systems including the following.

(i) Two independent systems created by cooling two cold atomic clouds separately. This provides the base case to be compared to when studying more interesting initial states.

(ii) Two systems that are dephased in a prethermalized state [37]. In this case the temperature of relative degrees of freedom has been found not to be fully determined by the cooling process but rather to be related to the tunnel coupling $J$ in Eq. (26) present during state preparation.

(iii) Two systems with (nearly) identical phonon modes with strongly suppressed quantum noise in the relative degrees of freedom. Such states have been achieved experimentally $[44,80]$ and can be further improved by optimal control of the splitting process [134].

Each of these approaches would prepare machines that would have distinct initial conditions and an experimental study would allow insights to be gained on how these influence the operation of the QFMs. Observing features where cases (ii)-(iii) would differ from the simple case of independent machines $(i)$ would then most likely require a nonclassical explanation.

\section{Necessity of optimal control for operations of the QFM}

In our present study we have involved only very simple protocols to operate the different primitives building a QFM. In a real-world implementation, one would naturally like to speed up and optimize the different steps of a QFM. This should be in fact expected to be a crucial matter. This can be done by implementing optimal control methods [135-138], such as those reported for splitting a double well in Ref. [134] or for the excitation process in Ref. [139]. Notably, some of the control theory is already established for the piston QTP and has been successfully implemented [53]. There, a harmonic trapping potential has been considered and the extension of the GP profile has stably been modified. This involved the fact that modifying a harmonic trapping potential acts essentially as a lens for the individual atoms making up the system, so not only one can compress them appropriately but also accurately decelerate them when needed.

\section{Diagnostic tools for QFMs}

Finally, a particularly important direction to study is the development of further diagnostic techniques for the system along the lines of recent developments [17,20,34, 47,58-66,140-142]. We have discussed in Sec. III C and Appendix A the current experimental read-out capabilities and have proposed how to enhance them by novel variants of tomographic data analysis [17]. Detailed monitoring of the $Q F M$ will be crucial and novel hardware solutions can aid that goal.

A particularly interesting possibility is to trap a threedimensional condensate close to the one-dimensional QFM and use it as a sensing device. Matter-wave interference between two systems of different dimensionality seems to be interesting in its own right offering to study a wealth of various physical phenomena $[66,141]$. In addition, it could be expected to provide additional read-out resources with the goal of circumventing the current imaging resolution limitations that are difficult to improve otherwise. The implementation of this scheme would have the advantage of providing a direct measurement of the phase along of a single quasicondensate in contrast of the indirect tomographic approach. See Ref. [47] for related work in this direction and additionally Refs. [7,143] for a discussion of possibilities for immersion cooling.

Finally, let us remark about the possibility of performing nondestructive measurements, which are essential, e.g., for an analysis of a thermodynamical process using fluctuation relation theorems, which involves a two-step measurement process on the same system. Currently the measurements performed in experiments using the atom chip are destructive, see, e.g., Ref. [66] for a discussion of measurements following a time-of-flight expansion. When experimenting with 1D systems destructive measurements are experimentally easier because of the small atom number. In that case one can illuminate the complete system for readout and every atom scatters many photons. Measuring in time of flight has the additional advantage that the atomic cloud, which is initially only a few $100 \mathrm{~nm}$ in transverse size, can expand transversely to a size that is above the resolution limit of the imaging optics (as used in Refs. [144,145]). For a long time of flight, the atoms have moved away from the atom-chip elements that were being used for the control in the experiment, thus reducing spurious light scattering that can contaminate the pictures. Such a measurement is destructive in two ways: (1) the gas is released from the trap, (2) as the atoms are heated up so much by the light scattering that the low-energy quasicondensate description for the atomic cloud is not valid anymore: the BEC evaporates unless the system is large [146-151]. Thus, nondestructive measurements in our system can not be performed by illuminating the atoms for readout multiple times but rather in a less disruptive way, e.g., by out coupling of atoms [147]. Here, one would like to remove selectively atoms from a portion of the system and measure these projectively away from the system. This has the advantage that the system will not be destroyed and the measurement can 
be repeated. Additionally, imaging individual out-coupled atoms allows consideration of quantum-limited measurements $[152,153]$. However, the mechanical effects of even the second-order Zeeman effect in the strong magnetic field gradients of the chip traps, which are still on to keep the remaining system running, make these measurements more difficult. Still, these engineering challenges could be overcome in near term.

For applications in quantum thermodynamics and fluctuation relation theorems, it should be noted that these measurements would be local in space (product measurements of commuting observables). This is in contrast to many protocols assuming projective measurements in the entangled and nonlocal energy eigenbasis - in general it is not clear how to achieve these experimentally demanding requirements in quantum many-body systems.

As a final outlook, let us remark that measurements using outcoupled atoms could potentially allow for implementing error mitigation for the refrigeration QFM: when merging two systems, the number of excitations will be influenced by phase diffusion of the phase zero mode and the measurement of the relative phase between the outcoupled atoms could allow selection of the experimental runs that happen to have fewer excitations than the average realization. Assessing the back action on the system and the influence on the performance of the QFM in such a scheme is an interesting question for future study.

\section{Relation of our proposal to other platforms}

At the heart of this work stands the design of a specific QFM based on continuous cold atomic programmable potentials, for good reason, as this is a blueprint for a quantum thermal machine following the desiderata that we have laid out. In this sense, we see this specific choice rather as a strength of this proposal and not a weakness.

Having said that, it should be clear that ideas of creating a toolbox of thermodynamic primitives as macroscopic operations over quasicondensates, reminiscent of operations acting on bulk systems in conventional thermodynamics, may well carry over to other cold atomic platforms, stressing the generality of the approach taken. Specifically, for systems of cold atoms in optical lattices, digital mirror devices have been used to implement programmable potentials $[154,155]$. In such settings, giving rise to programmable Bose-Hubbard dynamics, a coupling and decoupling giving rise to a valve and the compressing and decompressing realizing a piston could be realized, following the general prescription of this work.

On a related but different note, we mention the relationship of the present proposal to other proposals of quantum thermal machines or refrigerators that have been put forward. Indeed, cold atomic quantum thermal machines have been considered [7], investigating the refrigeration of an atomic cloud, but not following the mindset of operational primitives laid out here. There, the use of two atomic species has been suggested, in which one atomic species implements the working medium and the other implements two baths that are hot and cold, respectively. Our proposed system is simpler and therefore presumably more robust against experimental uncertainties and imperfections than that of Ref. [7], which requires a very high level of control over the system and very precise fine tuning of experimental parameters. The recent work Ref. [156] starts with a gas of rubidium atoms cooled down to low temperatures, where the thermal machine, however, consists of individual cesium atoms and does not operate in the quantum many-body regime. Work already mentioned above demonstrates thermoelectricity in a fermionic ultracold atoms channel, connected to two reservoirs [91].

Further away still from the setting we consider here are proposed refrigeration schemes based on a phononpumping mechanism in nanomechanical systems [157, 158]. These schemes also aim at achieving cooling of a quantum system, albeit in a quite different way from the setting considered here. In that work, noninteracting phonons are suggested to provide the work fluid, in contrast to massive atoms that are in the focus of attention in the present work. The phonon number is not preserved and a nanomechanical system is expected to be open. This observation - together with the fact that the piston is anticipated to be realized as a traveling lattice perturbation acting as a semireflective barrier-seems to come along with substantial experimental challenges. There are also similarities, in that cycles involving three subsystems are being considered. In the present work, the cycles are composed of operational primitives involving massive and potentially interacting atomic quantum systems.

The most significant contribution of the present work is to carefully introduce and discuss these basic operations at hand of numerical results based on an accurate microscopic model, for which the cyclic processes in quantum thermodynamics devised are more an example than an aim in its own right.

\section{CONCLUSIONS}

In this work, we have set out to devise a blueprint for a genuine quantum thermal machine in one-dimensional ultracold atomic gases, a platform that we propose to realize complex thermodynamic tasks. We have proposed a quantum field machine (QFM) involving phononic degrees of freedom described by an effective quantum field theory. When devising this blueprint, resorting to a quantum mechanical description has been crucial to reduce the physical description of the system to a point where the functioning of the machine can be easily grasped. In order to provide guidance towards constructing thermal field machines, we characterize a toolbox of thermodynamic 
primitives, which are macroscopic operations over quasicondensates, reminiscent of operations acting on bulk systems in conventional thermodynamics. Our proposal puts forward a scheme for a refrigeration QFM that involves a system featuring quantum effects, a cold atomic gas, and the machine performs a useful task-cooling of phononic quantum fields. In contrast to previous realizations of quantum engines this cannot be practically achieved by controlling every single degree of freedom of the system as there are just too many. It goes without saying that this task is useful and we hence fully accommodated the three requirements that we have set in the outset of this work for a thermal machine to be a genuine quantum machine. We found that quantum effects present in our QFM are currently detrimental to its cause: operating the valve of the QFM induces inevitable excitations adding thermal noise of reservoirs. This is rooted in the quantum effect of phase diffusion of phase zero modes and in the dynamical Casimir effect and features a detailed temporal structure thus far ignored. Remarkably, even after accounting for realistic "imperfections" expected in the experiment we predict notable cooling. As detailed, exploring further quantum features is possible, including (i) at sufficiently low temperatures entanglement or zeropoint fluctuations leading to sub-Poissonian noise when operating the valve [37], (ii) non-Gaussian QFMs [20], (iii) non-Markovian QFMs [33], (iv) parallel machines amenable to measurements using matter-wave interferometry $[20,33,37,44,159]$, (v) quantum phase diffusion and phase-locking via Josephson oscillations [159], (vi) few phonon regime similar to quantum optics in the few photon regime where the quantized nature of the energy spectrum becomes manifest and individual runs of the QFM will unavoidably fluctuate.

It is clear that this work constitutes only a commencing study of a research program of a larger scope. We perform classically efficient numerical simulations, but calculations for a non-Gaussian QFM are expected to hit the computational complexity barrier: it is key to our work that the blueprint for a QFM devised here resorts to a quantum many-body regime, in contrast to work that aims at understanding single-atom heat machines [160]. Even though our operational principles and cycles are reminiscent of those of classical heat engines, i.e., canonical thermodynamical transformations, we highlighted some interesting issues obstructing understanding the functioning of our QFM using resource theories. We have encountered quantum features which in the future should, to the contrary of our current observations, be seen not as a burden but as a potential advantage: they should be used to improve the performance of the thermal machine in the deep quantum regime.

Our theoretical model for a Gaussian QFM is expected to largely capture the qualitative operation of the QFM. The quantitative features may change and there is a rich number of entry points for non-Gaussian behavior to set in. We expect their effect to be small and to not overhaul our predictions. Ultimately, whether this will play out to be true in reality can only be decided by performing an experiment.

We firmly believe that such a machine can and should be built, which will deepen our understanding of thermodynamics in the quantum regime. Further progress in the research field of quantum thermodynamics necessitates the development of useful quantum machines to drive, motivate, and guide the theoretical development of the corresponding laws, just as the advent of steam engines propelled the development of thermodynamics in the 19th century. It is our hope that the roadmap laid out in this work will serve this cause well.

\section{ACKNOWLEDGMENTS}

We are grateful to Fred Jendrzejewski and Spyros Sotiriadis for useful discussions. J.E. and J. Schmiedmayer are supported by the DFG Research Unit FOR 2724 on "Thermal machines in the thermal world". J. Schmiedmayer, J.E., and M.H. have also received funds from the FQXi (FQXi-IAF19-03-S2) within the Project "Fueling quantum field machines with information", for which the present effort is key. M.H. and G.V. acknowledge funding from the Austrian Science Fund (FWF) through the START Project No. Y879-N27 and the Lise-Meitner Project No. M 2462-N 27, M.H. and J. Schmiedmayer acknowledge the ESQ Discovery Grant "Emergence of physical laws: from mathematical foundations to applications in many body physics". J. Sabino acknowledges funding from the Austrian Science Fund (FWF) through the DK CoQuS. J.E. acknowledges funding from the DFG CRC 183 (Project No. A03), the European Union's Horizon 2020 research and innovation programme under grant agreement No. 817482 (PASQuanS). N.N. acknowledges funding from the Alexander von Humboldt foundation, and the Nanyang Technological University, Singapore under its Nanyang Assistant Professorship Start Up Grant. I.M. acknowledges the support by the Wiener Wissenschafts- und Technologiefonds (WWTF) via Grant No. MA16-066 (SEQUEX). J. Sabino, M.P., and Y.O. thank the support from Fundação para a Ciência e a Tecnologia (Portugal), namely through Project No. UIDB/EEA/50008/2020. J. Sabino acknowledges the support from the DP-PMI and FCT (Portugal).

\section{APPENDIX}

This appendix provides sections accompanying the discussion presented in the main text as follows. We begin by giving in Appendix A more details about the precise quantities that can be measured in experiments on the atom chip and discuss how to connect these to thermodynamical quantities. Next, in Appendix B we summarize 
the essential ingredients of the bosonic Gaussian formalism, which is the analytical base for the numerical code that produced our results. Finally, Appendix $\mathrm{C}$ provides an extended discussion on simulation details, including precise formulation of the lattice approximation employed in the code, extended description of the valve QTP [including how to compute the energy density or compare to the continuum limit in the scenario of sudden (quench) merging], of the piston QTP (including additional discussion of the model, compression dynamics and details of coupling inhomogenous QFTs after compression) and finally we discuss different relaxations of parameter constraints that have yielded an almost $30 \%$ cooling ratio.

\section{APPENDIX A: EXPERIMENTALLY MONITORING THERMODYNAMIC TRANSFORMATIONS IN PHONONIC QUANTUM SIMULATORS}

Let us begin by discussing which quantities, if measured experimentally, would reveal insights about the thermodynamic transformations in the system. We then proceed by explaining what are the direct experimental observables and how to connect to the desirable thermodynamical observables. In experiments, one should distinguish the cases of having a single quasicondensates and two which are adjacent. First let us discuss the former case where we have access to measurements of the atom numbers locally by transversal density absorption imaging (from the side). These numbers will be ultimately binned together due to finite resolution. The recovered atom number per bin $N_{i}\left(z_{j}, t\right)$ will fluctuate randomly between realizations $i$ and will give spatially resolved data where $z_{j}$ can be measured in steps of about $\Delta z_{\text {res }}=2 \mu \mathrm{m}$ on the atom chip. The quantities obtained for this lattice can be compared to theory by convoluting the continuum quantities by a Gaussian function with $\sigma_{\text {res }}=3 \mu \mathrm{m}$ and evaluating [17,34]. After taking this data at a given time $t$ one can obtain the density fluctuations as follows. The empirical mean of the observable random variable $N_{i}\left(z_{j}, t\right)$ gives access to the GP profile

$$
\rho_{0}\left(z_{j}, t\right) \approx \frac{1}{M} \sum_{i=1}^{M} N_{i}\left(z_{j}, t\right) .
$$

After subtracting these values from the individual realizations and squaring the shifted random variable we obtain the estimator

$$
\begin{aligned}
\Gamma^{\rho \rho}\left(z_{j}, z_{j^{\prime}}, t_{i}\right) \approx & \frac{1}{M} \sum_{i=1}^{M}\left[N_{i}\left(z_{j}, t_{i}\right)-\rho_{0}(z)\right] \\
& \times\left[N_{i}\left(z_{j}^{\prime}, t_{i}\right)-\rho_{0}(z)\right] .
\end{aligned}
$$

Indeed, what we obtain by this is nothing else than the estimate the second moments of density fluctuations away from the mean density of the quasicondensates. The on-site correlation gives information about the energy of phonons. As discussed in Sec. III in the main text, the total energy in the system can be obtained by considering the formal expression for expectation value of the Hamiltonian

$$
\left\langle\hat{H}\left[\rho_{0}\right]\right\rangle=\int d z\left\{\frac{\hbar^{2} \rho_{0}(z)}{2 m}\left\langle\left[\partial_{z} \hat{\varphi}(z)\right]^{2}\right\rangle+\frac{g}{2}\left\langle\delta \hat{\varrho}(z)^{2}\right\rangle\right\} .
$$

Of course in the experiment one can measure only at discrete positions but what we can do is try to obtain this quantity via a finite Riemann sum, specifically in the density sector we find

$$
E_{\rho}(t)=\frac{g}{2} \int d z\left\langle\delta \hat{\varrho}(z)^{2}\right\rangle \approx \frac{g}{2} \sum_{j} C^{\rho \rho}\left(z_{j}, z_{j}, t_{i}\right) \Delta z_{\mathrm{res}} .
$$

By considering the summand in this expression we get access to the energy density for the density fluctuations. Studying how it changes in time between different pixel positions $z_{j}$ will then give information about the dynamics of the energy of density fluctuations in the system.

For the single quasicondensate, as explained in the main text, it is not possible to measure directly the phase fluctuations. This is important, however, in order to assess the energy contribution coming from the gradient of the phase operator. This information can be obtained via a tomographic approach by studying the velocities of wave packets going through the system, as demonstrated recently [17]. The basic idea is that the phononic Hamiltonian can be put to a normal form

$$
\hat{H}=\sum_{k>0} \frac{\hbar \omega_{k}}{2}\left(\hat{\varphi}_{k}^{2}+\delta \hat{\varrho}_{k}^{2}\right)+\frac{g}{2} \delta \varrho_{0}^{2}
$$

using the eigenmode operators $\hat{\varphi}_{k}, \delta \hat{\varrho}_{k}$ that depend on the GP profile arising from cosine eigenfunctions in the homogeneous case. We then find that the dynamics of the density fluctuation operator reveals information about the phase operator by means of the relation

$$
\delta \hat{\varrho}_{k}(t)=\cos \left(\omega_{k} t\right) \delta \hat{\varrho}_{k}+\sin \left(\omega_{k} t\right) \hat{\varphi}_{k} .
$$

Exploiting this expression to relate observables at different times and using the analysis and reconstruction methods developed in Ref. [17] should then give access to the second moments of the phase fluctuations. Specifically, one would reconstruct the second moments $C_{k, k^{\prime}}^{\phi \phi}=\left\langle\hat{\varphi}_{k} \hat{\varphi}_{k^{\prime}}\right\rangle$ of eigenmodes $k, k^{\prime}$ and we can obtain the total energy 
contained in the phase sector by simply summing

$$
E_{\phi}(t)=\frac{1}{2} \sum_{k>0} \hbar \omega_{k} C_{k, k}^{\phi \phi}(t)
$$

Additionally, one can translate the second moments of the eigenmodes to real space after performing the derivative on the eigenfunctions, which should give the local information about the energy.

When considering two condensates one has access to interferometric measurements of the relative phase fluctuations $\hat{\varphi}_{\text {rel }}=\hat{\varphi}_{1}-\hat{\varphi}_{2}$ between two quasicondensates and based on nonequilibrium variations of that observable, relative density fluctuations $\delta \hat{\varrho}_{\text {rel }}=\delta \hat{\varrho}_{1}-\delta \hat{\varrho}_{2}$ were reconstructed in Ref. [17]. Density absorption is still available to measure density fluctuations of the common degrees of freedom $\delta \hat{\varrho}_{\text {com }}=\delta \hat{\varrho}_{1}+\delta \hat{\varrho}_{2}$ but is usually less revealing.

\section{APPENDIX B: GAUSSIAN MODELS IN THE SIMULATIONS OF QTPS}

The continuous Hamiltonian given in Eq. (5) can be appropriately discretized, which we explain in Appendix C1. The system can then be described in terms of quadrature operators, in particular, one can describe the quantum states and dynamics with the Gaussian framework of covariance matrices and symplectic transformations. In this section, we present a short summary of the formalism of Gaussian quantum information, see, e.g., Refs. [161,162] for more complete reviews on the subject.

We consider bosonic systems of $N$ bosonic modes, associated with quadratures

$$
\hat{\mathbf{X}}:=\left(\hat{q}_{1}, \hat{q}_{2}, \ldots, \hat{q}_{N}, \hat{p}_{1}, \hat{p}_{2}, \ldots, \hat{p}_{N}\right)^{T}
$$

that can be seen as the $N$ position and momentum operators, respectively. The canonical commutation relations can be captured as $\left[\hat{X}_{l}, \hat{X}_{m}\right]=i \Omega_{l, m}$ for $l, m=1, \ldots, N$, giving rise to the symplectic form

$$
\Omega=\left(\begin{array}{cc}
0 & \mathbb{1} \\
-\mathbb{1} & 0
\end{array}\right)
$$

Given a density matrix $\hat{\gamma}$, we define the vector of mean values $\overline{\mathbf{X}}:=\langle\hat{\mathbf{X}}\rangle_{\hat{\gamma}}=\operatorname{Tr}(\hat{\gamma} \hat{\mathbf{X}})$ : these are the first moments of the set of quadrature operators $\hat{X}$ corresponding to the quantum state. The second moments can be collected in the covariance matrix with entries

$$
\Gamma_{i, j}:=\left\langle\hat{X}_{i} \hat{X}_{j}+\hat{X}_{j} \hat{X}_{i}\right\rangle_{\hat{\gamma}}-2\left\langle\hat{X}_{i}\right\rangle_{\hat{\gamma}}\left\langle\hat{X}_{j}\right\rangle_{\hat{\gamma}}
$$

For a single mode, namely $N=1$, the diagonal elements of $\Gamma$ are simply the two variances $\Gamma_{1,1}=2\left(\Delta \hat{q}_{1}\right)_{\hat{\gamma}}^{2}$ and $\Gamma_{2,2}=$ $2\left(\Delta \hat{p}_{1}\right)_{\hat{\gamma}}^{2}$. The single constraint for the real-valued matrix to correspond to a physical state is given by the Heisenberg uncertainty relation, which can be concisely written as a semidefinite constraint as

$$
\Gamma+i \Omega \geq 0 .
$$

Of key importance in this work are bosonic Gaussian states. A general Gaussian state of $N$ modes is fully described by the vector of mean values and the covariance matrix corresponding to all modes. Gaussian states are ubiquitous in physical systems. For example, thermal states $\hat{\gamma}_{\beta}[\hat{H}]=\exp (-\beta \hat{H}) / \operatorname{Tr}[\exp (-\beta \hat{H})]$ are Gaussian whenever the Hamiltonian $\hat{H}$ is quadratic in the field operators, which again is a very common situation in many physical settings. In condensed-matter physics and in quantum field theory, such a situation would be referred to as being noninteracting. Generally, every Gaussian state with full support [163] can be written in a form resembling thermal states of quadratic Hamiltonians, namely there exists a $H$ such that

$$
\begin{aligned}
\hat{\gamma}[H] & =\frac{1}{Z} \exp \left(-\frac{1}{2}(\hat{\mathbf{X}}-\overline{\mathbf{X}})^{T} H(\hat{\mathbf{X}}-\overline{\mathbf{X}})\right), \\
H & =\left(\begin{array}{ll}
H_{q q} & H_{q p} \\
H_{p q} & H_{p p}
\end{array}\right),
\end{aligned}
$$

where $H$ is a real positive semidefinite $2 N \times 2 N$ matrix written in block form for clarity and

$$
\begin{aligned}
Z & =\operatorname{Tr}\left[\exp \left(-\frac{1}{2}(\hat{\mathbf{X}}-\overline{\mathbf{X}})^{T} H(\hat{\mathbf{X}}-\overline{\mathbf{X}})\right)\right] \\
& =\sqrt{\operatorname{det}[(\Gamma+i \Omega) / 2]}
\end{aligned}
$$

is the normalization, which can be fully determined by the covariance matrix of the Gaussian state $\Gamma$. The relation between $\Gamma$ and the matrix $H$ appearing in the expression above is

$$
H=2 i \Omega \operatorname{arcoth}(i \Gamma \Omega), \quad \Gamma=i \Omega \operatorname{coth}(i \Omega H / 2) .
$$

In turn, any generic quadratic (Hermitian) Hamiltonian can be written similarly as above, i.e., with $H$ being a real positive-semidefinite $2 N \times 2 N$ matrix. Thus, as a difference with respect to the above matrix appearing in the expression for faithful Gaussian states, a generic quadratic Hamiltonian can also contain zero eigenvalues (and need not to be diagonalizable).

The (Gaussian) unitary evolution corresponding to the time-independent quadratic Hamiltonian translates into the symplectic transformation acting on the covariance matrix, given by

$$
G(t)=\exp (\Omega H t)
$$

such that the evolved covariance matrix is $\Gamma(t)=$ $G(t) \Gamma(0) G(t)^{T}$. A similar relation holds for the evolution 
with time-dependent Hamiltonians, see, for example, the discussion on the QTP primitives in Appendix C. Thus, in the framework of Gaussian states and operations one can work directly with just the mean vector and the covariance matrix, since they jointly contain all the information that characterizes the Gaussian state. In particular, given a quadratic Hamiltonian $\hat{H}=\sum_{k, l} H_{k, l} \hat{X}_{k} \hat{X}_{l}$, the average energy of a state $\hat{\gamma}$ can be easily computed as

$$
\begin{aligned}
E_{\gamma}=\operatorname{Tr}(\hat{H} \hat{\gamma}) & =\sum_{k, l} H_{k, l} \operatorname{Tr}\left(\hat{\gamma} \hat{X}_{k} \hat{X}_{l}\right) \\
& =\sum_{k, l} H_{k, l}\left(\frac{1}{2} \Gamma_{k, l}+\left\langle X_{l}\right\rangle_{\gamma}\left\langle X_{k}\right\rangle_{\gamma}\right)
\end{aligned}
$$

The covariance matrix and the Hamiltonian matrix can be put into normal form by symplectic transformations, which read

$$
\Gamma=M\left(\bigoplus_{k} \gamma_{k} \mathbb{1}_{2}\right) M^{T}, \quad H=M\left(\bigoplus_{k} \omega_{k} \mathbb{1}_{2}\right) M^{T},
$$

where $M$ is a symplectic matrix and the $\left\{\gamma_{k}\right\}$ (respectively $\left.\left\{\omega_{k}\right\}\right)$ are the symplectic eigenvalues and are the eigenvalues of $|i \Omega \Gamma|$ (respectively $|i \Omega H|)$. Clearly, the symplectic eigenvalues of $\Gamma$ and $H$ are related to each other in the same relation as Eq. (B7), e.g., for a thermal covariance matrix at inverse temperature $\beta^{-1}=k_{B} T$, we have

$$
d_{k}=\operatorname{coth}\left(\beta \omega_{k} / 2\right)
$$

which is the usual relation between the normal-mode frequencies $\omega_{k}$ of a harmonic oscillator Hamiltonian and the normal covariances of its thermal state. Note that by identifying $\gamma_{k}=2\left\langle n_{k}\right\rangle+1$, this agrees with the Bose-Einstein number distribution formula

$$
\left\langle\hat{n}_{k}\right\rangle=e^{-\beta \omega_{k}} /\left(1-e^{-\beta \omega_{k}}\right) .
$$

The von Neumann entropy of a quantum state $\rho$ can be also directly computed from its covariance matrix $\Gamma$, and in particular just from its symplectic eigenvalues (as is true for every unitarily invariant quantity). In fact, recall the definition

$$
S(\hat{\gamma}):=-\operatorname{Tr}(\hat{\gamma} \log \hat{\gamma}),
$$

and that it is invariant under unitaries. By considering the density matrix expressed as in Eq. (B5), we notice that we can first apply local unitaries (namely displacement operators) so to put $\overline{\mathbf{X}}_{\rho}=0$. Then, by taking the matrix logarithm, we find the expression for the von Neumann entropy of a (faithful) Gaussian state to be

$$
\begin{aligned}
S(\hat{\gamma}[H])= & \frac{1}{2} \log \operatorname{det}\left(\frac{\Gamma+i \Omega}{2}\right) \\
& +\frac{1}{2} \sum_{k, l} \operatorname{arcoth}(i \Gamma \Omega)_{k, l}(i \Omega \Gamma)_{l, k},
\end{aligned}
$$

and in terms the symplectic eigenvalues of the covariance matrix it reads

$$
\begin{aligned}
S(\hat{\gamma}[H])= & \sum_{k=1}^{N}\left[\left(\frac{d_{k}+1}{2}\right) \log \left(\frac{d_{k}+1}{2}\right)\right. \\
& \left.-\left(\frac{d_{k}-1}{2}\right) \log \left(\frac{d_{k}-1}{2}\right)\right] .
\end{aligned}
$$

For a thermal covariance matrix with $\beta>0$, we can rewrite this expression in terms of normal mode frequencies:

$$
S(\beta)=\sum_{k}\left[\frac{\beta \omega_{k} e^{-\beta \omega_{k}}}{1-e^{-\beta \omega_{k}}}-\log \left(1-e^{-\beta \omega_{k}}\right)\right] .
$$

Recall that $F(\cdot)=\operatorname{Tr}(H \cdot)-S(\cdot) / \beta$ is the nonequilibrium free energy of the state relative to its surrounding ambient temperature $\beta^{-1}$ and its corresponding Hamiltonian $H$. In the case of thermal states, the free energy is given as

$$
\begin{aligned}
F(\beta) & =\operatorname{Tr}\left(\hat{H} \hat{\gamma}_{\beta}[\hat{H}]\right)-\beta^{-1} S(\beta) \\
& =\beta^{-1} \sum_{k} \log \left(1-e^{-\beta \omega_{k}}\right) .
\end{aligned}
$$

Given two faithful Gaussian states $\hat{\gamma}$ and $\hat{\sigma}$, each on $N$ bosonic modes, described by covariance matrices $\Gamma$ and $\Upsilon$, respectively, it is also easy to compute their relative entropy according to

$S(\hat{\gamma} \| \hat{\sigma})=-S(\hat{\gamma})-\operatorname{Tr}(\hat{\gamma} \log \hat{\sigma})=\operatorname{Tr}[\hat{\gamma}(\log \hat{\gamma}-\log \hat{\sigma})]$,

essentially because again it is easy to compute the logarithm of such states. Since the first term is nothing but the negative von Neumann entropy that can be computed according to Eq. (B15), we can see this by just considering the second term. By considering the form, Eq. (B5), of faithful Gaussian states, we obtain

$$
\begin{aligned}
& \operatorname{Tr}(\hat{\gamma} \log \hat{\sigma}) \\
& \quad=-\log Z_{\sigma}+\operatorname{Tr}\left[\hat{\gamma}\left(-\frac{1}{2}\left(\hat{\mathbf{X}}-\overline{\mathbf{X}}_{\sigma}\right)^{T} H_{\sigma}\left(\hat{\mathbf{X}}-\overline{\mathbf{X}}_{\sigma}\right)\right)\right]
\end{aligned}
$$


where we simply use the fact that the logarithm and exponential of a matrix are inverse functions. We can also simplify further the above expression and write it just in terms of (combinations of) covariance matrices elements as

$$
-\operatorname{Tr}(\hat{\gamma} \log \hat{\sigma})=\frac{1}{2} \log \operatorname{det}[(\Upsilon+i \Omega) / 2]+\frac{1}{4} \sum_{k, l} \Upsilon_{k, l}\left(H_{\sigma}\right)_{k, l}+\frac{1}{2}\left(\overline{\mathbf{X}}_{\sigma}-\overline{\mathbf{X}}_{\rho}\right)^{T} H_{\sigma}\left(\overline{\mathbf{X}}_{\sigma}-\overline{\mathbf{X}}_{\rho}\right),
$$

which leads to

$$
S(\hat{\gamma} \| \hat{\sigma})=\frac{1}{2}\left[\log \left(\frac{\operatorname{det}[(\Upsilon+i \Omega) / 2]}{\operatorname{det}[(\Gamma+i \Omega) / 2]}\right)+\frac{1}{2} \sum_{k, l} \Gamma_{k, l}\left(H_{\sigma}-H_{\rho}\right)_{k, l}+\left(\overline{\mathbf{X}}_{\sigma}-\overline{\mathbf{X}}_{\rho}\right)^{T} H_{\sigma}\left(\overline{\mathbf{X}}_{\sigma}-\overline{\mathbf{X}}_{\rho}\right)\right],
$$

where we also use the expression (B14) for the von Neumann entropy. Note once more that the matrices $H_{\rho}$ and $H_{\sigma}$ can be also directly obtained from $\Gamma$ and $\Upsilon$, respectively, through Eq. (B7). Another useful expression can be written down, containing explicitly the symplectic eigenvalues of the two covariance matrices. For that we notice that the logarithm of the partition function $Z_{\sigma}$ can also be expressed as

$$
\log Z_{\sigma}=\frac{1}{2} \sum_{k} \log \left[\left(v_{k}^{2}-1\right) / 2\right]
$$

where $\left\{v_{k}\right\}$ are the symplectic eigenvalues of $\Upsilon$. Thus, we can write

$$
\begin{aligned}
S(\hat{\gamma} \| \hat{\sigma})= & -S(\hat{\gamma})+\sum_{k} \log \left[\left(v_{k}^{2}-1\right) / 2\right] \\
& +\frac{1}{4} \sum_{k, l} \Gamma_{k, l}\left(H_{\sigma}\right)_{k, l} \\
& +\frac{1}{2}\left(\overline{\mathbf{X}}_{\sigma}-\overline{\mathbf{X}}_{\rho}\right)^{T} H_{\sigma}\left(\overline{\mathbf{X}}_{\sigma}-\overline{\mathbf{X}}_{\rho}\right),
\end{aligned}
$$

where we can also use the expression (B15) for $S(\rho)$. Finally, note that if $\sigma$ is a true thermal state of a Hamiltonian $H$ at inverse temperature $\beta>0$, then for any state $\rho$, we have

$$
S(\hat{\gamma} \| \hat{\sigma})=\beta[F(\hat{\gamma})-F(\hat{\sigma})]
$$

\section{APPENDIX C: DETAILS OF THE QTP SIMULATIONS}

\section{Lattice discretization scheme}

Here we define a lattice version of the phononic Hamiltonian, obtained by discretizing the interval $[-L, L]$ into $N$ pixels, each of size $\Delta z=2 L / N[21,164]$. This is particularly important to make numerical calculations, especially for the case of nonhomogeneous external potentials. Fixing $N$, for $i=1, \ldots, N+1$ the coordinates of the discretization lattice read $z_{i}=-L+2 L \frac{i-1}{N}$, and we define discretization pixels, which are the closed intervals $p_{i}=\left[z_{i}, z_{i+1}\right]$ for $i=1, \ldots, N$. We then introduce the discretized version of density and phase operators as the integration of the field operators via

$$
\hat{\varphi}_{i}^{(N)}=\frac{1}{\Delta z} \int_{p_{i}} d z \hat{\varphi}(z), \quad \delta \hat{\varrho}_{i}^{(N)}=\frac{1}{\Delta z} \int_{p_{i}} d z \delta \hat{\varrho}(z),
$$

with $\left|p_{i}\right|=\Delta z=2 L / N$. These discretized operators yields a vector of canonical coordinates

$$
\hat{\mathbf{X}}=\left(\delta \hat{\varrho}_{1}^{(N)} \ldots \delta \hat{\varrho}_{N}^{(N)}, \hat{\varphi}_{1}^{(N)}, \ldots \hat{\varphi}_{N}^{(N)}\right)^{T},
$$

satisfying (rescaled) bosonic canonical commutation relations

$$
\left[\hat{X}_{j}, \hat{X}_{k}\right]=i \Omega_{j, k} / \Delta z
$$

where $\Omega$ is defined in Eq. (B2). As explained in Ref. [17], in the continuum limit $N \rightarrow \infty$, the right-hand side will yield a Dirac delta because $1 / \Delta z$.

To discretize the model, we follow Ref. [21] and consider the geometric mean

$$
\eta_{i}=\sqrt{\rho_{0}\left(z_{i}\right) \rho_{0}\left(z_{i+1}\right)}
$$

for $i=1, \ldots, N$. The discretization of the effective model will be a quadratic operator in the discretized modes $\hat{\varphi}_{i}^{(N)}$ and $\delta \hat{\varrho}_{i}^{(N)}$. At the lowest-order approximation, one obtains

$$
\begin{aligned}
\hat{H} \approx & \Delta z \sum_{i=1}^{N-1} \frac{\hbar^{2} \eta_{i}}{2 m}\left[\frac{\hat{\varphi}_{i}^{(N)}-\hat{\varphi}_{i+1}^{(N)}}{\Delta z}\right]^{2} \\
& +\Delta z \sum_{i=1}^{N} \frac{g\left(z_{i}\right)}{2}\left(\delta \hat{\varrho}_{i}^{(N)}\right)^{2}=: \hat{H}_{N} .
\end{aligned}
$$

Note that so far in the main text, we have suppressed for simplicity the possible spatial dependence of the coupling 
constant $g$, which is true for a homogeneous quasicondensate and in general has little influence. In general, $g$ depends on the GP profile,

$$
g(z)=\hbar \omega_{\perp} a_{s}\left[2+3 a_{s} \rho_{0}(z)\right] /\left[1+2 a_{s} \rho_{0}(z)\right]^{3 / 2},
$$

where $\omega_{\perp}$ is the radial trapping frequency and $a_{s}$ is the scattering length $[33,165]$. This dependency on the spatial coordinate $z$ has been included in our numerical simulations. From this, we obtain the matrix representation of the above Hamiltonian

$$
\begin{aligned}
H & =\frac{1}{2} \cdot \hat{\mathbf{X}}^{\top}\left(H_{\rho \rho}[g, \Delta z] \oplus H_{\phi \phi}\left[\rho_{0}, \Delta z\right]\right) \hat{\mathbf{X}} \\
H_{\rho \rho}[\Delta z] & =\Delta z \cdot \operatorname{diag}\left[g\left(z_{1}\right), g\left(z_{2}\right), \ldots, g\left(z_{N_{c}}\right)\right] \\
H_{\phi \phi}\left[\rho_{0}, \Delta z\right] & =\frac{\hbar^{2}}{m \Delta z}\left(\begin{array}{cccc}
\eta_{1} & -\eta_{1} & \\
-\eta_{1} & \eta_{1}+\eta_{2} & -\eta_{2} \\
& \ddots & \\
& -\eta_{N-2} & \eta_{N-2}+\eta_{N-1} & -\eta_{N-1} \\
& & -\eta_{N-1} & \eta_{N-1}
\end{array}\right)+2 \hbar \operatorname{diag}\left[J\left(z_{1}\right) \eta\left(z_{1}\right), \ldots, J\left(z_{N}\right) \eta\left(z_{N}\right)\right],
\end{aligned}
$$

where we have used the functional notation $H_{\phi \phi}\left[\rho_{0}, \Delta z\right]$ to emphasize that these couplings depend on the mean-field density profile and the size of the pixels. We additionally added a small term $\propto J$, which is meant to regularize the zero mode. This way, all computations are made with fully supported Gaussian states so that numerical instabilities do not occur. Physically, it can be interpreted as adding a small mass term of the type $\hat{H}_{J}=h J \int \mathrm{d} z \rho_{0}(z) \hat{\varphi}(z)^{2}$ and we have checked that, as long as the coupling is chosen to be around $J \approx 0.01$ the dynamics is not affected in the times scales of $300 \mathrm{~ms}$ that we have in mind. See also Appendix C3 below for a more extended discussion.

Starting from a set of canonical coordinates $\hat{\mathbf{X}}$, then for a symplectic $M \in \mathbb{R}^{2 N \times 2 N}$, i.e., fulfilling

$$
M \Omega M^{T}=\Omega,
$$

we have that $\hat{\mathbf{r}}=M \hat{\mathbf{X}}$ will again denote a vector of canonically commuting operators, which can be seen by explicitly checking that $\hat{r}$ again fulfills $\left[\hat{r}_{j}, \hat{r}_{k}\right]=i \Omega_{j, k} / \Delta z$.

We can then diagonalize our Hamiltonian as follows: first, we use the symplectic matrix

$$
M_{1}=\left(\begin{array}{cc}
\sqrt{H_{\rho \rho}^{-1}} & 0 \\
0 & \sqrt{H_{\rho \rho}}
\end{array}\right)=M_{1}^{T},
$$

since $H_{\rho \rho}$ is diagonal. Then, we have

$$
M_{1}^{T} H M_{1}=\mathbb{1}_{N} \oplus\left(\sqrt{H_{\rho \rho}^{-1}} H_{\phi \phi} \sqrt{H_{\rho \rho}}\right)=: \mathbb{1}_{N} \oplus \tilde{H}_{\phi \phi},
$$

where $\tilde{H}_{\phi \phi}$ is the matrix of the phase couplings in the new coordinates, which is real and symmetric, and therefore can be diagonalized by an orthogonal transformation $O$ with $\tilde{H}_{\phi}=O \Sigma O^{T}$. Here, $\Sigma$ is diagonal and we assume that all zero eigenvalues are sorted to the first $N^{0} \geq 0$ positions, i.e., $\Sigma=0_{N^{0}} \oplus \tilde{\Sigma}$ with $\tilde{\Sigma}>0$ diagonal and we define the eigenfrequencies $\omega$ via $\tilde{\Sigma}^{1 / 2}=\operatorname{diag}\left(\omega_{N^{0}+1}, \ldots, \omega_{N}\right)$. With the diagonal matrix $\Sigma_{\phi}=\mathbb{1}_{N^{0}} \oplus \tilde{\Sigma}$ and the transformation

$$
M_{2}=\left(\begin{array}{cc}
O \Sigma_{\phi}^{1 / 4} & 0 \\
0 & O \Sigma_{\phi}^{-1 / 4}
\end{array}\right)
$$

we obtain

$$
M_{2}^{T} M_{1}^{T} H M_{1} M_{2}=\left(\mathbb{1}_{N^{0}} \oplus \tilde{\Sigma}^{1 / 2}\right) \oplus\left(0_{N^{0}} \oplus \tilde{\Sigma}^{1 / 2}\right) .
$$

That is, in the canonical coordinates $\hat{\mathbf{r}}=\left(\hat{Q}_{1}, \ldots, \hat{Q}_{N}\right.$, $\left.\hat{P}_{1}, \ldots, \hat{P}_{N}\right)=\sqrt{\Delta z}\left(M_{1} M_{2}\right)^{-1} \hat{\mathbf{X}}$ we have that the Hamiltonian in Eq. (C7) takes the form

$$
\hat{H}=\frac{1}{2} \sum_{j=1}^{N^{0}} \hat{Q}_{j}^{2}+\frac{1}{2} \sum_{j=N^{0}+1}^{N} \omega_{j}\left(\hat{P}_{j}^{2}+\hat{Q}_{j}^{2}\right) .
$$

Finally, we can define creation and annihilation operators $\left(\hat{c}_{j}^{\dagger}, \hat{c}_{j}\right)$ for each normal mode from the relation

$$
\hat{P}_{j}^{2}+\hat{Q}_{j}^{2}=2 \hat{c}_{j}^{\dagger} \hat{c}_{j}+\hat{\mathbb{1}}
$$

Note that the new coordinates satisfy true canonical commutation relations $\left[\hat{Q}_{k}, \hat{P}_{l}\right]=i \delta_{k, l}$ and consequently we 
also have

$$
\left[\hat{c}_{k}, \hat{c}_{l}^{\dagger}\right]=\delta_{k, l}
$$

for all $k, l$. However, our original discretized field operators satisfy rescaled commutation relations. This means that the symplectic matrix corresponding to the evolution with $\hat{H}_{N}$ in the original coordinates is given by Eq. (B8), where the symplectic form is rescaled, namely $\Omega \mapsto(\Delta z)^{-1} \Omega$.

Thermal states of the above Hamiltonian have covariance matrices of the form $\Gamma=\Gamma_{\rho \rho} \oplus \Gamma_{\phi \phi}$, and can also be explicitly computed from the normal modes and the corresponding symplectic transformation, namely Eqs. (B10) and (B11). The expression is somewhat complicated for the general case, but for the special case of homogeneous systems (which we are interested in) $H_{\rho \rho}=\kappa \mathbb{1}$ with $\kappa:=\Delta z g$, we get

$$
\Gamma_{\beta}=\frac{1}{\sqrt{\kappa}} H_{\phi \phi}^{1 / 2} \oplus \sqrt{\kappa} H_{\phi \phi}^{-1 / 2}+\frac{1}{\sqrt{\kappa}}\left(H_{\phi \phi}^{1 / 2} T\right) \oplus \sqrt{\kappa}\left(H_{\phi \phi}^{-1 / 2} T\right),
$$

where

$$
T:=2\left(\exp \left(2 \beta \sqrt{\kappa} H_{\phi \phi}^{1 / 2}\right)-\mathbb{1}\right)^{-1}
$$

As discussed in the previous Appendix B, diagonalizing the Hamiltonian in terms of normal modes, the covariance matrix becomes also diagonal with symplectic eigenvalues given by Eq. (B11). From these symplectic eigenvalues one can also write down the (von Neumann) entropy and the free energy as in Eqs. (B16) and (B17).

\section{Details of merging and splitting}

In this section, we provide an extended discussion of the merge and split primitive. This is a three-step process, involving two condensates $A$ and $B$ with lengths $L_{A}$ and $L_{B}$ and densities $\rho_{0}^{A}$ and $\rho_{0}^{B}$, and consisting in the following:

(a) merging the two initially independent condensates during a time $t_{\text {merge }}$;

(b) letting them evolve with the fully merged Hamiltonian for a time $t_{\text {evolve }}$; and

(c) splitting the joint condensate back into two parts $A$ and $B$, with the same lengths as the initials, during a time $t_{\text {split }}$.

\section{a. Merging}

For the merging process, we encounter a time-dependent Hamiltonian $\hat{H}_{A-B}(t)$ such that

$$
\hat{H}_{A-B}(0)=\hat{H}_{N^{A}}\left[\rho_{0}^{A}\right]+\hat{H}_{N^{B}}\left[\rho_{0}^{B}\right],
$$

where our Hamiltonians are given by the lattice model in Eq. (C5) and (keeping constant the small distance cutoff
$\Delta z$ ) are functionals of the initial mean-field density profiles of the two condensates. Note that since we would like to couple the two systems, we require them to have a consistent momentum cutoff $(\Delta z)^{A}=(\Delta z)^{B}=\Delta z$ (so that waves traveling across quasicondensates with same atom density in the simulation should not change in speed due to the different discretization), and consequently their number of pixels will be in the same proportion as their lengths, i.e.,

$$
N^{A}=L_{A} \Delta z=N^{B} L_{A} / L_{B}
$$

The coupling matrix of the uncoupled Hamiltonian in Eq. (C20) is by

$$
H_{\rho \rho, A \mid B}=H_{\rho \rho, A} \oplus H_{\rho \rho, B}, \quad H_{\phi \phi, A \mid B}=H_{\phi \phi, A} \oplus H_{\phi \phi, B} .
$$

To merge the condensates, an interaction Hamiltonian is switched on, so that the joint Hamiltonian as in Eq. (C7) has a matrix representation given by

$$
\begin{aligned}
& H_{\rho \rho, A B}=H_{\rho \rho, A} \oplus H_{\rho \rho, B}, \\
& H_{\phi \phi, A B}=H_{\phi \phi, A} \oplus H_{\phi \phi, B}+\frac{t}{t_{\text {merge }}} H_{\text {int }},
\end{aligned}
$$

where the interaction matrix is given by

$$
\begin{aligned}
\left(H_{\mathrm{int}}\right)_{i, j}= & \frac{\hbar^{2}}{2 m \Delta z} \eta_{N^{A}}\left(\delta_{N^{A}, i} \delta_{N^{A}, j}+\delta_{N^{A}+1, i} \delta_{N^{A}+1, j}\right. \\
& \left.-\delta_{N^{A}, i} \delta_{N^{A}+1, j}-\delta_{N^{A}+1, i} \delta_{N^{A}, j}\right)
\end{aligned}
$$

with $\eta_{N^{A}}:=\sqrt{\rho^{A}\left(N^{A}\right) \cdot \rho^{B}(1)}$. Note that this interaction contains also the local terms in the boundary region $\left[N^{A}, N^{A}+1\right]$. We hence see that the couplings during the merging are given by

$$
H_{A-B}(t)=\left(1-\frac{t}{t_{\text {merge }}}\right) H_{A \mid B}+\frac{t}{t_{\text {merge }}} H_{A B} .
$$

For the numerical implementation, we also discretize the time evolution so that we divide the $\left[0, t_{\text {merge }}\right]$ time interval into $N_{t}$ steps of duration $\Delta t=t_{\text {merge }} / N_{t}$. Then, the symplectic evolution matrix reads

$$
G_{\text {merge }}\left(t_{\text {merge }}\right)=\prod_{j=1}^{N_{t}} \exp \left[\Omega H_{A-B}\left(t_{j}\right) / \Delta z\right],
$$

where $H_{A-B}(t)=H_{N^{A}}+H_{N^{B}}+\left(j / N_{t}\right) H_{\text {int }}$. Examples of eigenmodes for the time-dependent Hamiltonian are plotted in Figs. 8 and 9 for a homogeneous GP profile with or without a trapezelike buffer region. We find that mode functions that are odd in $z$ hybridize via a jump, which 

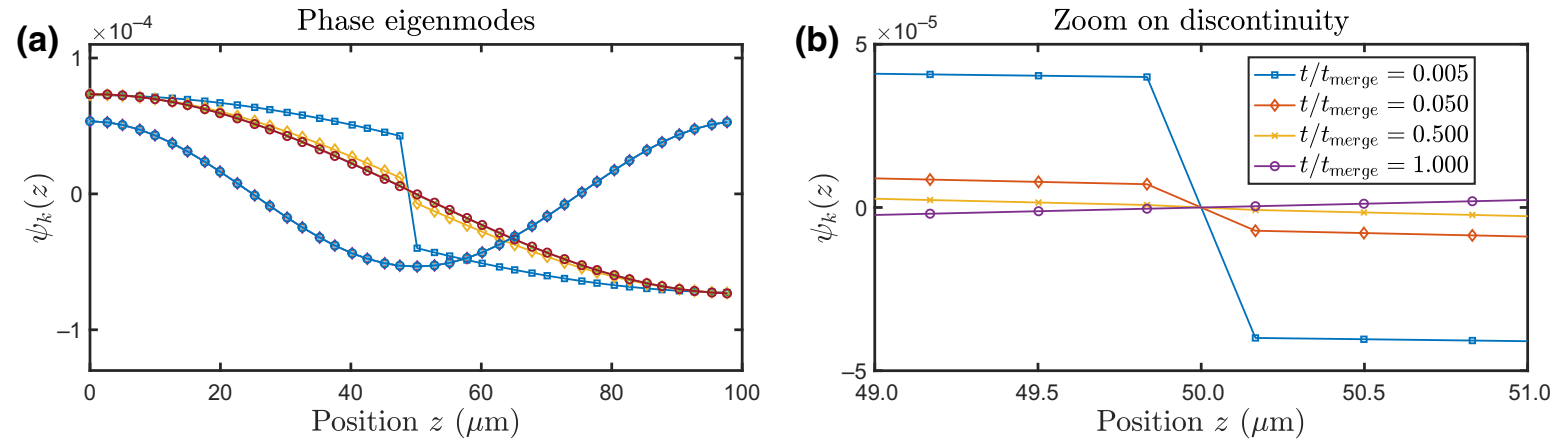

FIG. 8. Snapshots of the phase and density eigenmode functions for the first and second lowest modes, taken at different Trotter steps $t / t_{\text {merge }}$ during merging for a fully homogeneous profile.

gets smoothened during the merging while, mode functions, which are even in $z$ get glued automatically. We see also that all modes have a local extremum at the boundary, which means we have Neumann boundary conditions.

In the main text we have shown the results for a model of the quasicondensates where the GP profile falls off smoothly from its peak value in the bulk to a lower value on the edges. In principle, it is possible to consider the effective model to be constant everywhere, whereas the edge of the condensate (where excitations get reflected) can be modeled by the boundary conditions. However, this abstraction turns out to be too simplistic. Figure 10 provides a demonstration of what occurs in such a scenario. Since the process is simulated via the merging of the boundary conditions of the two condensates, in particular occurring at a single pixel, it is hence independent of the momentum cutoff. As a result, momenta at all scales are populated, however, this does not faithfully capture the physics of the continuum model, since the dispersion relation is not linear. In order to avoid this, it is therefore necessary for the model to resolve details of the coupling zone.

\section{b. Idle evolution}

In between merging and splitting, one can allow some idle evolution time $t_{\text {evolve }}$ in which the joint system evolves with the fully coupled Hamiltonian. This can be applied with a single symplectic matrix, since the Hamiltonian is time independent. Wave packets injected during the previous merging process will travel ballistically through the entire joint system (as long as we have taken care to remain in the regime where high-momentum modes are negligible and the linear dispersive relation holds).

\section{c. Splitting}

Finally, we implement the splitting procedure by a timedependent Hamiltonian reversing the linear interpolation that has been discussed for merging. In the numerical simulation the covariance matrix of the $A$ and $B$ quasicondensates after merging, idle evolution, and splitting would have the form

$$
\Gamma_{A-B}\left(t_{\text {tot }}\right)=G_{\text {split }} G_{\text {evolve }} G_{\text {merge }} \Gamma_{A-B}(0) G_{\text {merge }}^{T} G_{\text {evolve }}^{T} G_{\text {split }}^{T}
$$

(a)

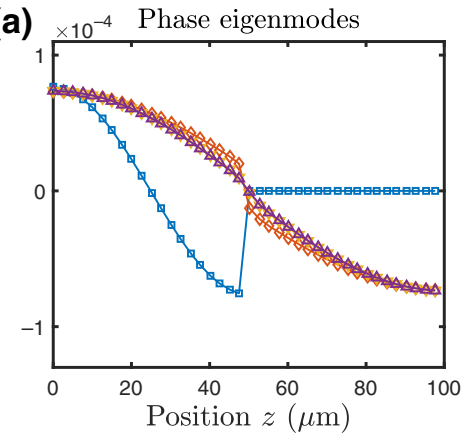

(b)

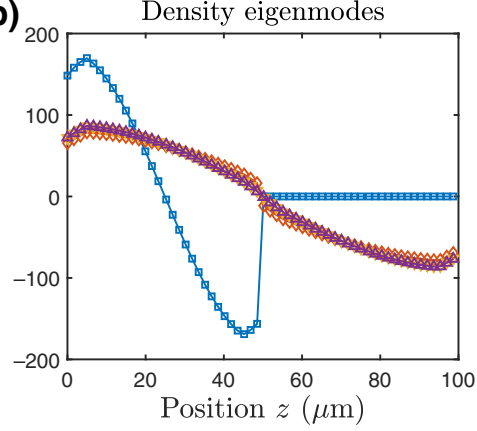

(c)

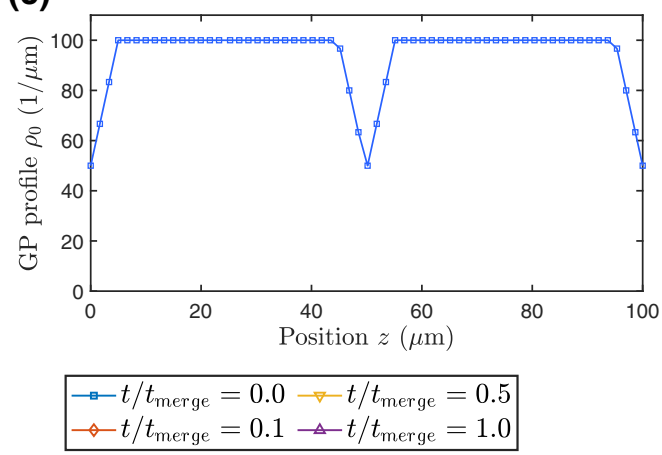

FIG. 9. Snapshots of the phase and density eigenmode functions for the two lowest-lying modes taken at different Trotter steps $t / t_{\text {merge }}$ during merging for a homogeneous profile with a trapezelike buffer region. (a) The odd modes during the coupling have a discontinuity of varying strength, which diminishes as the merging proceeds. In contrast, there is little influence of the merging on the even modes as they can be obtained by connecting the odd modes of the individual uncoupled systems. (b) The discontinuity is sharp, changing suddenly from one pixel to another. 
(a)

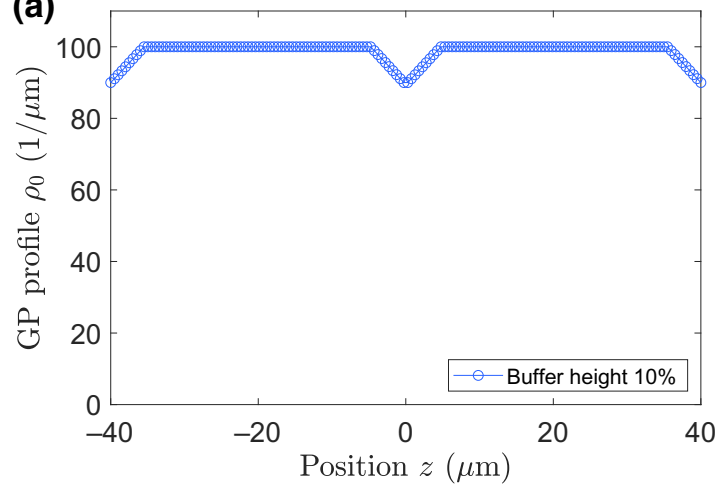

(b)

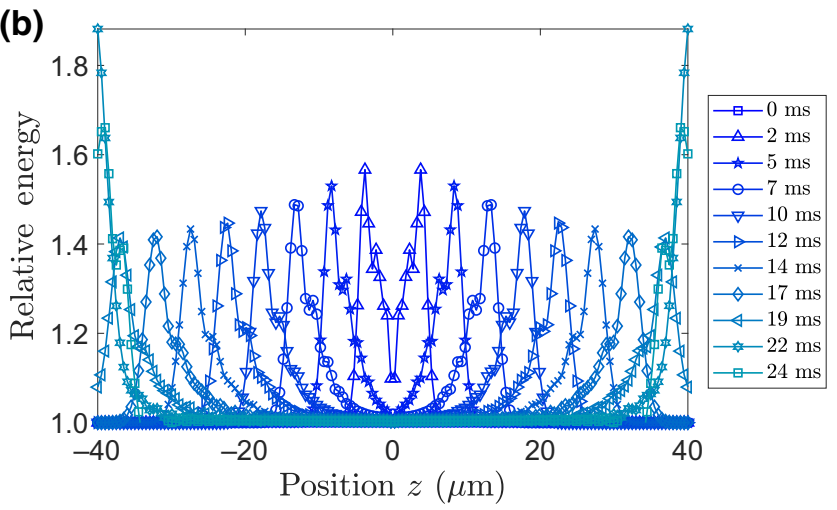

FIG. 10. The presence of high-momentum modes in a merging model with a nonextensive buffer region between condensates. (a) We show the merging scenario similar to the figure in the main text with the difference that the coupling zone is much smaller and ends more abruptly at $90 \%$ of the peak value. (b) We now find that the transport of the excitations is dispersive, which can be seen by the peaks of wave packets falling down as their propagation. As the dynamics is modeled to be unitary and the Hamiltonian does not change in the bulk this means that the energy the wave packets carry stays constant while being broadened. In lattice theories, dispersion can be proven analytically if short wavelengths are present in the state [166].

For reasons discussed in the main text, whenever we simulate the Otto cycle, we neglect the correlations between the two parts of the split condensate at the end of the process. In other words, we project the final covariance matrix into the direct sum of the two local covariance matrices for systems $A$ and $B$, i.e., at the end of the full protocol, $t=t_{\text {tot }}=t_{\text {merge }}+t_{\text {evolve }}+t_{\text {split }}$, by setting

$$
\Gamma_{A-B}^{\mathrm{fin}}\left(t_{\mathrm{tot}}\right)=\Gamma_{A}\left(t_{\mathrm{tot}}\right) \oplus \Gamma_{B}\left(t_{\mathrm{tot}}\right),
$$

where $\Gamma_{A}\left(t_{\mathrm{tot}}\right)$ is the submatrix of $\Gamma_{A-B}\left(t_{\mathrm{tot}}\right)$ corresponding to the subsystem $A$ and $\Gamma_{B}\left(t_{\text {tot }}\right)$ corresponds to $B$.

\section{d. Energy density injected during merging QTP}

Given the quadratic Hamiltonian over time $\hat{H}_{A-B}(t)$ we would like to study also the spatial distribution of the energy. In the discretized models, it is natural to study the energy per pixel $z$, namely

$$
E(z, t)=E_{z}(t) \Delta z=\frac{1}{2}\left[H_{A-B}(t) \Gamma_{A-B}(t)\right]_{z, z} \Delta z,
$$

where the notation $(\cdot)_{z, z}$ refers to the diagonal matrix element at pixel $z$. Note that if $\Delta z$ is constant, this amounts to just computing the quantities $E_{z}(t)$. Plotting $E_{z}$ over pixel positions is then a way of visualizing which regions in space have more energy than others. Doing this over varying times can show us how energy flows over time from one part of the system to the other. For example, in Fig. 10, we observe that merging the two systems amounts to inserting energy at their boundary continuously over the merging time (or in discrete bits at each Trotter time step). This energy then flows through the system at speed of sound velocity (which is $c=\sqrt{g \rho_{0} / m} \propto \sqrt{\rho_{0}}$ ), reaching the external boundaries and then bouncing back toward the center. Thus, in particular, if the ratio between the coupling time $t_{\text {merge }}$ and the length of a system (say $A$ ) is chosen such that

$$
c=L_{A} / t_{\text {merge }},
$$

then the energy perturbation precisely reaches the external boundary of system $A$. Similarly for system $B$. Clearly, then, when the two lengths $L_{A}$ and $L_{B}$ are not equal the energy flow cannot be synchronized so that the perturbation wave bounces back to the interface from both external walls at the same time. During the idle evolution time $t_{\text {evolve }}$ no additional energy is injected, but the energy flow continues. Finally, during the splitting process some energy is taken away from the system, again continuously over the splitting time and at the interface between the two parts. However, the total amount of energy taken back during the splitting is in general lower than the one inserted during merging. Hence, the total energy inserted during the entire protocol is always non-negative, and the amount is smaller given a protocol with longer time.

As a last comment, we note that the fact that our simulations use discretized space and time also implies that, besides the fact that energy is injected and ejected at the interface in discretized bits over $t_{\text {merge }}$ and $t_{\text {split }}$, the energy flow also takes places in pixels over time steps. In particular, all of this imposes us once more for consistency to make sure that the two coupled systems have the same small distance cutoff $\Delta z$, which also ensures that the lengths in the two systems are in the same ratios between their number of pixels. This issue becomes particularly important when a compression and expansion QTP takes place before merging since in that case, as we are going to discuss in detail in the next subsection, the cutoff $\Delta z$ changes in time. 


\section{Regularization of the zero mode: phase locking via excitation tunneling}

Here we discuss more in detail the additional complications arising from the zero modes of the phononic model and how to regularize them, in order to avoid instabilities during coupling. The mode expansion of Eq. (5) reads

$$
\hat{H}_{\mathrm{P}}\left[\rho_{0}\right]=\sum_{k>0} \hbar \frac{\omega_{k}}{2}\left(\hat{\varphi}_{k}^{2}+\delta \hat{\varrho}_{k}^{2}\right)+\frac{g}{2} \delta \hat{\varrho}_{\mathrm{ZM}}^{2},
$$

where $\omega_{k}$ are the eigenfrequencies of the phase and momentum eigenmodes $\hat{\varphi}_{k}, \delta \hat{\varrho}_{k}$ and there is a special mode, called the zero mode, $\delta \hat{\varrho}_{\mathrm{ZM}} \propto \int d z \delta \hat{\varrho}(z)$, which is different from $k>0$ eigenmodes as the canonically conjugate quantity $\hat{\varphi}_{\mathrm{ZM}} \propto \int d z \hat{\varphi}(z)$ does not appear in the Hamiltonian, i.e., it does not cost energy. The zero mode has the interpretation of total momentum frame of the excitations [29-32].

This mode expansion can be found in the continuum limit by solving the set of partial differential equation associated to the Heisenberg equations of motion, namely

$$
\left\{\begin{array}{c}
\partial_{t} \delta \varrho(z, t)=\frac{\hbar}{m} \partial_{z}\left[\rho_{0}(z) \partial_{z} \varphi(z, t)\right] \\
\partial_{t} \varphi(z, t)=-\frac{g}{\hbar} \delta \varrho(z, t) \\
\Rightarrow \partial_{t}^{2} \varphi_{k}(z, t)=-\frac{g}{m} \partial_{z}\left[\rho_{0}(z) \partial_{z} \varphi_{k}(z)\right],
\end{array}\right.
$$

and, as usual, for the $k>0$ modes we can look for solutions of the type $\varphi_{k}(z, t)=\varphi_{k}(z) e^{i \omega_{k} t}$, so that Eq. (C32) becomes a Sturm-Liouville problem

$$
\omega_{k}^{2} \varphi_{k}(z)=-\frac{g}{m} \partial_{z}\left[\rho_{0}(z) \partial_{z} \varphi_{k}(z)\right],
$$

and similarly for $\delta \varrho(z, t)$. We can then find solutions that form an orthonormal basis with respect to the scalar product

$$
\langle f, g\rangle:=\int d z f(z) g(z),
$$

i.e., we have $\left\langle\varphi_{k}(z), \varphi_{l}(z)\right\rangle=\left\langle\delta \varrho_{k}(z), \delta \varrho_{l}(z)\right\rangle=\delta_{k, l}$, where $\delta_{k, l}$ is the Kronecker delta.

However, besides those, one can also find a solution with $\omega_{k}=0$, which gives rise to the zero mode with quadrature operators denoted by $\left(\delta \hat{\varrho}_{\mathrm{ZM}}\right.$ and $\left.\hat{\varphi}_{\mathrm{ZM}}\right)$. These are necessary for the set of eigenmode functions to be complete and we can expand the field operators as

$$
\begin{aligned}
\delta \hat{\varrho}(z, t)= & \delta \hat{\varrho}_{\mathrm{ZM}}+\sum_{k>0} \sqrt{\frac{\hbar \omega_{k}}{g}} \delta \varrho_{k}(z)\left(e^{i \omega_{k} t} \hat{a}_{k}^{\dagger}+e^{-i \omega_{k} t} \hat{a}_{k}\right), \\
\hat{\varphi}(z, t)= & \hat{\varphi}_{\mathrm{ZM}}-\frac{g}{\hbar} t \delta \hat{\varrho}_{\mathrm{ZM}}-i \sum_{k>0} \sqrt{\frac{g}{\hbar \omega_{k}}} \varphi_{k}(z) \\
& \times\left(e^{i \omega_{k} t} \hat{a}_{k}^{\dagger}-e^{-i \omega_{k} t} \hat{a}_{k}\right),
\end{aligned}
$$

and we define eigenmode operators at $t=0$ (with $k>0$ ) from the relations

$$
\delta \hat{\varrho}_{k}=\sqrt{\frac{\hbar \omega_{k}}{g}}\left(\hat{a}_{k}^{\dagger}+\hat{a}_{k}\right), \quad \hat{\varphi}_{k}=-i \sqrt{\frac{g}{\hbar \omega_{k}}}\left(\hat{a}_{k}^{\dagger}-\hat{a}_{k}\right),
$$

such that they obey canonical commutation relations $\left[\delta \hat{\varrho}_{k}, \hat{\varphi}_{l}\right]=i \delta_{k, l}$ for all $k, l$.

Let us now consider the time evolution when coupling two systems governed by the Hamiltonian

$$
\hat{H}_{A-B}(t)=\left(1-\frac{t}{t_{\text {merge }}}\right) \hat{H}_{A \mid B}+\frac{t}{t_{\text {merge }}} \hat{H}_{A B}
$$

for $t \in\left[0, t_{\text {merge }}\right]$. Note that now at each instant $t$ this Hamiltonian has implicitly different boundary conditions at the interface $z=0$. See also Figs. 8 and 9 where the eigenmode functions of this time-dependent Hamiltonian are shown at different times $t / t_{\text {merge }}$ for the discretized model.

Thus, we see that, while coupling, the zero modes of the two systems will hybridize to form the joint zero mode and one mode that costs energy. However, this energy cost will cause the coupled system to have enormous energy if the original phase zero modes were non-trivially populated, which leads to an unstable time evolution. In this situation the lowest order phononic model is not anymore a good approximation to the Lieb-Liniger model (1) as the density fluctuations may no longer be small.

Nevertheless, one can refine the model considered here to reflect more accurately the corresponding physical process: energy will change continuously, since when we couple the systems by ramping down the separation barrier, there will be an additional term in the Hamiltonian, representing tunneling between the condensates. The density phase expansion of this term will additionally give rise to a term of the type $\hbar J \cos (\Delta \hat{\varphi})$ penalizing phase fluctuations $\Delta \hat{\varphi}=\hat{\varphi}_{L}-\hat{\varphi}_{R}$ ranging over the interface. The action of this term is to induce phase locking between the two condensates being merged together, see Refs. [33,34,159,167] for experimental discussions and references therein for the theoretical overview. The large coupling expansion of this term motivates the effective model we used in the numerical simulations

$$
\hat{H}\left[\rho_{0}\right]=\hat{H}_{\mathrm{P}}\left[\rho_{0}\right]+h \int d z J(z) \rho_{0}(z) \hat{\varphi}(z)^{2} .
$$

\section{a. Analytical derivation of gapping out the zero mode in the homogeneous phase-locking model}

In the experiment the phase-locking term will be acting around the interface. For the case of large extension of this coupling (or two sideways coupled systems $[33,168]$ ) it is 
instructive to consider $J=$ const throughout the condensates. In this case additionally taking $\rho_{0}=$ const we can analytically see that this term effectively gaps out the phase zero mode. One way to see this it by noticing that this term amounts to add a (small) "mass" term to Eq. (C32), leading to the modified Sturm-Liouville problem

$$
\omega_{k}^{2} \varphi_{k}(z)=-\frac{g}{m} \partial_{z}\left[\rho_{0}(z) \partial_{z} \varphi_{k}(z)\right]+2 J \rho(z) \varphi_{k}(z),
$$

which effectively removes the zero mode.

Let us show this specifically in the case when all coupling constants do not vary over the condensate of length $L$, i.e., $g(z)=g, \rho_{0}(z)=\rho_{0}$ and $J(z)=J$. The Hamiltonian then reads

$$
\hat{H}=\int_{0}^{L} d z\left\{\frac{\hbar^{2} \rho_{0}}{2 m}\left[\partial_{z} \hat{\varphi}(z)\right]^{2}+\frac{g}{2} \delta \hat{\varrho}(z)^{2}+\hbar J \rho_{0} \hat{\varphi}(z)^{2}\right\},
$$

and has no zero modes unless $J=0$. In this case the eigenfrequencies read

$$
\omega_{k}=\frac{\pi c k}{L}
$$

with the speed of sound given by $\sqrt{g \rho_{0} / m}$. To bring the Hamiltonian to the normal form, we define the squeezing constants

$$
\alpha_{k}=\sqrt{\frac{\hbar \omega_{k}}{g}}+\delta_{k, 0}
$$

from which we define for $k>0$

$$
\begin{aligned}
\delta \hat{\varrho}_{k} & =\alpha_{k} \sqrt{\frac{2}{L}} \int_{0}^{L} d z \cos (\pi k z / L) \delta \hat{\varrho}(z) \text { and } \\
\hat{\varphi}_{k} & =\alpha_{k}^{-1} \sqrt{\frac{2}{L}} \int_{0}^{L} d z \cos (\pi k z / L) \hat{\varphi}(z)
\end{aligned}
$$

and

$$
\delta \hat{\varrho}_{0}:=\sqrt{\frac{1}{2 L}} \int_{0}^{L} d z \delta \hat{\varrho}(z) \text { and } \hat{\varphi}_{0}:=\sqrt{\frac{1}{2 L}} \int_{0}^{L} d z \hat{\varphi}(z),
$$

which stand out by having different normalization constants and would be the zero-mode operators for $J=0$.
Using standard trigonometric integrals we find

$$
\begin{aligned}
\int_{0}^{L} d z \delta \hat{\varrho}(z)^{2} & =\sum_{k=0}^{\infty} \alpha_{k}^{2} \delta \hat{\varrho}_{k}^{2} \text { and } \int_{0}^{L} d z\left[\partial_{z} \hat{\varphi}(z)\right]^{2} \\
& =\sum_{k>0} \frac{\pi^{2} k^{2}}{L^{2} \alpha_{k}^{2}} \hat{\varphi}_{k}^{2} \text { and } \int_{0}^{L} d z \hat{\varphi}(z)^{2} \\
& =\sum_{k=0}^{\infty} \alpha_{k}^{-2} \hat{\varphi}_{k}^{2} .
\end{aligned}
$$

Therefore,

$$
\begin{aligned}
\hat{H}= & \sum_{k=1}^{\infty} \frac{\hbar \omega_{k}}{2}\left[\delta \hat{\varrho}_{k}^{2}+\hat{\varphi}_{k}^{2}\right]+\frac{g}{2} \delta \hat{\varrho}_{0}^{2} \\
& +\hbar J \rho_{0} \sum_{k=1}^{\infty} \alpha_{k}^{-2} \hat{\varphi}_{k}^{2}+\hbar J \rho_{0} \hat{\varphi}_{0}^{2} .
\end{aligned}
$$

Further defining

$$
\zeta_{k}:=\frac{4 g J \rho_{0}}{\hbar^{2} \omega_{k}^{2}}
$$

for $k>0$ we obtain the form

$$
\hat{H}=\sum_{k=1}^{\infty} \frac{\hbar \omega_{k}}{2}\left[\delta \hat{\varrho}_{k}^{2}+\left(1+\zeta_{k}\right) \hat{\varphi}_{k}^{2}\right]+\frac{g}{2} \delta \hat{\varrho}_{0}^{2}+\hbar J \rho_{0} \hat{\varphi}_{0}^{2} .
$$

Thus, the $k=0$ eigenmode of $\hat{H}$ has the eigenfrequency $\omega_{k=0}=\hbar \sqrt{g J \rho_{0}}$ and is not a zero mode when $J \neq 0$. We also see that there is additionally a squeezing interaction, which decays for $k \rightarrow \infty$. Figure 11 shows plots of merging for different values of $J$ when assuming that the phase-locking term acts homogeneously in space. Figure 12 shows merging obtained by artificially removing the zero mode, in order to highlight its contribution to the excitations present during the merging.

\section{b. Justification of the phase-locking model}

Finally we provide a justification for the phenomenological model above. The argument is based on the theoretical observation from Ref. [35] that a potential barrier is effectively transparent for low-frequency excitations. This hints that we can phase-lock systems in order to reduce the impact of excitations coming from zero-mode coupling and once this is done one can reduce the barrier further to increase heat transmission.

We consider a quasicondensate of mean density $\rho_{0}$ in a box of length $2 L \gg 2 a$ (below we set $L \rightarrow \infty$ for simplicity) with a barrier extending from $z=-a$ to $z=a$ and having a finite height, which exceeds the chemical 
(a)

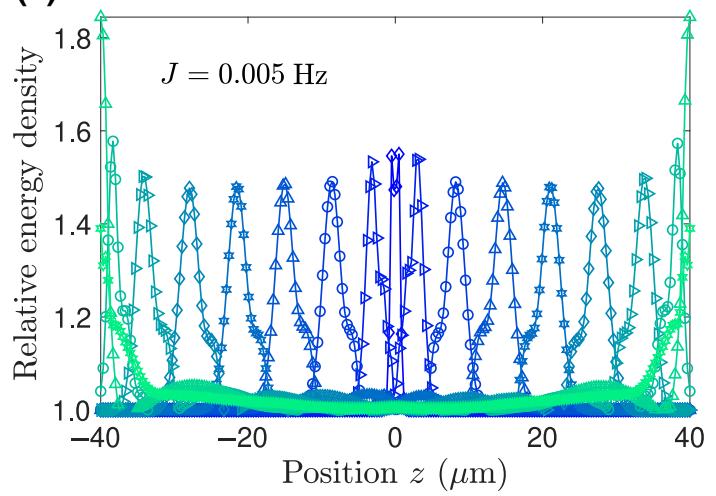

(b)

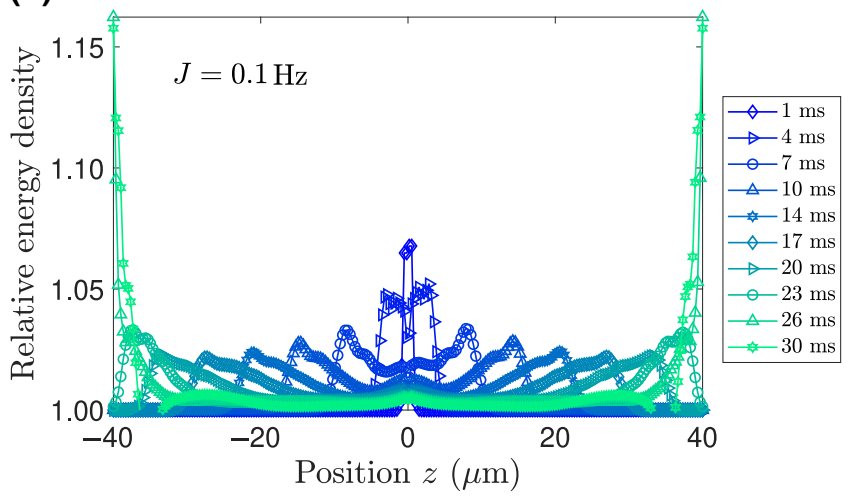

FIG. 11. Coupling of two quasicondensates for different initial phase-locking. Similarly to the main text we consider the phase locking to act with constant strength along each of the condensates and show the influence of other values of $J$ on the outcome of merging. (a) For a low value of $J=0.005 \mathrm{~Hz}$ there is substantially more excitations compared to the value $J=0.01 \mathrm{~Hz}$ used for all plots presented in the main text. (b) On the other hand, for larger values of phase locking such as $J=0.1 \mathrm{~Hz}$ the excitations become suppressed as the phase zero mode acquires a larger energetic penalty and its initial thermal second moments are smaller. Note, that when increasing the tunnel coupling $J$ further, one expects a non-Gaussian regime due to non-negligible interactions stemming from the full cosine potential [20].

potential by $U_{B}$. Moreover, we assume that tunnel coupling between zero modes of the left and right quasicondensates is negligible. This means that the background density and low-energy excitations feel a hard wall at $z=-a$ for the left quasicondensate and at $z=a$ for the right quasicondensate. For concreteness, let us focus on the left quasicondensate, and it is clear that similar considerations apply also for the right one. The background solution in the bulk (far from the leftmost end) is $\Psi_{0, L}(z)=\sqrt{\rho_{0}} \tanh [-(z+$ a) $\left./ \xi_{h}\right], z<-a$, where $\xi_{h}=\hbar /(m c)$ is the healing length. Considering the first order in matter field fluctuation $\hat{\Psi}_{L}=$ $\Psi_{0, L} \hat{\mathbb{1}}+\delta \hat{\Psi}_{L}$ around the full stationary solution the Hamiltonian term corresponding to atom scattering becomes (neglecting a constant term)

$$
\hat{V}_{L}=2 g \int_{-\infty}^{-a} d z\left|\Psi_{0, L}\right|^{2} \delta \hat{\Psi}_{L}^{\dagger}(z) \delta \hat{\Psi}_{L}(z) .
$$

This term, because of the large gradient of $\Psi_{0, L}$ in the two bulks, couples low-energy excitations to high-energy ones. The former can be represented as $\delta \hat{\Psi}_{L}(z, t) \tanh [-(z+$ a) $\left./ \xi_{h}\right]$, where $\delta \hat{\Psi}_{L}(z, t)$ is subject to Neumann boundary conditions at $z=-a$. The factor $\tanh \left[-(z+a) / \xi_{h}\right]$ follows from considering the adiabatic solution of the time-dependent GPE for excitations with a frequency much lower than $g \rho_{0} / \hbar$ and makes the fluctuation vanishing at the wall. Let us now consider the propagation
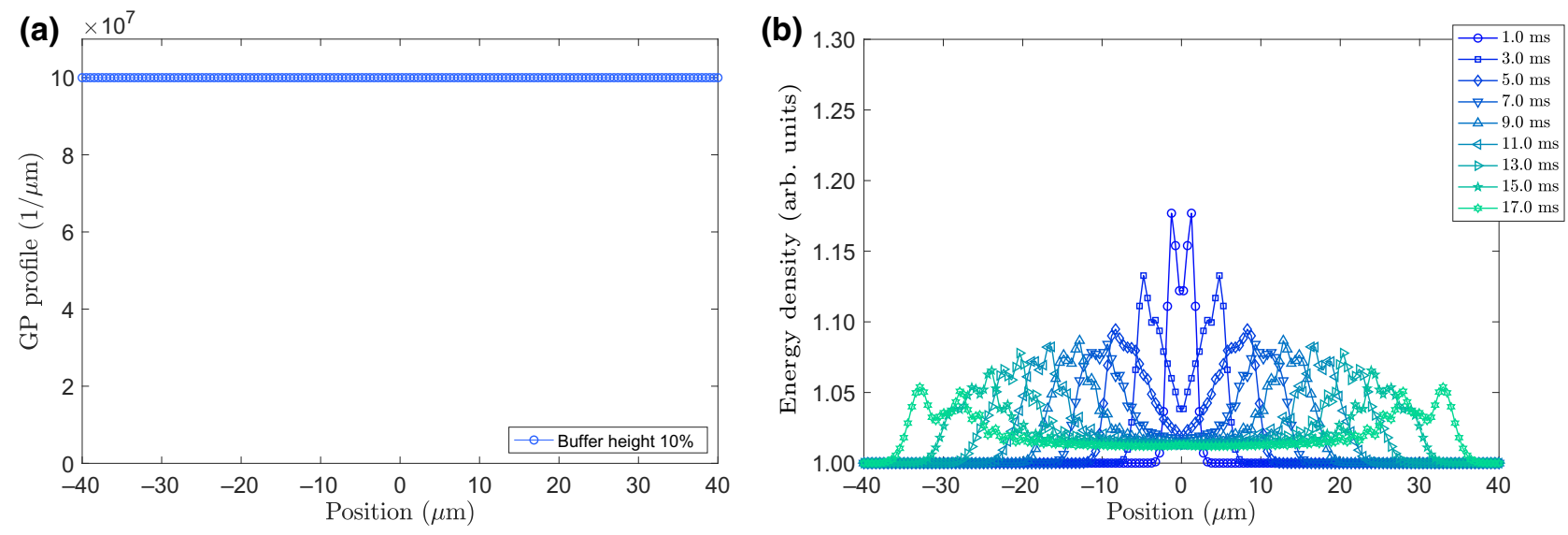

FIG. 12. Merging of homogeneous systems where the zero mode is artificially removed from the evolution. Using the eigenmode decomposition for $J=0$ we set $\delta \hat{\varrho}_{0}=\hat{\varphi}_{0}=0$ in the Hamiltonian that governs the merging and compute the initial state using a pseudoinverse disregarding the zero mode. We see a behavior, both qualitative and quantitative, similar to merging with a regularization coupling chosen as $J=0.01 \mathrm{~Hz}$. 
of high-energy excitations. The high-energy, particlelike excitations propagating from the left $(>)$ or from the right $(<)$ are parametrized with the following set of orthogonal functions:

$$
\begin{aligned}
& \psi_{k}^{>}(z) \sim\left\{\begin{array}{ll}
e^{i k z}+i \sin \beta_{k} e^{i \alpha_{k}} e^{-i k z}, & z<-a \\
\cos \beta_{k} e^{i \alpha_{k}} e^{i k z}, & z>a
\end{array},\right. \\
& \psi_{k}^{<}(z) \sim\left\{\begin{array}{ll}
\cos \beta_{k} e^{i \alpha_{k}} e^{-i k z}, & z<-a \\
e^{-i k z}+i \sin \beta_{k} e^{i \alpha_{k}} e^{i k z}, & z>a
\end{array} .\right.
\end{aligned}
$$

Here, $\alpha_{k}, \beta_{k}$ parametrize the transmission and reflection amplitudes $(k>0)$ and we also have $\left\langle\psi_{k}^{5} \mid \psi_{k^{\prime}}^{\varsigma^{\prime}}\right\rangle=$ $\delta_{\varsigma^{\prime}, \varsigma} \delta\left(k^{\prime}-k\right), \varsigma^{\prime}, \varsigma=>,<$.

We can expect that $\cos \beta_{k}$ rapidly increases from almost 0 to almost 1 , when $k$ approaches $q_{B}=\sqrt{2 m U_{B}} / \hbar$. We apply a perturbative approach, with the Hamiltonian with hard walls at $z= \pm a$ being the unperturbed Hamiltonian and the Hamiltonian with the barrier of a finite height being the perturbed one. The second-order approximation yields the following term coupling low-energy excitation fields in the left and right quasicondensates:

$$
\hat{H}_{L R}=-\int_{-\infty}^{-a} d z \int_{a}^{\infty} d z^{\prime} \mathcal{J}\left(z, z^{\prime}\right)\left[\delta \hat{\Psi}_{L}^{\dagger}(z) \delta \hat{\Psi}_{R}\left(z^{\prime}\right)+\text { H.c. }\right],
$$

where the effective coupling coefficient is

$$
\begin{aligned}
\mathcal{J}\left(z, z^{\prime}\right)= & \frac{4\left(g \rho_{0}\right)^{2}}{U_{B}} \tanh ^{3}\left(-\frac{z+a}{\xi_{h}}\right) \tanh ^{3}\left(\frac{z^{\prime}-a}{\xi_{h}}\right) \\
& \times \int_{q_{B}}^{\infty} \frac{d k}{\pi} \frac{q_{B}^{2}}{k^{2}}\left\{\cos \left[k\left(z+z^{\prime}\right)\right]\right. \\
& \left.+\cos \left[k\left(z-z^{\prime}\right)-\alpha_{k}-\beta_{k}\right]\right\} .
\end{aligned}
$$

In the harmonic approximation, we replace $\delta \hat{\Psi}_{R}^{\dagger} \delta \hat{\Psi}_{L} \approx$ $(1 / 2) \rho_{0}\left(\hat{\varphi}_{L}-\hat{\varphi}_{R}\right)^{2}$ (after neglecting density fluctuations [22]) obtaining

$$
\hat{H}_{L R} \approx \mathrm{const}+\frac{1}{2} \int_{-\infty}^{-a} d z \int_{a}^{\infty} d z^{\prime} \mathcal{J}\left(z, z^{\prime}\right) \rho_{0}\left[\hat{\varphi}(z)-\hat{\varphi}\left(z^{\prime}\right)\right]^{2}
$$

$$
\begin{aligned}
\approx & \frac{1}{2} \int_{-\infty}^{-a} d z \tilde{\mathcal{J}}(z) \rho_{0} \hat{\varphi}(z)^{2}+\frac{1}{2} \int_{a}^{\infty} d z \tilde{\mathcal{J}}(z) \rho_{0} \hat{\varphi}(z)^{2} \\
& -\int_{-\infty}^{-a} d z \int_{a}^{\infty} d z^{\prime} \mathcal{J}\left(z, z^{\prime}\right) \rho_{0} \hat{\varphi}(z) \hat{\varphi}\left(z^{\prime}\right),
\end{aligned}
$$

which motivates the phenomenological model (C38). Note that in Eq. (C38) we further neglect the last interaction term $-\int_{-\infty}^{-a} d z \int_{a}^{\infty} d z^{\prime} \mathcal{J}\left(z, z^{\prime}\right) \rho_{0} \hat{\varphi}(z) \hat{\varphi}\left(z^{\prime}\right)$.

\section{c. Phase diffusion after removing the phase-locking interaction}

We consider a thermal state with $J \neq 0$ with full support and finite energy penalty on the $k=0$ eigenmode (for $J=0$ it is the phase zero mode). We thus have $\left\langle\hat{\varphi}_{0}^{2}\right\rangle \propto k_{B} T$ and $\left\langle\delta \hat{\varrho}_{0}^{2}\right\rangle \propto k_{B} T$ similar to the ordinary $k>0$ modes. We then perform a quench to $J=0$, which means $\hat{\varphi}_{0} \rightarrow \hat{\varphi}_{\mathrm{ZM}}$ and $\delta \hat{\varrho}_{0} \rightarrow \delta \hat{\varrho}_{\mathrm{ZM}}$, and observe how the phase zero mode grows given by the equation

$$
\left\langle\hat{\varphi}_{\mathrm{ZM}}^{2}(t)\right\rangle=\left\langle\hat{\varphi}_{0}^{2}\right\rangle+\frac{g^{2} t^{2}}{\hbar^{2}}\left\langle\delta \hat{\varrho}_{0}^{2}\right\rangle .
$$

Figure 13 demonstrates the effect of taking into account zero-mode phase diffusion during the merging process.

\section{Sudden merging in the continuous QFT limit and additional checks of the numerical simulation}

Let us also briefly discuss here how our simulations compare with the continuum limit $\Delta z \rightarrow 0$. Essentially, besides the fact that the field operators themselves have the appropriate continuum limit, in the static case we are also interested in recovering the spectrum and the eigenfunctions of the Hamiltonian (5) to some extent. In particular, let us consider two types of density profiles $\rho_{0}(z)$ that are piecewise constant functions: (1) two disconnected parts of lengths $L_{A}$ and $L_{B}$ on intervals $\left[-L_{A}, 0\right)$ and $\left(0, L_{B}\right]$, where the interface is at $z=0$, which corresponds to the Hamiltonian $\hat{H}_{A \mid B}$ and (2) a single system with length $L_{A B}=$ $L_{A}+L_{B}$ where the high wall at the interface has been removed, which corresponds to the Hamiltonian $\hat{H}_{A B}$. We further impose Neumann (open) boundary conditions at all boundary points, i.e., $\left.\partial_{z} \varphi_{k}\right|_{-L_{A}}=\left.\partial_{z} \delta \varrho_{k}\right|_{-L_{A}}=\left.\partial_{z} \varphi_{k}\right|_{L_{B}}=$ $\left.\partial_{z} \delta \varrho_{k}\right|_{L_{B}}=0$, and similarly for the point $z=0$ in case (1). In such cases, solutions to Eq. (C32) can be easily found on each interval and are given by usual oscillatory functions with a linear dispersion relation

$$
\omega_{k}(L)=\pi c k / L
$$

where $c=\sqrt{\rho_{0} g / m}$ is the speed of sound, $k$ is an integer number and it also depends on the length of the corresponding interval $L \in\left\{L_{A}, L_{B}, L_{A B}\right\}$. In case (1) we have the two solutions for $k>0$ 

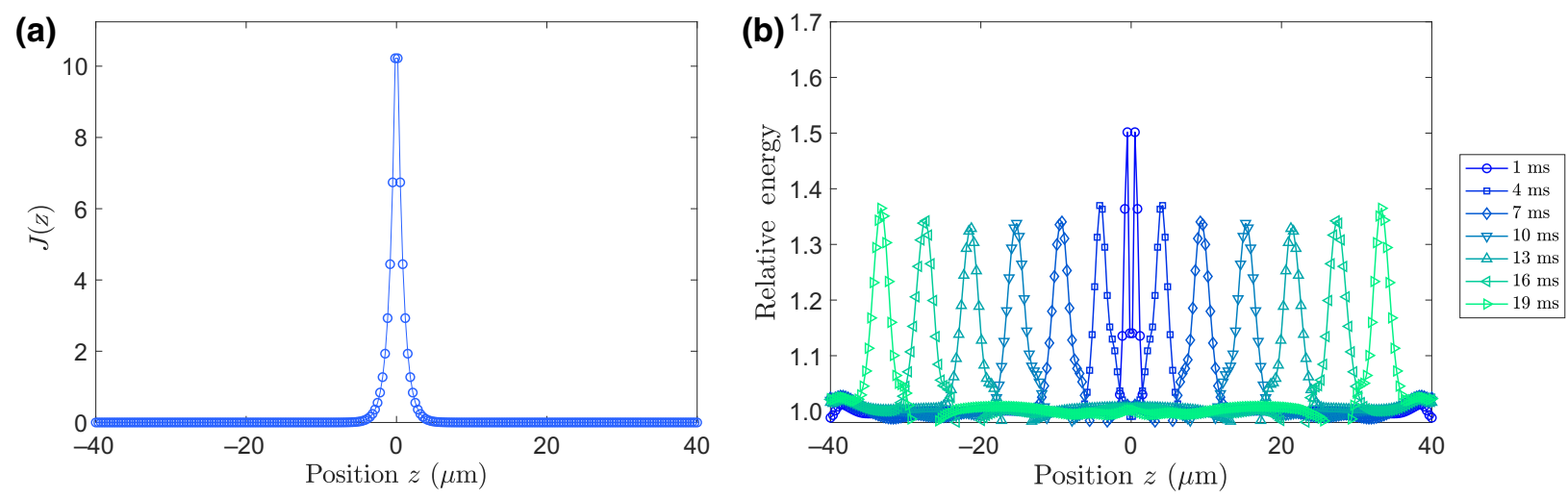

FIG. 13. The contribution of zero-mode phase diffusion to energy excitations during merging. Two systems are prepared in thermal, phase-locked states according to the localized coupling $J$ shown in (a) with overall strength similar to the value used in the main text $\bar{J}=\frac{1}{L} \int d z J(z) \approx 0.25 \mathrm{~Hz}$. The magnitude of $J$ at the interface can be tuned in experiments by the barrier parameters. After the preparation phase, $J$ is then quenched to 0, signifying a decoupling of the two systems into independent, gapless Luttinger liquids, where the zero phase mode has no contribution to energy and diffuses according to Eq. (C55) for a total time of $25 \mathrm{~ms}$. When the systems are again merged, as shown in (b), we see that large excitations can potentially be induced due to the diffusion of the zero mode. In order to minimize the energy of excitations one should choose a large $J$ in the beginning (meaning a stronger phase locking during preparation), and design the cycle times to be shorter. The amount of excitations here is an overestimate as we did not include in the modeling the possibility of phase locking the condensates before merging, this process could involve strong correlations via the Josephson junction and could counteract phase diffusion.

$$
\begin{gathered}
\delta \varrho_{2 k-1}^{A \mid B}(z)=\varphi_{2 k-1}^{A \mid B}(z)=\left\{\begin{array}{cc}
\sqrt{\frac{2}{L_{A}}} \cos \left[\pi k\left(z+L_{A}\right) / L_{A}\right] & \text { for } z \in\left[-L_{A}, 0\right], \\
0 & \text { for } z \in\left(0, L_{B}\right]
\end{array}\right. \\
\delta \varrho_{2 k}^{A \mid B}(z)=\varphi_{2 k}^{A \mid B}(z)=\left\{\begin{array}{cc}
0 & \text { for } z \in\left[-L_{A}, 0\right] \\
\sqrt{\frac{2}{L_{B}}} \cos \left[\pi k\left(z-L_{B}\right) / L_{B}\right] & \text { for } z \in\left(0, L_{B}\right],
\end{array}\right.
\end{gathered}
$$

with corresponding dispersion relations, respectively, $\omega_{2 k-1}=\pi c k / L_{A}$ and $\omega_{2 k}=\pi c k / L_{B}$. Note that in the case $L_{A}=L_{B}$ there is a degeneracy between even and odd modes.

For case (2), instead, we have the solutions

$$
\delta \varrho_{k}^{A B}(z)=\varphi_{k}^{A B}(z)=\sqrt{\frac{2}{L_{A B}}} \cos \left[\pi k\left(z+L_{A}\right) / L_{A B}\right],
$$

with dispersion relation $\omega_{k}^{A B}=\pi c k / L_{A B}$. The agreement of the dispersion relation and the profile of the eigenmode functions in the static case can be observed in Figs. 14 and 15 where a comparison with the discretized homogeneous model with or without a trapezelike buffer region is shown.

Afterwards, let us try to compare the dynamics of the merging QTP with its continuous quantum field limit. First of all, we observe that the initial state in the continuous QFT, i.e., the thermal state of the QFT limit of the split Hamiltonian, would have the spectrum of the covariance matrix given by $\left\langle\left(\hat{\varphi}_{k}\right)^{2}+\left(\delta \hat{\varrho}_{k}\right)^{2}\right\rangle=2\left\langle\hat{n}_{k}\right\rangle+1$, where $\left\langle\hat{n}_{k}\right\rangle=1 /\left[\exp \left(\beta \omega_{k}\right)-1\right]$ are the normal-mode occupation numbers, given by the usual Bose-Einstein distribution. Then, from the fact that for our initial state we have $\left\langle\hat{a}_{k}^{\dagger} \hat{a}_{l}^{\dagger}+\hat{a}_{k} \hat{a}_{l}\right\rangle=0$ and $\left\langle\hat{a}_{k}^{\dagger} \hat{a}_{l}+\hat{a}_{k} \hat{a}_{l}^{\dagger}\right\rangle=\left(2\left\langle\hat{n}_{k}\right\rangle+1\right) \delta_{k, l}$, we obtain for the initial real-space correlation matrix

$$
\begin{aligned}
C_{A-B}\left(z, z^{\prime}, t=0\right)= & C^{\rho \rho}\left(z, z^{\prime}\right) \oplus C^{\phi \phi}\left(z, z^{\prime}\right) \\
= & \sum_{k>0} \frac{\hbar \omega_{k}}{g} \delta \varrho_{k}(z) \delta \varrho_{k}\left(z^{\prime}\right)\left(2\left\langle\hat{n}_{k}\right\rangle+1\right) \\
& \oplus \sum_{k>0} \frac{g}{\hbar \omega_{k}} \varphi_{k}(z) \varphi_{k}\left(z^{\prime}\right)\left(2\left\langle\hat{n}_{k}\right\rangle+1\right),
\end{aligned}
$$

where here and in the following discussion we discard the zero mode, since in the simulations we have regularized it as discussed in Appendix C3. 

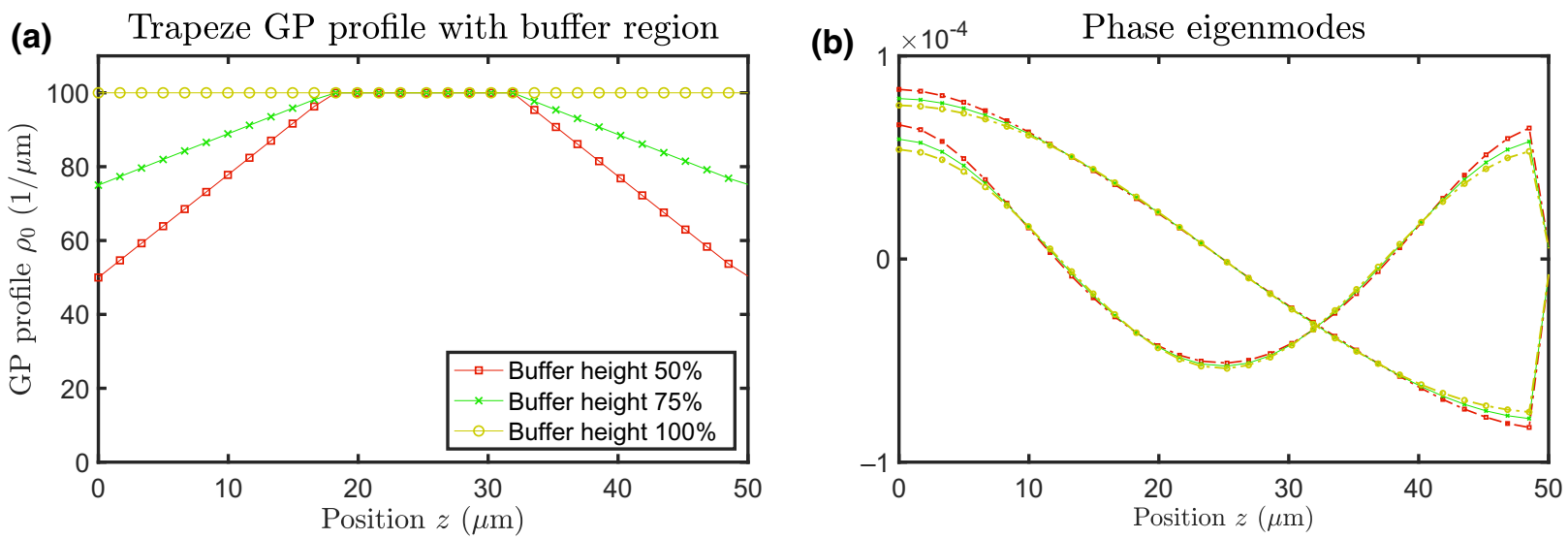

FIG. 14. Influence of the buffer region on eigenmodes. (a) Using the lattice discretization various inhomogenous GP profiles can be considered. (b) Phase eigenmodes for $k=1$ and $k=2$ of disjoint Hamiltonian $H_{A \mid B}$. Only the half system with nonzero eigenmode functions is shown as they vanish outside of the support of the GP profile. Qualitatively, all modes retain their oscillatory nature, though at the edges, where the inhomogeneity is the largest, there is a systematic change in the wave functions.

Clearly, the result in Eq. (C60) is very similar to the discretized case, but contains small differences in the normalmode frequencies and in the functional form of the normal modes with respect to the real-space modes. For the energy density we thus obtain

$$
\begin{aligned}
\frac{d E(z, 0)}{d z}= & \left.\frac{\hbar^{2} \rho_{0}(z)}{2 m} \partial_{z_{1}} \partial_{z_{2}} C_{A-B}^{\phi \phi}\left(z_{1}, z_{2}, t=0\right)\right|_{z_{1}=z_{2}=z} \\
& +\frac{g}{2} C_{A-B}^{\rho \rho}(z, z, t=0) \quad(\mathrm{C} 61) \\
= & \hbar \sum_{k>0}\left(\frac{\rho_{0}}{2 m} \frac{g}{\omega_{k}}\left[\partial_{z} \varphi_{k}(z)\right]^{2}+\frac{g}{2} \frac{\omega_{k}}{g} \delta \varrho_{k}^{2}(z)\right) \\
& \times\left(2\left\langle\hat{n}_{k}\right\rangle+1\right) \\
= & \begin{cases}\frac{\hbar}{L_{A}} \sum_{k \text { odd }} \omega_{k}\left(\left\langle\hat{n}_{k}\right\rangle+1 / 2\right) & \text { for } z \in\left[-L_{A}, 0\right) \\
\frac{\hbar}{L_{B}} \sum_{k \text { even }} \omega_{k}\left(\left\langle\hat{n}_{k}\right\rangle+1 / 2\right) & \text { for } z \in\left(0, L_{B}\right],\end{cases}
\end{aligned}
$$

where in the first equality we have used $\left\langle\hat{a}_{k}^{\dagger} \hat{a}_{l}+\hat{a}_{k} \hat{a}_{l}^{\dagger}\right\rangle=$ $\left(2\left\langle\hat{n}_{k}\right\rangle+1\right) \delta_{k, l}$ and in the second equality we used that

$$
\begin{aligned}
& \frac{\rho_{0}}{2 m} \frac{g}{\omega_{k}}\left[\partial_{z} \varphi_{k}(z)\right]^{2}+\frac{g}{2} \frac{\omega_{k}}{g} \delta \varrho_{k}^{2}(z)=\frac{1}{L_{A}} \frac{\omega_{k}}{2} \quad \text { for } k \text { odd }, \\
& \frac{\rho_{0}}{2 m} \frac{g}{\omega_{k}}\left[\partial_{z} \varphi_{k}(z)\right]^{2}+\frac{g}{2} \frac{\omega_{k}}{g} \delta \varrho_{k}^{2}(z)=\frac{1}{L_{B}} \frac{\omega_{k}}{2} \quad \text { for } k \text { even }
\end{aligned}
$$

for all $z$, respectively, in $\left[-L_{A}, 0\right)$ and $\left(0, L_{B}\right]$, and we have that the functions are zero otherwise. Let us now consider the time-dependent interaction. The energy density at time $t>0$ during this evolution is calculated as

$$
\begin{aligned}
\frac{d E(z, t)}{d z}= & \left.\frac{\hbar^{2} \rho_{0}(z)}{2 m} \partial_{z_{1}} \partial_{z_{2}} C_{A-B}^{\phi \phi}\left(z_{1}, z_{2}, t\right)\right|_{z_{1}=z_{2}=z} \\
& +\frac{g}{2} C_{A-B}^{\rho \rho}(z, z, t),
\end{aligned}
$$

where now we need the diagonal blocks of the correlation matrix at time $t$, namely $C_{A-B}^{\phi \phi}\left(z, z^{\prime}, t\right)=\left\langle\hat{\varphi}(z, t) \hat{\varphi}\left(z^{\prime}, t\right)\right\rangle$ and $C_{A-B}^{\rho \rho}\left(z, z^{\prime}, t\right)=\left\langle\delta \hat{\varrho}(z, t) \delta \hat{\varrho}\left(z^{\prime}, t\right)\right\rangle$, which, in turn, can be calculated from the instantaneous eigenmode functions at time $t$, that are given essentially by solving Eq. (C32), but now with different boundary conditions at the interface point $z=0$. See Figs. 8 and 9 for a plot of the lowest-lying eigenmode functions in the discretized model.

Specifically, given the eigenmode functions $\varphi_{k}^{(t)}(z)$ and $\delta \varrho_{k}^{(t)}(z)$ of the Hamiltonian at time $t$, together with the corresponding eigenmode frequencies $\omega_{k}^{(t)}$, we can find the time-evolved field operators at time $t$ as

$$
\hat{\varphi}(z, t)=-i \sum_{k} \sqrt{\frac{g}{\hbar \omega_{k}^{(t)}}} \varphi_{k}^{(t)}(z)\left(e^{i \omega_{k}^{(t)}} \hat{t}_{k}^{\dagger}-e^{-i \omega_{k}^{(t)}} \hat{t}_{k}\right),
$$

where $\hat{t}_{k}$ and $\hat{t}_{k}^{\dagger}$ are the instantaneous creation and annihilation, obtained with a (real) Bogoliubov transformation

$$
\hat{t}_{k}=\sum_{l} u_{k, l} \hat{a}_{l}+v_{k, l} \hat{a}_{l}^{\dagger},
$$

from those at $t=0$. The Bogoliubov coefficients are obtained by imposing that the operators $\hat{\varphi}(z, t=0)$ and 


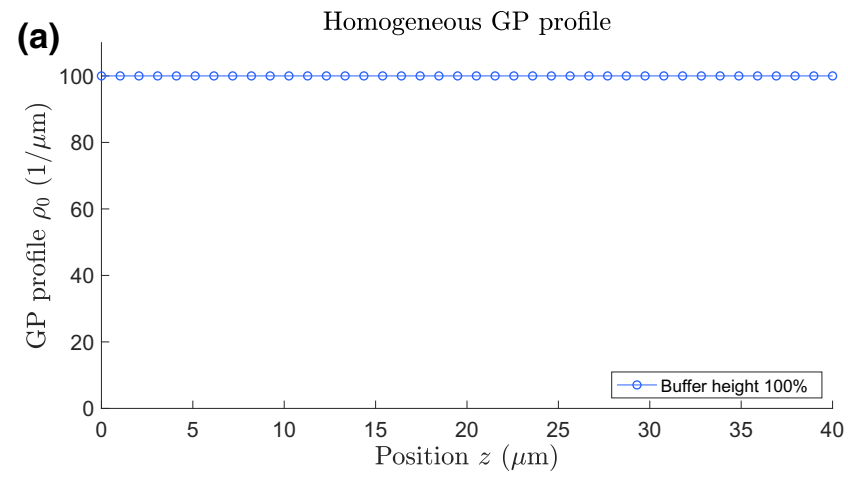

(b)

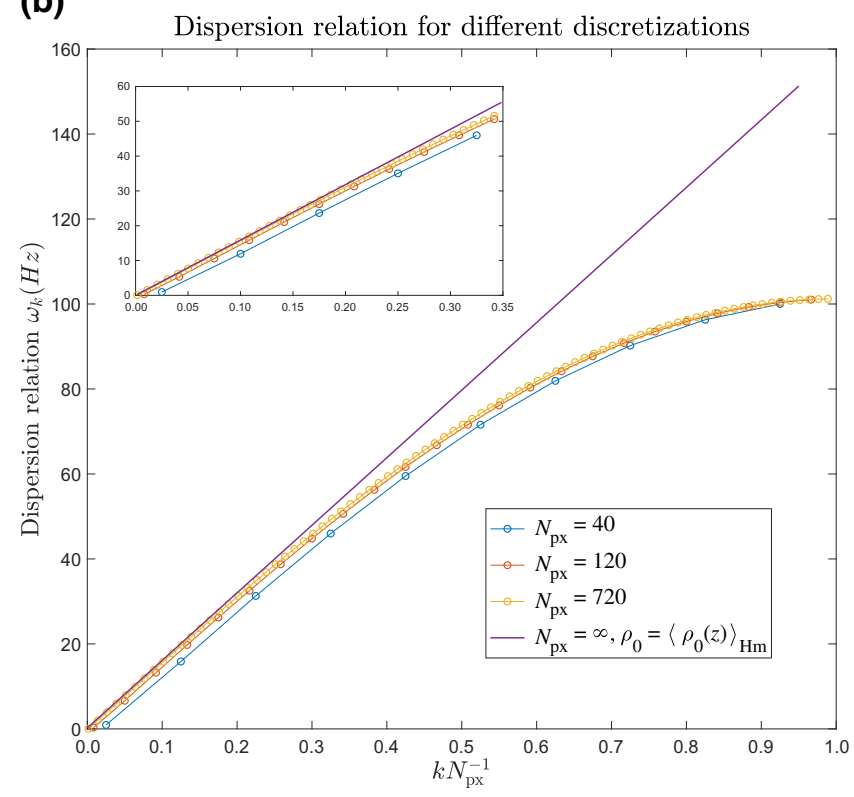

FIG. 15. Dispersion relations for the homogeneous GP profile. For a homogeneous profile (top) we find that for high momentum modes the dispersion relation is no longer approximately linear due to the lattice discretization. This leads to the dispersion of the wave packets during for example merging. The inset shows that approximately $30 \%$ of the low-energy modes already gives rise to a good approximation to the continuum limit, especially for discretizations above the order of approximately 100 pixels, which is the resolution at where our simulations were performed (see also Fig. 16).

$\delta \hat{\varrho}(z, t=0)$ coincide with the initial ones, i.e.,

$$
\begin{aligned}
& -i \sum_{k} \sqrt{\frac{g}{\hbar \omega_{k}^{(t)}}} \varphi_{k}^{(t)}(z) \sum_{l}\left(u_{k, l}-v_{k, l}\right)\left(\hat{a}_{l}^{\dagger}-\hat{a}_{l}\right) \\
& =-i \sum_{k} \sqrt{\frac{g}{\hbar \omega_{k}^{(0)}}} \varphi_{k}^{(0)}(z)\left(\hat{a}_{k}^{\dagger}-\hat{a}_{k}\right),
\end{aligned}
$$

and can be extracted from the scalar products between the initial and the instantaneous eigenmode functions:

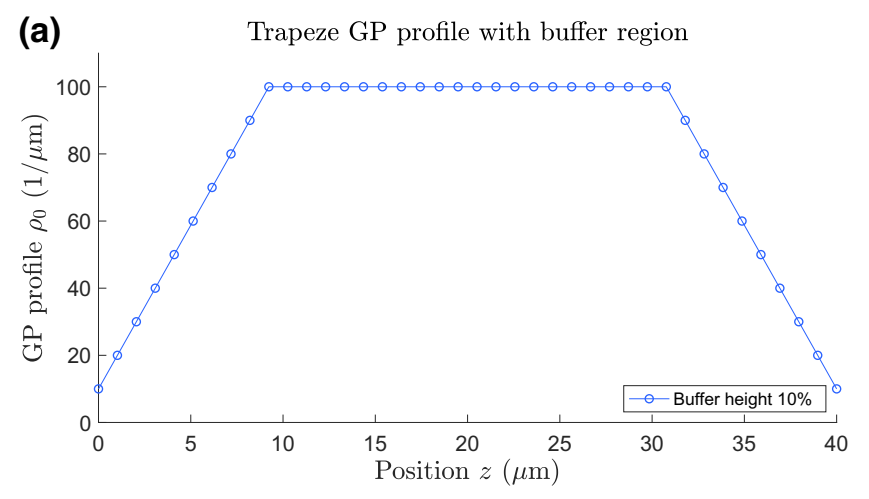

(b)

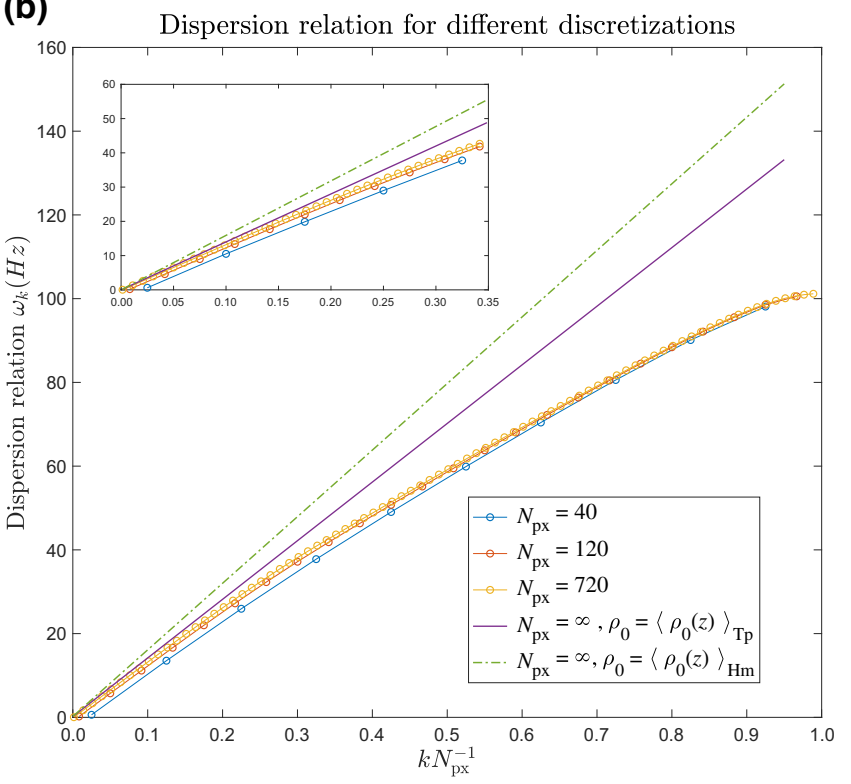

FIG. 16. Dispersion relations for the trapeze GP profile. When compared to Fig. 15, the dispersion relation for higher modes is closer to a linear curve, especially for high-momentum modes. This is why when using such a trapeze GP model when putting condensates in heat contact, we observe wave packets undergoing significantly less dispersion. Since less atoms are considered in this condensate due to the trapeze-shaped profile (TP), the low-lying energy modes are better approximated by the continuum limit assuming a homogeneous condensate with GP profile at $\rho_{0}=\left\langle\rho_{0}(z)\right\rangle_{\mathrm{Tp}}=$ const.

$$
\begin{aligned}
u_{k, l}-v_{k, l} & =\sqrt{\frac{\omega_{k}^{(t)}}{\omega_{l}^{(0)}}}\left\langle\varphi_{l}^{(0)}(z), \varphi_{k}^{(t)}(z)\right\rangle \\
u_{k, l}+v_{k, l} & =\sqrt{\frac{\omega_{l}^{(0)}}{\omega_{k}^{(t)}}}\left\langle\delta \varrho_{l}^{(0)}(z), \delta \varrho_{k}^{(t)}(z)\right\rangle,
\end{aligned}
$$

where the relation on the right comes from a similar condition on the $\delta \varrho_{k}$ eigenfunctions. Thus, substituting all of the above relations, the evolved correlation matrices can be obtained through the formulas 

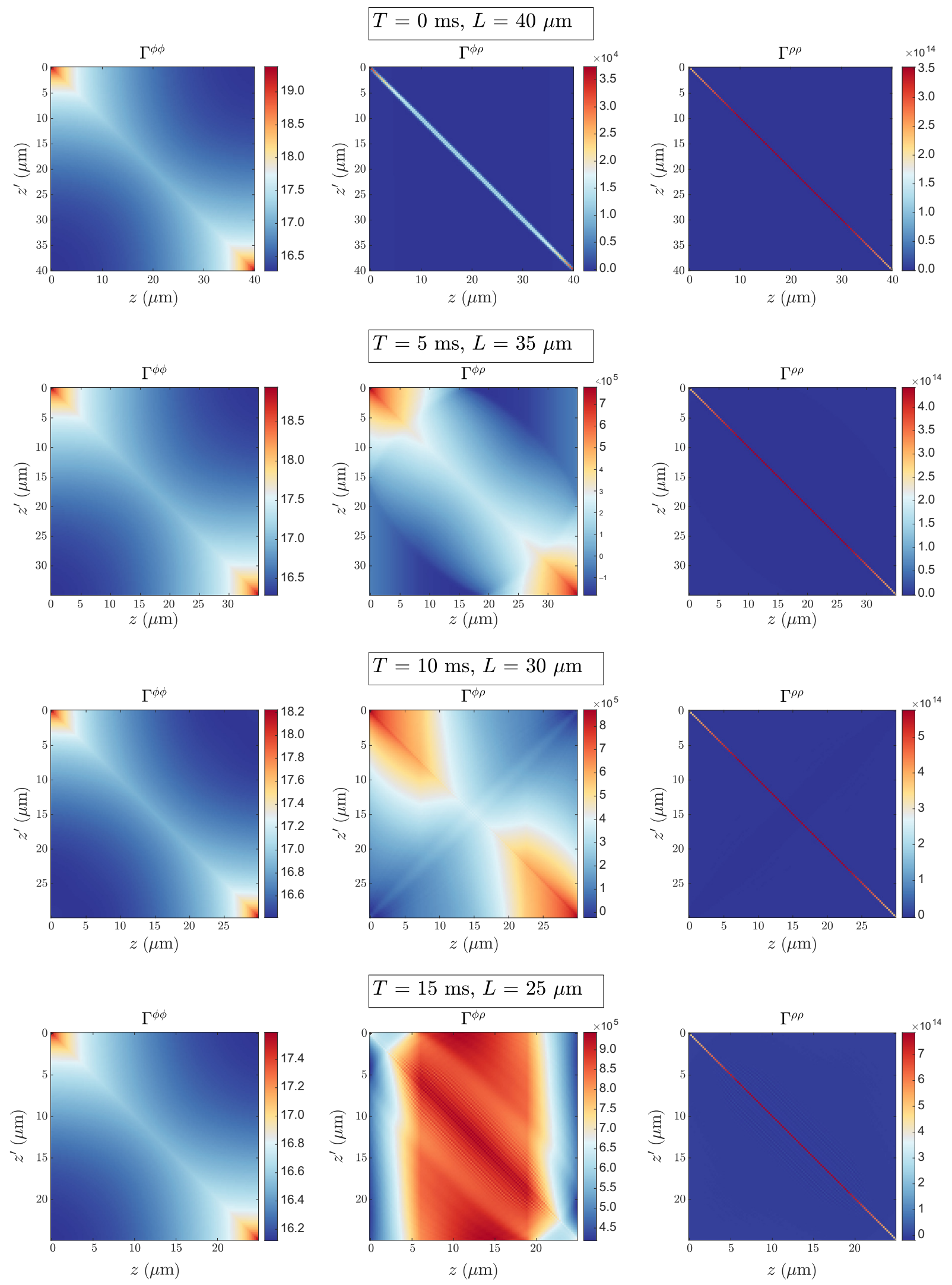

FIG. 17. Covariance matrix of the piston while being compressed over a period of $20 \mathrm{~ms}$ from $L(0)=40 \mu \mathrm{m}$ to $L\left(t_{\text {comp }}\right)=20 \mu \mathrm{m}$. The squeezing of eigenmodes can also be seen in real space as shown here: one finds that the overall magnitude of phase fluctuations decreases while for the density fluctuations it increases. The cross-correlations do not contribute to the energy of the piston but their presence signifies that the system is not thermal during compression. 


$$
\begin{aligned}
C_{A-B}^{\phi \phi}\left(z, z^{\prime}, t\right)= & \sum_{k, l, r}\left(2\left\langle\hat{n}_{k}\right\rangle+1\right) \frac{g}{\hbar \sqrt{\omega_{l}^{(t)} \omega_{r}^{(t)}}} \varphi_{l}^{(t)}(z) \varphi_{r}^{(t)}\left(z^{\prime}\right) 2\left\{\left(u_{l, k} u_{r, k}+v_{l, k} v_{r, k}\right) \cos \left[\left(\omega_{r}^{(t)}-\omega_{l}^{(t)}\right) t\right]\right. \\
& \left.-\left(u_{l, k} v_{r, k}+v_{l, k} u_{r, k}\right) \cos \left[\left(\omega_{r}^{(t)}+\omega_{l}^{(t)}\right) t\right]\right\},
\end{aligned}
$$

and

$$
\begin{aligned}
C_{A-B}^{\rho \rho}\left(z, z^{\prime}, t\right)= & \sum_{k, l, r}\left(2\left\langle\hat{n}_{k}\right\rangle+1\right) \frac{\hbar \sqrt{\omega_{l}^{(t)} \omega_{r}^{(t)}}}{g} \delta \varrho_{l}^{(t)}(z) \delta \varrho_{r}^{(t)}\left(z^{\prime}\right) 2\left\{\left(u_{l, k} u_{r, k}+v_{l, k} v_{r, k}\right) \cos \left[\left(\omega_{r}^{(t)}-\omega_{l}^{(t)}\right) t\right]\right. \\
& \left.+\left(u_{l, k} v_{r, k}+v_{l, k} u_{r, k}\right) \cos \left[\left(\omega_{r}^{(t)}+\omega_{l}^{(t)}\right) t\right]\right\}
\end{aligned}
$$

and finally we obtain the expression for the energy density by plugging all of this into Eq. (C65)

$$
\begin{aligned}
\frac{d E(z, t)}{d z}= & \hbar \sum_{k, l, r}\left(\left\langle\hat{n}_{k}\right\rangle+\frac{1}{2}\right)\left(S_{l, r}^{(t)}(z)\left\{\left(u_{l, k} u_{r, k}+v_{l, k} v_{r, k}\right) \cos \left[\left(\omega_{r}^{(t)}-\omega_{l}^{(t)}\right) t\right]\right\}\right. \\
& \left.+D_{l, r}^{(t)}(z)\left\{\left(u_{l, k} v_{r, k}+v_{l, k} u_{r, k}\right) \cos \left[\left(\omega_{r}^{(t)}+\omega_{l}^{(t)}\right) t\right]\right\}\right),
\end{aligned}
$$

where, to shorten the notation, we define the quantities

$$
\begin{aligned}
& S_{l, r}^{(t)}(z):=\left\{\frac{\rho_{0}(z) g}{m \sqrt{\omega_{l}^{(t)} \omega_{r}^{(t)}}}\left[\partial_{z} \varphi_{l}^{(t)}(z)\right]\left[\partial_{z} \varphi_{r}^{(t)}(z)\right]+\sqrt{\omega_{l}^{(t)} \omega_{r}^{(t)}} \delta \varrho_{l}^{(t)}(z) \delta \varrho_{r}^{(t)}(z)\right\}, \\
& D_{l, r}^{(t)}(z):=\left\{\sqrt{\omega_{l}^{(t)} \omega_{r}^{(t)}} \delta \varrho_{l}^{(t)}(z) \delta \varrho_{r}^{(t)}(z)-\frac{\rho_{0}(z) g}{m \sqrt{\omega_{l}^{(t)} \omega_{r}^{(t)}}}\left[\partial_{z} \varphi_{l}^{(t)}(z)\right]\left[\partial_{z} \varphi_{r}^{(t)}(z)\right]\right\},
\end{aligned}
$$

which depend only on the instantaneous eigenfunctions and eigenfrequencies. Hence, to calculate the energy density at time $t$ we just need the additional calculation of the Bogoliubov coefficients.

As an illustrative example, let us now consider the case $L_{A}=L_{B}=L$ and in which we quench directly to the full $\hat{H}_{A B}$ at $t=0$. In such a case we have that the eigenmode functions at $t=0^{+}$are given by Eq. (C59) with $L_{A B}=2 L$ and the corresponding eigenfrequencies are $\omega_{k}^{(+)}=\pi c k / 2 L$ with the same sound velocity $c$, which are just half of the corresponding odd frequencies at $t=0^{-}$. Note, however, that at $t=0^{-}$there is a degeneracy, such that the $\omega_{k}$ with odd $k$ have the same values as the even $k$. Thus, $\omega_{k}^{(+)}$coincide with the even eigenfrequencies at $t=0^{-}$. The quantities Eq. (C73) at time $t=0^{+}$read

$$
\begin{aligned}
& S_{l, r}^{(+)}(z)=\frac{\pi c}{2 L} \sqrt{l r} \sin [\pi(l+r)(z+L) / 2 L], \\
& D_{l, r}^{(+)}(z)=\frac{\pi c}{2 L} \sqrt{l r} \cos [\pi(l+r)(z+L) / 2 L] .
\end{aligned}
$$

Furthermore, the Bogoliubov coefficients satisfy

$$
\begin{aligned}
& u_{2 k-1, l}=\frac{1}{2} \frac{\omega_{l}^{(+)}+\omega_{k}^{(0)}}{\sqrt{\omega_{k}^{(0)} \omega_{l}^{(+)}}}, \quad O_{k, l}=\frac{2 k+l}{\sqrt{2 k l}}, \quad O_{k, l}=u_{2 k, l}, \\
& v_{2 k-1, l}=\frac{1}{2} \frac{\omega_{l}^{(+)}-\omega_{k}^{(0)}}{\sqrt{\omega_{k}^{(0)} \omega_{l}^{(+)}}}, \quad O_{k, l}=\frac{2 k-l}{\sqrt{2 k l}}, \quad O_{k, l}=v_{2 k, l},
\end{aligned}
$$


where

$$
O_{k, l}=\frac{\sqrt{2}}{L} \int_{0}^{L} d z \cos (\pi k z / L) \cos (\pi l z / 2 L)=\frac{\sqrt{2}}{\pi(k+l / 2)}\left(\frac{k \sin [(k-l / 2) \pi]}{k-l / 2}+(-1)^{k} \sin (l \pi / 2)\right)
$$

are the scalar products between the eigenfunctions at $t=0^{-}$and $t=0^{+}$. Plugging all of this into Eq. (C72) we finally obtain the energy density at time $t>0$ as

$$
\begin{aligned}
\frac{d E(z, t)}{d z}= & \hbar \sum_{k \text { even }} \sum_{r, l}\left(\left\langle\hat{n}_{k}\right\rangle+\frac{1}{2}\right) \frac{\pi c}{2 k L}\left[\left(4 k^{2}+l r\right) \sin [\pi(l+r)(z+L) / 2 L] \cos [\pi c t(r-l) / 2 L]\right. \\
& \left.+\left(4 k^{2}-l r\right) \cos [\pi(l+r)(z+L) / 2 L] \cos [\pi c t(r+l) / 2 L]\right]
\end{aligned}
$$

and we can see that this expression reflects a superposition of waves traveling at speed of sound $c$, and, in particular, there is no dispersion.

\section{Details of compression and expansion}

Here we give a more detailed discussion of the approximations that were involved in formulating the piston model in the main text. We consider the Lieb-Liniger model for the gas trapped in a box of changing size from $L(0)$ to $L(t)$. After the standard phononic expansion $\hat{\Psi}=\sqrt{\hat{\rho}} e^{i \hat{\theta}}$ in the long wavelength limit the Hamiltonian can be approximated as

$$
\hat{H}_{\mathrm{LL}} \approx \int_{0}^{L} d z\left[\frac{\hbar^{2}}{2 m}\left(\partial_{z} \hat{\theta}\right) \hat{\rho}\left(\partial_{z} \hat{\theta}\right)+\frac{g}{2} \hat{\rho}^{2}\right] .
$$

We next split the operators around the classical hydrodynamical solutions, specifically we introduce the density fluctuations $\hat{\rho}=\rho_{0}+\delta \hat{\varrho}$ and phase fluctuations $\hat{\theta}=$ $\varphi+\hat{\varphi}$.

The classical phase can be interpreted as the velocity potential by means of the equation $v=\hbar \partial_{z} \varphi / \mathrm{m}$. In a simple case where only one wall is moving we have that the classical hydrodynamic equations

$$
\begin{gathered}
\frac{\partial \rho}{\partial t}+\frac{\partial}{\partial z}(\rho v)=0, \\
\frac{\partial v}{\partial t}+v \frac{\partial v}{\partial z}=-\frac{g}{m} \frac{\partial \rho}{\partial z},
\end{gathered}
$$

have a solution given by

$$
\rho_{0}(t)=\frac{N}{L(t)}, \quad v(z, t)=z \frac{\dot{L}(t)}{L(t)} .
$$

In this case we find that the velocity depends on the position and matches the velocity of the moving wall at the boundary, namely that $v(z=0)=0$ and $v[z=L(t)]=$ $\dot{L}(t)$. This solution is obtained in the long-wavelength limit and neglecting the acceleration of the walls [169]. A similar solution can be obtained also in the case of both walls moving, with Neumann boundary conditions at each wall.

By integrating the velocity we obtain the classical phase field $\varphi$, which we next use to linearize the Hamiltonian (C78). We thus obtain the model

$$
\begin{aligned}
\hat{H}(t)= & \int_{0}^{L(t)} d z\left[\frac{\hbar^{2} \rho_{0}(z, t)}{2 m}\left(\partial_{z} \hat{\varphi}\right)^{2}+\frac{g}{2} \delta \hat{\varrho}^{2}\right. \\
& \left.+\frac{\hbar \dot{L}(t) z}{2 L(t)}\left[\delta \hat{\varrho}\left(\partial_{z} \hat{\varphi}\right)+\left(\partial_{z} \hat{\varphi}\right) \delta \hat{\varrho}\right]\right] .
\end{aligned}
$$

If the evolution is slow (adiabatic), a lattice model with the fixed number of sites can serve as a good approximation to the discrete-value representation of the continuous system. Thus, in the following we neglect the second crosscoupling term between phases and densities, so to model a quasistatic case where the GP profile gets compressed very slowly. Then, it is also illustrative to observe explicitly how this process works in an infinitesimal stepwise fashion. The infinitesimal length change is

$$
L \rightarrow L_{\epsilon}=(1+\epsilon) L,
$$

and, correspondingly, a homogeneous GP profile $\rho_{0}$ changes to $\rho_{0}(\epsilon)=(1+\epsilon)^{-1} \rho_{0}$. Then, the Hamiltonian after the size change reads

$$
\hat{H}_{\epsilon}=\int_{0}^{L_{\epsilon}} d z\left[\frac{\hbar^{2} \rho_{0}}{2 m(1+\epsilon)}\left(\partial_{z} \hat{\varphi}\right)^{2}+\frac{g}{2} \delta \hat{\varrho}^{2}\right],
$$

which is Eq. (C82) without the last term. Thus we observe that if a GP profiles changes slowly in length then the phonons are described by a similar Hamiltonian, only with modified couplings. Note that here we did not consider explicitly the phase-locking term $\hat{H}_{J}$, however, since it 
has a linear dependence on the density, it does not change while changing the total length. In the main text instead, we wrote down the full Hamiltonian with the additional (unmodified) phase-locking term, which is also what we considered in our simulations.

In the lattice model, we perform a similar procedure, but work fully in real space, this time with the Hamiltonian as a functional of both mean-field density and the small-distance cutoff. Starting from the discretized Hamiltonian $\hat{H}_{N}\left[\rho_{0}, \Delta z\right]$ of a single condensate with $N$ pixels, length $L=N \Delta z$, and density $\rho_{0}$, we perform at each step a small length change $L \mapsto L_{\epsilon}=N \Delta \zeta$, corresponding to a renormalization

$$
\begin{aligned}
H_{N}\left[\rho_{0}, \Delta z\right] & \mapsto H_{N}\left[\rho_{0}(\epsilon), \Delta \zeta\right] \\
& =H_{\rho \rho}(1+\epsilon) \oplus H_{\phi \phi} /(1+\epsilon)^{2},
\end{aligned}
$$

where we use that $\rho_{0}(\epsilon)=\rho_{0} /(1+\epsilon)$ and $\Delta \zeta=(1+$ $\epsilon) \Delta z$. Thus, we see that we are implementing a discretized version of the Hamiltonian (C84).

Then, in order to complete the full length change $\Delta L$ in a time $t_{\text {comp }}=N_{t} \Delta t$, where $\Delta t$ is a small time interval and $N_{t}$ is the total number of Trotter steps, at each discrete time step we perform an inifinitesimal length change, such that

$$
\epsilon=\Delta L / N_{t}
$$

What we get is the state of the phonons after compressing by a finite amount. This assumes that the phonons always see a quasistatic background metric, which is their dynamical time scales are much faster than how we compress the condensate. We observe that a sufficiently slow compression will not mix much between the modes and there will be thermal squeezing of the phonons. We also see that the energy will in fact change. This is expected, since we are performing work on the system by compressing it, which means it should increase in energy. The compression protocol is therefore our main way to realize a piston, where one may actively perform and extract work on a condensate by changing its length, and therefore its energy density and effective temperature.

\section{a. Renormalizing the cutoff during compression QTP}

Let us now discuss a technical detail arising in the compression and expansion QTP (see Fig. 17). An implicit difference between the initial and final Hamiltonians of a compression and expansion step is that the continuous field theory should be defined in the time-dependent line $[0, L(t)]$. In principle, we can also make a change of the integration variable $z \mapsto \zeta=z L(0) / L(t)$, such that the theory is defined with a constant length. However, a subtle issue arises: the field commutation relations $\left[\delta \hat{\varrho}(z), \hat{\varphi}\left(z^{\prime}\right)\right]=i \delta\left(z-z^{\prime}\right)$ depend on the coordinate $z$; thus a rescaling of the coordinate must be compensated by a corresponding rescaling of the density fluctuation field, in order to maintain the correct commutation relations. Then, calling $\lambda(t)=L(0) / L(t)$ we define the transformation

$$
\begin{aligned}
\zeta & =\lambda(t) z, \\
\delta \hat{v} & =\delta \hat{\varrho} / \lambda(t),
\end{aligned}
$$

such that the Hamiltonian (C82) becomes

$$
\hat{H}=\int_{0}^{L(0)} d \zeta\left[\frac{\hbar^{2} \rho_{0}(z, 0) \lambda^{2}(t)}{2 m}\left(\partial_{\zeta} \hat{\varphi}\right)^{2}+\frac{g}{2} \lambda(t) \delta \hat{v}^{2}\right],
$$

which effectively amounts to a renormalization of the line differential as

$$
d z \mapsto d \zeta=\lambda(t) d z
$$

at the same time ensuring that the fields satisfy the correct commutation relations:

$$
\begin{aligned}
{\left[\delta \hat{\varrho}(\zeta), \hat{\varphi}\left(\zeta^{\prime}\right)\right]=} & i \delta\left(\zeta-\zeta^{\prime}\right) / \lambda(t) \\
& \Rightarrow\left[\delta \hat{v}(\zeta), \hat{\varphi}\left(\zeta^{\prime}\right)\right]=i \delta\left(\zeta-\zeta^{\prime}\right)
\end{aligned}
$$

Note that by making this field transformation, the full Hamiltonian (C82) is transformed in such a way that the time derivatives of its parameters disappear. Therefore, the Hamiltonian (C88) can be approximated by a lattice model without restrictions on the rate of change of parameters, i.e., no assumption about adiabaticity is required anymore. However, one has still to be careful with defining correctly the new rescaled density-fluctuation field in the discretized model.

A similar issue arises also working directly in the discretized version of Eq. (C84): by fixing the number of pixels and just rescaling the cutoff $\Delta z$ at each Trotter step we are changing its effective momentum cutoff. Concretely, if we keep the number of pixels we see that the discretization length $\Delta \zeta$ has changed according to

$$
\Delta \zeta=\frac{L_{\epsilon}}{L} \Delta z
$$

It is important to stress once more that the covariance matrices satisfy the Heisenberg constraint that depends on $\Delta z$. Thus, we begin with a covariance matrix $\Gamma$ that satisfies

$$
\Gamma+\frac{1}{\Delta z} i \Omega \geq 0,
$$

but after size change it should satisfy

$$
\Gamma+\frac{1}{\Delta \zeta} i \Omega \geq 0
$$

However, the natural way to implement the compression is, as we discussed above, to apply a symplectic transformation $G(\epsilon)=\exp \left(\Omega H_{\epsilon} / \Delta \zeta\right)$ that preserves the symplectic 
form and hence does not allow to switch between the Heisenberg cones with $\Delta z \mapsto \Delta \zeta$. The way to implement the latter switch is to multiply the condition of the second cone and find that

$$
\Gamma+\frac{1}{\Delta z} i \Omega \geq 0 \Leftrightarrow \frac{\Delta z}{\Delta \zeta} \Gamma+\frac{1}{\Delta \zeta} i \Omega \geq 0 .
$$

Hence we can now do the compression by setting

$$
\Gamma\left(t_{\text {comp }}\right)=\frac{\Delta z}{\Delta \zeta} G\left(t_{\text {comp }}\right) \Gamma(0) G^{T}\left(t_{\text {comp }}\right),
$$

where $G\left(t_{\text {comp }}\right)$ implements the Trotterized evolution from Eq. (C85). This covariance matrix will satisfy the Heisenberg relation at the target discretization length. This is not anymore just a sympletic transformation, but an affinely symplectic transformation, which preserves the symplectic form up to an overall prefactor.

\section{Achieving larger cooling in the Otto cycle}

In the main text, we have shown how to concatenate the QTPs introduced, in order to operate a refrigerator to cool down part of the system. The remaining question is then how can we optimize the transfer of energy from the system to the bath (via piston), by tuning the various parameters that we have, such as $t_{\text {merge }}, t_{\text {split }}, t_{\text {comp }}, L_{P(B, S)}$ etc. We discuss the effects and therefore the strategy of choice for some of the parameters below.

1. Initial lengths (and length ratios) of system, piston, and bath. The lengths of each machine compartment determines their heat capacity. For example, a larger piston would be able to absorb (or lose) more heat when interacting with the system (bath). The size of the bath would largely determine how strong the non-Markovian effects are, especially since wave packets are traveling ballistically in the condensate. For example, in our simulations the bath is only 3 times larger, which is a realistic figure when considering implementations. According to Fig. 6, the wave packets induced in the bath at the piston-bath interface has already traveled to the other bath edge and returned to the interface during the second cycle of piston-bath interaction, effectively making the process non-Markovian. In Fig. 18, we see a simulation where one effectively simulates a Markovian bath (and piston) by reinitializating them before every new cycle.

2. Compression ratio of piston. It is clear that the more compression the piston undergoes, the more work is injected into the refrigerator. This causes a larger effective temperature difference between piston and bath, thereby inducing a larger amount of heat flow between them, which in turn increases the capability of the piston to later absorb heat while interacting with the system. While in classical scenarios the piston stays in equilibrium while gradually increasing in temperature, our model of compression as discussed in Sec. II B is akin to squeezing, and therefore the higher the amount of compression, the further we expect the system goes out of equilibrium, which is seen in our simulations.

3. Compression ratio of bath and system. In the protocol we presented, for simplicity, the bath and system never undergo any change in length. However, if we imagine the three condensates on a chip, whenever we compress the piston, this leaves additional room for the bath to expand. Such an additional step, if undertaken, will further increase the temperature gradient and therefore facilitate heat flow.

4. Total duration when merging and splitting two systems. Suppose two systems are connected and heat flow occurs due to an effective temperature gradient. How would one design the protocol to allow a maximum amount of net heat flow? Naturally, one expects that in the long time limit, energy will be equally distributed throughout the joint system, i.e., they thermalize. However, we are interested mostly in finite time scales. Therefore, in practice, the most relevant parameters to set are the timings of merging, with respect to the lengths of the interacting systems. Moreover, the energy input during merging is non-negligible due to the relatively small sizes of each system. To overcome this, for example, one could time the protocol so that when we split the condensates again at the end, the wave packets come back to the interface and then are taken out of the system due to the change in Hamiltonian. This can be done because we know the speed of sound in the condensate, concretely, it becomes natural to set $t_{p}=L_{p} / c$, where $t_{p}$ is a relevant time scale of the piston process. This illustrates the role of information in such a process: although a lot of energy may be injected during merging, the information about this energy is preserved, and therefore it can be suitably retrieved (instead of being irreversibly lost into other degrees of freedom).

5. Further refinements when considering the GP profile of condensates. We have seen this in the case of putting two systems into heat contact. When a single condensate sits in the trap, the bulk region has a roughly uniform density, which is why one usually considers the fairly good approximation of a homogeneous $\rho_{0}$. The situation becomes more complicated when two such systems are merged: ideally, we would like the contact interface to have large atom density as well, so that heat transport is maximized. However, we saw from the simulations that this induced extremely high-momentum 

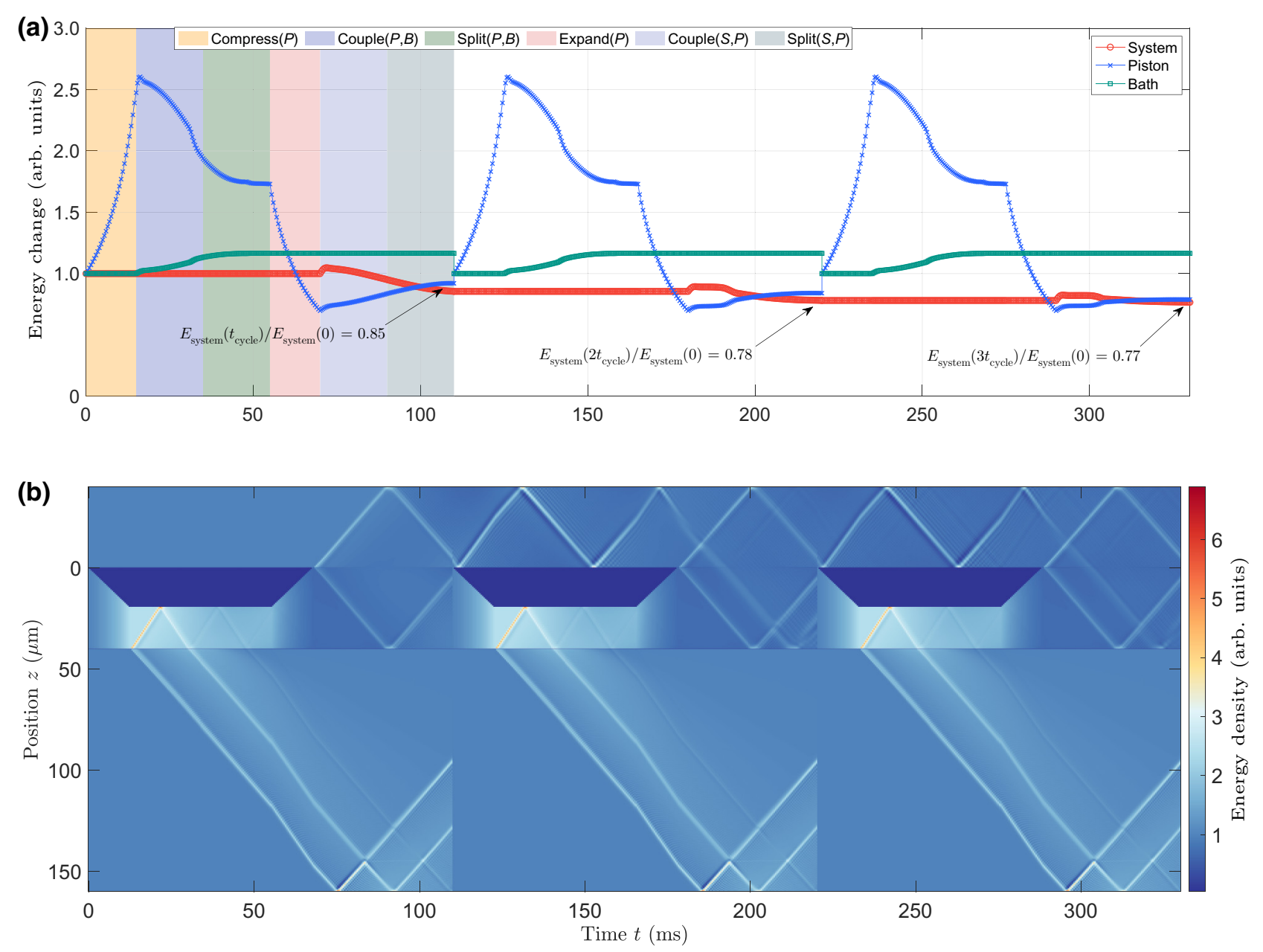

FIG. 18. In this figure, we show the Otto cycle energy changes of system, piston and bath with a different setting: $t_{\text {couple }}=20 \mathrm{~ms}$, $t_{\text {split }}=t_{\text {comp }}=20 \mathrm{~ms}, L_{\text {piston }}=L_{\text {system }}=40 \mu \mathrm{m}$, and $L_{\text {bath }}=120 \mu \mathrm{m}$. On one hand, the GP profile of the condensates at the edge drop off only slightly to about 0.8 of the peak value, which allows for more heat flow to occur between condensates during finite time, with the cost of injecting higher momentum modes into the simulation. On the other hand, after each cycle we reset the piston and the bath to its original state. This reinitialization, while challenging to perform in experiments, allows fresh thermal resources to be brought into the QFM and therefore allows us to achieve more cooling in subsequent cycles. Despite having a similar qualitative behavior as in Fig. 6, it is evident that the various specific parameters governing each of the primitives will affect the final cooling efficiency of the engine.

modes, which may cause us to observe more dispersion, and furthermore the Luttinger liquid analysis may no longer be useful in such regimes. On the other hand, having a small contact interface such as shown in the trapeze profile would imply that heat flow occurs more slowly in finite time scales.

[1] J. Goold, M. Huber, A. Riera, L. del Rio, and P. Skrzypczyk, The role of quantum information in thermodynamics - a topical review, J. Phys. A 49, 143001 (2016).

[2] G. Kurizki, E. Shahmoon, and A. Zwick, Thermal baths as quantum resources: More friends than foes?, Phys. Scr. 90, 128002 (2015).
[3] C. Gogolin and J. Eisert, Equilibration, thermalisation, and the emergence of statistical mechanics in closed quantum systems, Rep. Prog. Phys. 79, 56001 (2016).

[4] R. Kosloff, Quantum thermodynamics, Entropy 15, 2100 (2013).

[5] J. Millen and A. Xuereb, Perspective on quantum thermodynamics, New J. Phys. 18, 011002 (2016).

[6] S. Vinjanampathy and J. Anders, Quantum thermodynamics, Contemp. Phys. 57, 545 (2016).

[7] W. Niedenzu, I. Mazets, G. Kurizki, and F. Jendrzejewski, Quantized refrigerator for an atomic cloud, Quantum 3, 155 (2019).

[8] J. Roßnagel, S. Dawkins, N. Tolazzi, O. Abah, E. Lutz, F. Schmidt-Kaler, and K. Singer, A single-atom heat engine, Science 352, 325 (2016).

[9] D. von Lindenfels, O. Gräb, C. T. Schmiegelow, V. Kaushal, J. Schulz, M. T. Mitchison, J. Goold, F. Schmidt-Kaler, and U. G. Poschinger, Spin Heat Engine 
Coupled to a Harmonic-Oscillator Flywheel, Phys. Rev. Lett. 123, 080602 (2019).

[10] N. V. Horne, D. Yum, T. Dutta, P. Hänggi, J. Gong, D. Poletti, and M. Mukherjee, Single-atom energyconversion device with a quantum load, Npj Quant. Inf. 6, 37 (2020).

[11] K. Ono, S. N. Shevchenko, T. Mori, S. Moriyama, and F. Nori, Analog of a Quantum Heat Engine Using a SingleSpin Qubit, Phys. Rev. Lett. 125, 166802 (2020).

[12] J. P. Pekola, Towards quantum thermodynamics in electronic circuits, Nat. Phys. 11, 118 (2015).

[13] J. Klatzow, J. N. Becker, P. M. Ledingham, C. Weinzetl, K. T. Kaczmarek, D. J. Saunders, J. Nunn, I. A. Walmsley, R. Uzdin, and E. Poem, Experimental Demonstration of Quantum Effects in the Operation of Microscopic Heat Engines, Phys. Rev. Lett. 122, 110601 (2019).

[14] B. Rauer, Ph.D. thesis (2019).

[15] T. Schweigler, M. Gluza, M. Tajik, S. Sotiriadis, F. Cataldini, S.-C. Ji, F. S. Møller, J. Sabino, B. Rauer, J. Eisert, and J. Schmiedmayer, Decay and recurrence of nongaussian correlations in a quantum many-body system, Nat. Phys. 17, 559 (2021).

[16] J. Schmiedmayer, in Thermodynamics in the Quantum Regime, edited by F. Binder, L. A. Correa, C. Gogolin, J. Anders, and G. Adesso (Springer Nature, 2018), Chap. 34, p. 823, arXiv:1805.11539.

[17] M. Gluza, T. Schweigler, B. Rauer, C. Krumnow, J. Schmiedmayer, and J. Eisert, Quantum read-out for cold atomic quantum simulators, Comm. Phys. 3, 12 (2020).

[18] M. A. Cazalilla, Bosonizing one-dimensional cold atomic gases, J. Phys. B 37, S1 (2004).

[19] T. Giamarchi, Quantum Physics in one Dimension (Clarendon Press, Oxford, 2004).

[20] T. Schweigler, V. Kasper, S. Erne, I. E. Mazets, B. Rauer, F. Cataldini, T. Langen, T. Gasenzer, J. Berges, and J. Schmiedmayer, Experimental characterization of a quantum many-body system via higher-order correlations, Nature 545, 323 (2017).

[21] C. Mora and Y. Castin, Extension of bogoliubov theory to quasicondensates, Phys. Rev. A 67, 053615 (2003).

[22] V. N. Popov, Functional Integrals in Quantum Field Theory and Statistical Physics (Springer Science \& Business Media, Berlin, 2001), Vol. 8.

[23] V. Gritsev, A. Polkovnikov, and E. Demler, Linear response theory for a pair of coupled one-dimensional condensates of interacting atoms, Phys. Rev. B 75, 174511 (2007).

[24] R. Folman, P. Krüger, D. Cassettari, B. Hessmo, T. Maier, and J. Schmiedmayer, Controlling Cold Atoms Using Nanofabricated Surfaces: Atom Chips, Phys. Rev. Lett. 84, 4749 (2000).

[25] R. Folman, P. Krüger, J. Schmiedmayer, J. Denschlag, and C. Henkel, Microscopic Atom Optics: From Wires to an Atom Chip (Academic Press, Cambridge, MA, 2002), p. 263.

[26] J. Reichel and V. Vuletic, Atom Chips (John Wiley \& Sons, Hoboken, NY, 2011).

[27] M. Tajik, B. Rauer, T. Schweigler, F. Cataldini, J. ao Sabino, F. S. Møller, S.-C. Ji, I. E. Mazets, and
J. Schmiedmayer, Designing arbitrary one-dimensional potentials on an atom chip, Opt. Express 27, 33474 (2019).

[28] D. S. Petrov, G. V. Shlyapnikov, and J. T. M. Walraven, Regimes of Quantum Degeneracy in Trapped 1d Gases, Phys. Rev. Lett. 85, 3745 (2000).

[29] M. Lewenstein and L. You, Quantum Phase Diffusion of a Bose-Einstein Condensate, Phys. Rev. Lett. 77, 3489 (1996).

[30] J. Javanainen and M. Wilkens, Phase and Phase Diffusion of a Split Bose-Einstein Condensate, Phys. Rev. Lett. 78, 4675 (1997).

[31] A. J. Leggett and F. Sols, Comment on "Phase and Phase Diffusion of a Split Bose-Einstein Condensate", Phys. Rev. Lett. 81, 1344 (1998).

[32] J. Javanainen and M. Wilkens, Javanainen and Wilkens Reply:, Phys. Rev. Lett. 81, 1345 (1998).

[33] B. Rauer, S. Erne, T. Schweigler, F. Cataldini, M. Tajik, and J. Schmiedmayer, Recurrences in an isolated quantum many-body system, Science 359, 307 (2018).

[34] T. Schweigler, Ph.D. thesis (2019).

[35] Y. Kagan, D. L. Kovrizhin, and L. A. Maksimov, Anomalous Tunneling of Phonon Excitations Between two Bose-Einstein Condensates, Phys. Rev. Lett. 90, 130402 (2003).

[36] C. Menotti, J. R. Anglin, J. I. Cirac, and P. Zoller, Dynamic splitting of a bose-einstein condensate, Phys. Rev. A 63, 023601 (2001).

[37] M. Gring, M. Kuhnert, T. Langen, T. Kitagawa, B. Rauer, M. Schreitl, I. E. Mazets, D. A. Smith, E. Demler, and J. Schmiedmayer, Relaxation and prethermalization in an isolated quantum system, Science 337, 1318 (2012).

[38] I. Carusotto, R. Balbinot, A. Fabbri, and A. Recati, Density correlations and analog dynamical Casimir emission of Bogoliubov phonons in modulated atomic BoseEinstein condensates, Europ. Phys. J. D 56, 391 (2010).

[39] M. H. Michael, J. Schmiedmayer, and E. Demler, From the moving piston to the dynamical Casimir effect: Explorations with shaken condensates, Phys. Rev. A 99, 053615 (2019).

[40] Y.-Y. Chen, G. Watanabe, Y.-C. Yu, X.-W. Guan, and A. del Campo, An interaction-driven many-particle quantum heat engine and its universal behavior, Npj Quant. Inf. 5, 1 (2019).

[41] J. Jaramillo, M. Beau, and A. del Campo, Quantum supremacy of many-particle thermal machines, New J. Phys. 18, 075019 (2016).

[42] M. A. Cazalilla, R. Citro, T. Giamarchi, E. Orignac, and M. Rigol, One dimensional bosons: From condensed matter systems to ultracold gases, Rev. Mod. Phys. 83, 1405 (2011).

[43] T. Langen, R. Geiger, M. Kuhnert, B. Rauer, and J. Schmiedmayer, Local emergence of thermal correlations in an isolated quantum many-body system, Nat. Phys. 9, 640 (2013).

[44] T. Langen, S. Erne, R. Geiger, B. Rauer, T. Schweigler, M. Kuhnert, W. Rohringer, I. E. Mazets, T. Gasenzer, and J. Schmiedmayer, Experimental observation of a generalized Gibbs ensemble, Science 348, 207 (2015).

[45] B. Yang, Y.-Y. Chen, Y.-G. Zheng, H. Sun, H.-N. Dai, X.-W. Guan, Z.-S. Yuan, and J.-W. Pan, Quantum 
Criticality and the Tomonaga-Luttinger Liquid in OneDimensional Bose Gases, Phys. Rev. Lett. 119, 165701 (2017).

[46] R. Grimm and Y. B. Ovchinnikov, Optical dipole traps for neutral atoms, arXiv:9902072v1 (1987).

[47] M. Aidelsburger, J. L. Ville, R. Saint-Jalm, S. Nascimbène, J. Dalibard, and J. Beugnon, Relaxation Dynamics in the Merging of $n$ Independent Condensates, Phys. Rev. Lett. 119, 190403 (2017).

[48] L.-C. Ha, L. W. Clark, C. V. Parker, B. M. Anderson, and C. Chin, Roton-Maxon Excitation Spectrum of Bose Condensates in a Shaken Optical Lattice, Phys. Rev. Lett. 114, 055301 (2015).

[49] P. Zupancic, P. M. Preiss, R. Ma, A. Lukin, M. E. Tai, M. Rispoli, R. Islam, and M. Greiner, Ultra-precise holographic beam shaping for microscopic quantum control, Opt. Express 24, 13881 (2016).

[50] S. Eckel, A. Kumar, T. Jacobson, I. B. Spielman, and G. K. Campbell, A Rapidly Expanding Bose-Einstein Condensate: An Expanding Universe in the lab, Phys. Rev. X 8, 021021 (2018).

[51] K. Henderson, C. Ryu, C. MacCormick, and M. G. Boshier, Experimental demonstration of painting arbitrary and dynamic potentials for Bose-Einstein condensates, New J. Phys. 11, 043030 (2009).

[52] L. Amico et al., Roadmap on atomtronics, arXiv:2008. 04439 [cond-mat.quant-gas] (2020).

[53] W. Rohringer, D. Fischer, F. Steiner, I. E. Mazets, J. Schmiedmayer, and M. Trupke, Non-equilibrium scale invariance and shortcuts to adiabaticity in a onedimensional Bose gas, Sci. Rep. 5, 9820 (2015).

[54] V. Gritsev, P. Barmettler, and E. Demler, Scaling approach to quantum non-equilibrium dynamics of many-body systems, New J. Phys. 12, 113005 (2010).

[55] Y.-H. Wang, A. Kumar, F. Jendrzejewski, R. M. Wilson, M. Edwards, S. Eckel, G. K. Campbell, and C. W. Clark, Resonant wavepackets and shock waves in an atomtronic SQUID, New J. Phys. 17, 125012 (2015).

[56] C. Booker, B. Buča, and D. Jaksch, Non-stationarity and dissipative time crystals: Spectral properties and finitesize effects, New J. Phys. 22, 085007 (2020).

[57] S. Eckel, J. G. Lee, F. Jendrzejewski, N. Murray, C. W. Clark, C. J. Lobb, W. D. Phillips, M. Edwards, and G. K. Campbell, Hysteresis in a quantized superfluid 'atomtronic' circuit, Nature 506, 200 (2014).

[58] M. Schemmer, A. Johnson, and I. Bouchoule, Monitoring squeezed collective modes of a one-dimensional Bose gas after an interaction quench using density-ripple analysis, Phys. Rev. A 98, 043604 (2018).

[59] B. Fang, A. Johnson, T. Roscilde, and I. Bouchoule, Momentum-Space Correlations of a One-Dimensional Bose gas, Phys. Rev. Lett. 116, 050402 (2016).

[60] J. Armijo, T. Jacqmin, K. V. Kheruntsyan, and I. Bouchoule, Probing Three-Body Correlations in a Quantum Gas Using the Measurement of the Third Moment of Density Fluctuations, Phys. Rev. Lett. 105, 230402 (2010).

[61] J. Esteve, J.-B. Trebbia, T. Schumm, A. Aspect, C. I. Westbrook, and I. Bouchoule, Observations of Density Fluctuations in an Elongated Bose Gas: Ideal Gas and
Quasicondensate Regimes, Phys. Rev. Lett. 96, 130403 (2006).

[62] T. Jacqmin, J. Armijo, T. Berrada, K. V. Kheruntsyan, and I. Bouchoule, Sub-Poissonian Fluctuations in a $1 \mathrm{~d}$ Bose Gas: From the Quantum Quasicondensate to the Strongly Interacting Regime, Phys. Rev. Lett. 106, 230405 (2011).

[63] A. Imambekov, I. E. Mazets, D. S. Petrov, V. Gritsev, S. Manz, S. Hofferberth, T. Schumm, E. Demler, and J. Schmiedmayer, Density ripples in expanding lowdimensional gases as a probe of correlations, Phys. Rev. A 80, 033604 (2009).

[64] S. Manz, Ph.D. thesis, Vienna University of Technology (2011).

[65] T. Schumm, S. Hofferberth, L. M. Andersson, S. Wildermuth, S. Groth, I. Bar-Joseph, J. Schmiedmayer, and P. Kruger, Matter-wave interferometry in a double well on an atom chip, Nat. Phys. 1, 57 (2005).

[66] Y. D. van Nieuwkerk, J. Schmiedmayer, and F. H. L. Essler, Projective phase measurements in one-dimensional Bose gases, Scipost Phys. 5, 046 (2018).

[67] S. Hofferberth, I. Lesanovsky, T. Schumm, A. Imambekov, V. Gritsev, E. Demler, and J. Schmiedmayer, Probing quantum and thermal noise in an interacting many-body system, Nat. Phys. 4, 489 (2008).

[68] K. B. Davis, M.-O. Mewes, and W. Ketterle, An analytical model for evaporative cooling of atoms, Appl. Phys. B 60, 155 (1995).

[69] I. E. Mazets, T. Schumm, and J. Schmiedmayer, Breakdown of Integrability in a Quasi-1D Ultracold Bosonic Gas, Phys. Rev. Lett. 100, 210403 (2008).

[70] S. Tan, M. Pustilnik, and L. I. Glazman, Relaxation of a High-Energy Quasiparticle in a One-Dimensional Bose Gas, Phys. Rev. Lett. 105, 090404 (2010).

[71] A. Andreev, The hydrodynamics of two-and onedimensional liquids, JETP 51, 1038 (1980).

[72] M. Buchhold and S. Diehl, Kinetic theory for interacting Luttinger liquids, Europ. Phys. J. D 69, 1 (2015).

[73] B. Rauer, P. Grišins, I. E. Mazets, T. Schweigler, W. Rohringer, R. Geiger, T. Langen, and J. Schmiedmayer, Cooling of a One-Dimensional Bose Gas, Phys. Rev. Lett. 116, 030402 (2016).

[74] P. Grišins, B. Rauer, T. Langen, J. Schmiedmayer, and I. E. Mazets, Degenerate Bose gases with uniform loss, Phys. Rev. A 93, 033634 (2016).

[75] X. Busch, I. Carusotto, and R. Parentani, Spectrum and entanglement of phonons in quantum fluids of light, Phys. Rev. A 89, 043819 (2014).

[76] Y. Japha, S. Choi, K. Burnett, and Y. B. Band, Coherent Output, Stimulated Quantum Evaporation, and Pair Breaking in a Trapped Atomic Bose Gas, Phys. Rev. Lett. 82, 1079 (1999).

[77] Note also that experiments with nuclear magnetic resonance have been performed realizing a quantum Otto heat engine operating under a reservoir at effective negative temperatures [170].

[78] G.-B. Jo, Y. Shin, S. Will, T. A. Pasquini, M. Saba, W. Ketterle, D. E. Pritchard, M. Vengalattore, and M. Prentiss, Long Phase Coherence Time and Number 
Squeezing of two Bose-Einstein Condensates on an Atom Chip, Phys. Rev. Lett. 98, 030407 (2007).

[79] J. Estève, C. Gross, a. Weller, S. Giovanazzi, and M. $\mathrm{K}$. Oberthaler, Squeezing and entanglement in a BoseEinstein condensate, Nature 455, 1216 (2008).

[80] T. Berrada, S. V. Frank, R. Bücker, T. Schumm, J.-F. Schaff, and J. Schmiedmayer, Integrated MachZehnder interferometer for Bose-Einstein condensates, Nat. Comm. 4, 2077 (2013).

[81] L. del Rio, J. Aberg, R. Renner, O. Dahlsten, and V. Vedral, The thermodynamic meaning of negative entropy, Nature 474, 61 (2011).

[82] D. Jennings and T. Rudolph, Entanglement and the thermodynamic arrow of time, Phys. Rev. E 81, 061130 (2010).

[83] M. H. Partovi, Entanglement versus stosszahlansatz: Disappearance of the thermodynamic arrow in a highcorrelation environment, Phys. Rev. E 77, 021110 (2008).

[84] S. Jevtic, D. Jennings, and T. Rudolph, Maximally and Minimally Correlated States Attainable within a Closed Evolving System, Phys. Rev. Lett. 108, 110403 (2012).

[85] L. del Rio, A. Hutter, R. Renner, and S. Wehner, Relative thermalization, Phys. Rev. E 94, 022104 (2016).

[86] K. Micadei, J. P. Peterson, A. M. Souza, R. S. Sarthour, I. S. Oliveira, G. T. Landi, T. B. Batalhão, R. M. Serra, and E. Lutz, Reversing the direction of heat flow using quantum correlations, Nat. Comm. 10, 2456 (2019).

[87] D. Husmann, M. Lebrat, S. Häusler, J.-P. Brantut, L. Corman, and T. Esslinger, Breakdown of the WiedemannFranz law in a unitary Fermi gas, Proc. Natl. Ac. Sc. 115, 8563 (2018).

[88] H.-P. Breuer, E.-M. Laine, J. Piilo, and B. Vacchini, Colloquium: Non-Markovian dynamics in open quantum systems, Rev. Mod. Phys. 88, 021002 (2016).

[89] A. Rivas, S. F. Huelga, and M. B. Plenio, Quantum nonMarkovianity: Characterization, quantification and detection, Rep. Prog. Phys. 77, 094001 (2014).

[90] P. Boes, R. Gallego, N. H. Ng, J. Eisert, and H. Wilming, By-passing fluctuation theorems, Quantum 4, 231 (2020).

[91] J.-P. Brantut, C. Grenier, J. Meineke, D. Stadler, S. Krinner, C. Kollath, T. Esslinger, and A. Georges, A thermoelectric heat engine with ultracold atoms, Science 342, 713 (2013).

[92] J. Anders and A. Winter, Entanglement and separability of quantum harmonic oscillator systems at finite temperature, arXiv:0705.3026 (2007).

[93] J. Anders, Thermal state entanglement in harmonic lattices, Phys. Rev. A 77, 062102 (2008).

[94] P. Calabrese and J. Cardy, Entanglement entropy and quantum field theory, J. Stat. Mech. 2004, P06002 (2004).

[95] M. M. Wolf, J. Eisert, T. S. Cubitt, and J. I. Cirac, Assessing non-Markovian Quantum Dynamics, Phys. Rev. Lett. 101, 150402 (2008).

[96] A. Rivas, S. F. Huelga, and M. B. Plenio, Entanglement and Non-Markovianity of Quantum Evolutions, Phys. Rev. Lett. 105, 050403 (2010).

[97] G. Gour, M. P. Müller, V. Narasimhachar, R. W. Spekkens, and N. Y. Halpern, The resource theory of informational nonequilibrium in thermodynamics, Phys. Rep. 583, 1 (2015).
[98] S. Vinjanampathy and J. Anders, Quantum thermodynamics, Contemp. Phys. 57, 545 (2016).

[99] R. Bistritzer and E. Altman, Intrinsic dephasing in onedimensional ultracold atom interferometers, Proc. Natl. Ac. Sc. 104, 9955 (2007).

[100] T. Kitagawa, A. Imambekov, J. Schmiedmayer, and E. Demler, The dynamics and prethermalization of onedimensional quantum systems probed through the full distributions of quantum noise, New J. Phys. 13, 073018 (2011).

[101] R. Geiger, T. Langen, I. E. Mazets, and J. Schmiedmayer, Local relaxation and light-cone-like propagation of correlations in a trapped one-dimensional Bose gas, New J. Phys. 16, 053034 (2014).

[102] M. Pezzutto, M. Paternostro, and Y. Omar, Implications of non-Markovian quantum dynamics for the Landauer bound, New J. Phys. 18, 123018 (2016).

[103] P. P. Hofer, M. Perarnau-Llobet, L. D. M. Miranda, G. Haack, R. Silva, J. B. Brask, and N. Brunner, Markovian master equations for quantum thermal machines: Local versus global approach, New J. Phys. 19, 123037 (2017).

[104] J. O. González, L. A. Correa, G. Nocerino, J. P. Palao, D. Alonso, and G. Adesso, Testing the validity of the local and global GKLS master equations on an exactly solvable model, Open Sys. Inf. Dyn. 24, 1740010 (2017).

[105] R. Uzdin, A. Levy, and R. Kosloff, Quantum heat machines equivalence, work extraction beyond markovianity, and strong coupling via heat exchangers, Entropy 18, 124 (2016).

[106] S. Groeblacher, A. Trubarov, N. Prigge, M. Aspelmeyer, and J. Eisert, Observation of non-Markovian micromechanical Brownian motion, Nat. Comm. 6, 7606 (2015).

[107] F. Brandao, M. Horodecki, N. Ng, J. Oppenheim, and S. Wehner, The second laws of quantum thermodynamics, Proc. Natl. Ac. Sc. 112, 3275 (2015).

[108] Á. M. Alhambra, L. Masanes, J. Oppenheim, and C. Perry, Fluctuating Work: From Quantum Thermodynamical Identities to a Second Law Equality, Phys. Rev. X 6, 041017 (2016).

[109] A. Serafini, M. Lostaglio, S. Longden, U. ShackerleyBennett, C.-Y. Hsieh, and G. Adesso, Gaussian Thermal Operations and the Limits of Algorithmic Cooling, Phys. Rev. Lett. 124, 010602 (2020).

[110] N. Y. Halpern and D. T. Limmer, Fundamental limitations on photoisomerization from thermodynamic resource theories, Phys. Rev. A 101, 042116 (2020).

[111] F. Clivaz, R. Silva, G. Haack, J. B. Brask, N. Brunner, and M. Huber, Unifying Paradigms of Quantum Refrigeration: A Universal and Attainable Bound on Cooling, Phys. Rev. Lett. 123, 170605 (2019).

[112] F. Clivaz, R. Silva, G. Haack, J. B. Brask, N. Brunner, and M. Huber, Unifying paradigms of quantum refrigeration: Fundamental limits of cooling and associated work costs, Phys. Rev. E 100, 042130 (2019).

[113] M. P. Woods, N. H. Y. Ng, and S. Wehner, The maximum efficiency of nano heat engines depends on more than temperature, Quantum 3, 177 (2019). 
[114] H. Wilming and R. Gallego, Third law of Thermodynamics as a Single Inequality, Phys. Rev. X 7, 041033 (2017).

[115] L. Masanes and J. Oppenheim, A general derivation and quantification of the third law of thermodynamics, Nat. Comm. 8, 14538 (2017).

[116] W. Niedenzu, M. Huber, and E. Boukobza, Concepts of work in autonomous quantum heat engines, Quantum 3, 195 (2019).

[117] C. Jarzynski, Nonequilibrium Equality for Free Energy Differences, Phys. Rev. Lett. 78, 2690 (1997).

[118] M. Horodecki and J. Oppenheim, Fundamental limitations for quantum and nano thermodynamics, Nat. Comm. 4, 2059 (2013).

[119] F. G. S. L. Brandao, M. Horodecki, J. Oppenheim, J. M. Renes, and R. W. Spekkens, Resource Theory of Quantum States out of Thermal Equilibrium, Phys. Rev. Lett. 111, 250404 (2013).

[120] C. T. Chubb, M. Tomamichel, and K. Korzekwa, Beyond the thermodynamic limit: Finite-size corrections to state interconversion rates, Quantum 2, 108 (2018).

[121] R. Gallego, J. Eisert, and H. Wilming, Thermodynamic work from operational principles, New J. Phys. 18, 103017 (2016).

[122] L. del Rio, L. Kraemer, and R. Renner, Resource theories of knowledge, arXiv:1511.08818 (2015).

[123] M. Perarnau-Llobet, K. V. Hovhannisyan, M. Huber, P. Skrzypczyk, N. Brunner, and A. Acin, Extractable Work from Correlations, Phys. Rev. X 5, 041011 (2015).

[124] N. Brunner, M. Huber, N. Linden, S. Popescu, R. Silva, and P. Skrzypczyk, Entanglement enhances cooling in microscopic quantum refrigerators, Phys. Rev. E 89, 032115 (2014).

[125] N. H. Y. Ng, M. P. Woods, and S. Wehner, Surpassing the carnot efficiency by extracting imperfect work, New J. Phys. 19, 113005 (2017).

[126] I. Kukuljan, S. Sotiriadis, and G. Takacs, Correlation Functions of the Quantum sine-Gordon Model in and out of Equilibrium, Phys. Rev. Lett. 121, 110402 (2018).

[127] V. Narasimhachar, S. Assad, F. C. Binder, J. Thompson, B. Yadin, and M. Gu, Thermodynamic resources in continuous-variable quantum systems, arXiv:1909.07364 (2019).

[128] S. Coleman, Quantum Sine-Gordon equation as the massive Thirring model, Phys. Rev. D 11, 2088 (1975).

[129] S. Mandelstam, Soliton operators for the quantized SineGordon equation, Phys. Rev. D 11, 3026 (1975).

[130] W. E. Thirring, A soluble relativistic field theory, Ann. Phys. 3, 91 (1958).

[131] L. D. Faddeev and V. E. Korepin, Quantum theory of solitons, Phys. Rep. 42, 1 (1978).

[132] T. V. Zache, T. Schweigler, S. Erne, J. Schmiedmayer, and J. Berges, Extracting the Field Theory Description of a Quantum Many-Body System from Experimental Data, Phys. Rev. X 10, 11020 (2020).

[133] A. D. Cronin, J. Schmiedmayer, and D. E. Pritchard, Optics and interferometry with atoms and molecules, Rev. Mod. Phys. 81, 1051 (2009).

[134] J. Grond, J. Schmiedmayer, and U. Hohenester, Optimizing number squeezing when splitting a mesoscopic condensate, Phys. Rev. A 79, 021603 (2009).
[135] J. Werschnik and E. Gross, Quantum optimal control theory, J. Phys. B 40, R175 (2007).

[136] T. Caneva, M. Murphy, T. Calarco, R. Fazio, S. Montangero, V. Giovannetti, and G. E. Santoro, Optimal Control at the Quantum Speed Limit, Phys. Rev. Lett. 103, 240501 (2009).

[137] P. Doria, T. Calarco, and S. Montangero, Optimal Control Technique for Many-Body Quantum Dynamics, Phys. Rev. Lett. 106, 190501 (2011).

[138] C. P. Koch, Controlling open quantum systems: Tools, achievements, and limitations, J. Phys. 28, 213001 (2016).

[139] S. van Frank, M. Bonneau, J. Schmiedmayer, S. Hild, C. Gross, M. Cheneau, I. Bloch, T. Pichler, A. Negretti, T. Calarco, and S. Montangero, Optimal control of complex atomic quantum systems, Sci. Rep. 6, 34187 (2016).

[140] P. Hauke, M. Lewenstein, and A. Eckardt, Tomography of Band Insulators from Quench Dynamics, Phys. Rev. Lett. 113, 045303 (2014).

[141] J.-F. Schaff, T. Langen, and J. Schmiedmayer, Interferometry with atoms, Rivista del Nuovo Cimento Della Societa Italiana di Fisica 37, 509 (2014).

[142] J. Eisert, D. Hangleiter, N. Walk, I. Roth, D. Markham, R. Parekh, U. Chabaud, and E. Kashefi, Quantum certification and benchmarking, Nat. Rev. Phys. 2, 382 (2020).

[143] B. Yang, H. Sun, C.-J. Huang, H.-Y. Wang, Y. Deng, H.N. Dai, Z.-S. Yuan, and J.-W. Pan, Cooling and entangling ultracold atoms in optical lattices, Science (2020).

[144] S. Wildermuth, S. Hofferberth, I. Lesanovsky, E. Haller, L. M. Andersson, S. Groth, I. Bar-Joseph, P. Krüger, and J. Schmiedmayer, Microscopic magnetic-field imaging, Nature 435, 440 (2005).

[145] S. Aigner, L. Della Pietra, Y. Japha, O. Entin-Wohlman, T. David, R. Salem, R. Folman, and J. Schmiedmayer, Longrange order in electronic transport through disordered metal films, Science 319, 1226 (2008).

[146] M. Andrews, M.-O. Mewes, N. Van Druten, D. Durfee, D. Kurn, and W. Ketterle, Direct, nondestructive observation of a bose condensate, Science 273, 84 (1996).

[147] M. Saba, T. Pasquini, C. Sanner, Y. Shin, W. Ketterle, and D. Pritchard, Light scattering to determine the relative phase of two Bose-Einstein condensates, Science 307, 1945 (2005).

[148] D. V. Freilich, D. M. Bianchi, A. M. Kaufman, T. K. Langin, and D. S. Hall, Real-time dynamics of single vortex lines and vortex dipoles in a Bose-Einstein condensate, Science 329, 1182 (2010).

[149] M. J. H. Ku, B. Mukherjee, T. Yefsah, and M. W. Zwierlein, Cascade of Solitonic Excitations in a Superfluid Fermi Gas: From Planar Solitons to Vortex Rings and Lines, Phys. Rev. Lett. 116, 045304 (2016).

[150] S. Serafini, L. Galantucci, E. Iseni, T. Bienaimé, R. N. Bisset, C. F. Barenghi, F. Dalfovo, G. Lamporesi, and G. Ferrari, Vortex Reconnections and Rebounds in Trapped Atomic Bose-Einstein Condensates, Phys. Rev. X 7, 021031 (2017).

[151] E. M. Seroka, A. V. Curiel, D. Trypogeorgos, N. Lundblad, and I. B. Spielman, Repeated measurements with minimally destructive partial-transfer absorption imaging, Opt. Express 27, 36611 (2019).

[152] R. Bücker, A. Perrin, S. Manz, T. Betz, C. Koller, T. Plisson, J. Rottmann, T. Schumm, and J. Schmiedmayer, 
Single-particle-sensitive imaging of freely propagating ultracold atoms, New J. Phys. 11, 103039 (2009).

[153] A. Bergschneider, V. M. Klinkhamer, J. H. Becher, R. Klemt, G. Zürn, P. M. Preiss, and S. Jochim, Spin-resolved single-atom imaging of $6 \mathrm{Li}$ in free space, Phys. Rev. A 97, 063613 (2018).

[154] J.-Y. Choi, S. Hild, J. Zeiher, P. Schauß, A. Rubio-Abadal, T. Yefsah, V. Khemani, D. A. Huse, I. Bloch, and C. Gross, Exploring the many-body localization transition in two dimensions, Science 352, 1547 (2016).

[155] T. Fukuhara, A. Kantian, M. Endres, M. Cheneau, P. Schauss, S. Hild, Bellem, U. Schollwöck, T. Giamarchi, C. Gross, I. Bloch, and S. Kuhr, Quantum dynamics of a single, mobile spin impurity, Nat. Phys. 9, 235 (2013).

[156] Q. Bouton, J. Nettersheim, S. Burgardt, D. Adam, E. Lutz, and A. Widera, A quantum heat engine driven by atomic collisions, Nat. Comm. 12, 2063 (2021).

[157] L. Arrachea, E. R. Mucciolo, C. Chamon, and R. B. Capaz, Microscopic model of a phononic refrigerator, Phys. Rev. B 86, 125424 (2012).

[158] C. Chamon, E. R. Mucciolo, L. Arrachea, and R. B. Capaz, Heat Pumping in Nanomechanical Systems, Phys. Rev. Lett. 106, 135504 (2011).

[159] M. Pigneur, T. Berrada, M. Bonneau, T. Schumm, E. Demler, and J. Schmiedmayer, Relaxation to a Phase-Locked Equilibrium State in a One-Dimensional Bosonic Josephson Junction, Phys. Rev. Lett. 120, 173601 (2018).

[160] D. Gelbwaser-Klimovsky, A. Bylinskii, D. Gangloff, R. Islam, A. Aspuru-Guzik, and V. Vuletic, Single-Atom
Heat Machines Enabled by Energy Quantization, Phys. Rev. Lett. 120, 170601 (2018).

[161] C. Weedbrook, S. Pirandola, R. Garcia-Patron, N. J. Cerf, T. C. Ralph, J. H. Shapiro, and S. Lloyd, Gaussian quantum information, Rev. Mod. Phys. 84, 621 (2012).

[162] J. Eisert and M. B. Plenio, Introduction to the basics of entanglement theory in continuous-variable systems, Int. J. Quant. Inf. 1, 479 (2003).

[163] This means that the density matrix has no zero eigenvalue.

[164] J. Javanainen, Phonon approach to an array of traps containing Bose-Einstein condensates, Phys. Rev. A 60, 4902 (1999).

[165] L. Salasnich, A. Parola, and L. Reatto, Effective wave equations for the dynamics of cigar-shaped and diskshaped bose condensates, Phys. Rev. A 65, 043614 (2002).

[166] M. Gluza, J. Eisert, and T. Farrelly, Equilibration towards generalized Gibbs ensembles in non-interacting theories, SciPost Phys. 7, 38 (2019).

[167] M. Pigneur and J. Schmiedmayer, Analytical pendulum model for a bosonic Josephson junction, Phys. Rev. A 98, 063632 (2018).

[168] N. K. Whitlock and I. Bouchoule, Relative phase fluctuations of two coupled one-dimensional condensates, Phys. Rev. A 68, 053609 (2003).

[169] F. Dalfovo, S. Giorgini, L. P. Pitaevskii, and S. Stringari, Theory of Bose-Einstein condensation in trapped gases, Rev. Mod. Phys. 71, 463 (1999).

[170] R. J. de Assis, T. M. de Mendonça, C. J. Villas-Boas, A. M. de Souza, R. S. Sarthour, I. S. Oliveira, and N. G. de Almeida, Efficiency of a Quantum Otto Heat Engine Operating under a Reservoir at Effective Negative Temperatures, Phys. Rev. Lett. 122, 240602 (2019). 